\title{
Article
}

\section{SONS: The JCMT legacy survey of debris discs in the submillimetre}

Holland, Wayne S., Matthews, Brenda C., Kennedy, Grant M., Greaves, Jane S., Wyatt, Mark C., Booth, Mark, Bastien, Pierre, Bryden, Geoff, Butner, Harold, Chen, Christine H., Chrysostomou, Antonio, Davies, Claire L., Dent, William R. F., Di Francesco, James, Duchêne, Gaspard, Gibb, Andy G., Friberg, Per, Ivison, Rob J., Jenness, Tim, Kavelaars, JJ, Lawler, Samantha, Lestrade, JeanFrançois, Marshall, Jonathan P., Moro-Martin, Amaya, Panić, Olja, Phillips, Neil, Serjeant, Stephen, Schieven, Gerald H., Sibthorpe, Bruce, Vican, Laura, Ward-Thompson, Derek, van der Werf, Paul, White, Glenn J., Wilner, David and Zuckerman, Ben

Available at http://clok.uclan.ac.uk/21680/

Holland, Wayne S., Matthews, Brenda C., Kennedy, Grant M., Greaves, Jane S., Wyatt, Mark C., Booth, Mark, Bastien, Pierre, Bryden, Geoff, Butner, Harold et al (2017) SONS: The JCMT legacy survey of debris discs in the submillimetre. Monthly Notices of the Royal Astronomical Society, 470 (3). pp. 3606-3663. ISSN 0035-8711

It is advisable to refer to the publisher's version if you intend to cite from the work. http://dx.doi.org/10.1093/mnras/stx1378

For more information about UCLan's research in this area go to http://www.uclan.ac.uk/researchgroups/ and search for < name of research Group>.

For information about Research generally at UCLan please go to http://www.uclan.ac.uk/research/

All outputs in CLoK are protected by Intellectual Property Rights law, including Copyright law. Copyright, IPR and Moral Rights for the works on this site are retained by the individual authors and/or other copyright owners. Terms and conditions for use of this material are defined in the policies page. 


\title{
SONS: The JCMT legacy survey of debris discs in the submillimetre
}

\author{
Wayne S. Holland, ${ }^{1,2 \star}$ Brenda C. Matthews, ${ }^{3,4 \star}$ Grant M. Kennedy, ${ }^{5 \star}$ \\ Jane S. Greaves,${ }^{6} \dagger$ Mark C. Wyatt, ${ }^{5}$ Mark Booth, ${ }^{7,8}$ Pierre Bastien,,${ }^{9}$ Geoff Bryden, ${ }^{10}$ \\ Harold Butner, ${ }^{11}$ Christine H. Chen, ${ }^{12}$ Antonio Chrysostomou, ${ }^{13} \ddagger$ Claire L. Davies, ${ }^{6} \$$ \\ William R. F. Dent, ${ }^{14}$ James Di Francesco, ${ }^{3,4}$ Gaspard Duchêne, ${ }^{15,16}$ Andy G. Gibb, ${ }^{17}$ \\ Per Friberg, ${ }^{18}$ Rob J. Ivison, ${ }^{2,19}$ Tim Jenness, ${ }^{18} \|$ JJ Kavelaars, ${ }^{3,4}$ Samantha Lawler, ${ }^{3,4}$ \\ Jean-François Lestrade, ${ }^{20}$ Jonathan P. Marshall, ${ }^{21,22,23}$ Amaya Moro-Martin, ${ }^{12,24}$ \\ Olja Panić, ${ }^{\star \star \star}$ Neil Phillips, ${ }^{14}$ Stephen Serjeant, ${ }^{25}$ Gerald H. Schieven, ${ }^{3,4}$ \\ Bruce Sibthorpe ${ }^{26} \dagger \dagger$ Laura Vican, ${ }^{27}$ Derek Ward-Thompson, ${ }^{28}$ Paul van der Werf, ${ }^{29}$ \\ Glenn J. White, ${ }^{25,30}$ David Wilner ${ }^{31}$ and Ben Zuckerman ${ }^{27}$ \\ Affiliations are listed at the end of the paper
}

Accepted 2017 June 1. Received 2017 June 1; in original form 2017 April 13

\begin{abstract}
Debris discs are evidence of the ongoing destructive collisions between planetesimals, and their presence around stars also suggests that planets exist in these systems. In this paper, we present submillimetre images of the thermal emission from debris discs that formed the SCUBA-2 Observations of Nearby Stars (SONS) survey, one of seven legacy surveys undertaken on the James Clerk Maxwell Telescope between 2012 and 2015. The overall results of the survey are presented in the form of $850 \mu \mathrm{m}$ (and $450 \mu \mathrm{m}$, where possible) images and fluxes for the observed fields. Excess thermal emission, over that expected from the stellar photosphere, is detected around 49 stars out of the 100 observed fields. The discs are characterized in terms of their flux density, size (radial distribution of the dust) and derived dust properties from their spectral energy distributions. The results show discs over a range of sizes, typically 1-10 times the diameter of the Edgeworth-Kuiper Belt in our Solar system. The mass of a disc, for particles up to a few millimetres in size, is uniquely obtainable with submillimetre observations and this quantity is presented as a function of the host stars' age, showing a tentative decline in mass with age. Having doubled the number of imaged discs at submillimetre wavelengths from ground-based, single-dish telescope observations, one of the key legacy products from the SONS survey is to provide a comprehensive target list to observe at high angular resolution using submillimetre/millimetre interferometers (e.g. Atacama Large Millimeter Array, Smithsonian Millimeter Array).
\end{abstract}

Key words: circumstellar matter-submillimetre: stars.

\footnotetext{
^E-mail: wayne.holland@stfc.ac.uk (WSH); Brenda.Matthews@nrc-cnrc. gc.ca (BCM); gkennedy @ast.cam.ac.uk (GMK)

$\dagger$ Present address: School of Physics, and Astronomy, Cardiff University, 5 The Parade, Cardiff, CF24 3AA, UK.

$\ddagger$ Present address: SKA Organisation, Jodrell Bank Observatory, Lower Withington, Macclesfield, Chesire, SK11 9DL, UK.

$\S$ Present address: School of Physics, University of Exeter, Physics Building, Stocker Road, Exeter, EX4 4QL, UK.

ФPresent address: East Asian Observatory, 660 N. A 'ohōkū Place, University Park, Hilo, HI 96720, USA.

|| Present address: LSST Project Office, 950 N. Cherry Avenue, Tucson, AZ 85719, USA.

^^Present address: School of Physics, and Astronomy, E C Stoner Building, University of Leeds, Leeds, LS2 9JT, UK.
}

\section{INTRODUCTION}

Debris discs represent the longest-lived phase in the lifetime of circumstellar discs. Following the decline of the gas-rich protoplanetary phase when agglomeration processes prevail, the remnant mass of circumstellar discs is dominated by planetesimals, which undergo collisional grinding down to smaller and smaller bodies, until particles reach the blow-out size determined by the radiation pressure from the host star (e.g. Wyatt 2008; Krivov 2010). The presence of

$\dagger †$ Present address: Astrium Airbus Defence, Space, Gunnels Wood Road, Stevenage, SG1 2AS, UK. 
these unseen planetesimals can be inferred through scattered light or thermal emission from micron to millimetre-sized dust grains. The dust must be continuously replenished, by ongoing collisions between the aforementioned planetesimals, since the time-scales for dust grains to be removed from the system are significantly shorter than the ages of the stars around which they are observed (Backman \& Paresce 1993). It appears to be the case that debris discs can persist over all stages following the pre-main-sequence phase of stellar evolution (e.g. Bonsor et al. 2013), even including white dwarfs (e.g. Farihi 2016).

Observations at submillimetre/millimetre wavelengths are immensely valuable to the study of debris discs in that they trace the Rayleigh-Jeans tail of the outer cold dust in a system (Matthews et al. 2014a). For example, they probe substantially different (thermal) emission mechanisms than scattered light observations, and lower characteristic temperatures for the material than far-infrared (far-IR) data. These long wavelengths also provide an important anchor to the flux energy distribution (loosely referred to in this paper as the spectral energy distribution, or SED) in an otherwise poorly constrained wavelength range, and can indicate the presence of any (cold) disc components not detectable at shorter wavelengths. By probing the Rayleigh-Jeans tail of the spectrum, the effect of any possible bias introduced by modelling the dust temperatures from the observed data is minimized, thus allowing information to be derived on the radial distribution of the disc and the size distribution of the emitting grains (Ertel et al. 2012; Marshall et al. 2014b). The slope of the spectrum constrains the dust size distribution, providing a test of whether or not the solids in the disc are undergoing a steady-state collisional cascade. Critically, since the emission is optically thin, the dust mass for grain sizes up to $\sim 1 \mathrm{~mm}$ is uniquely determined from submillimetre data.

The disc component (in millimetre-sized grains) probed in the submillimetre is also unique from the perspective of understanding disc dynamics. These relatively large dust grains are less affected by the radiation or stellar wind pressure (Burns, Lamy \& Soter 1979) and therefore trace the location of their parent planetesimal belts more reliably than smaller grains at shorter wavelengths. Debris discs act as important pointers to planetary systems (Kóspál et al. 2009) with features in the discs having the potential to highlight the presence of planets, even in cases where the planet is as yet undetected, or would be difficult to detect by any other method, including direct imaging (e.g. Wyatt 2003, 2006). For example, the planet around $\beta$ Pictoris was predicted due to structure in the debris disc through scattered light imaging before the planet was found (Mouillet et al. 1997; Heap et al. 2000). Whilst scattered light observations are sensitive to the small grains around a given star, the bulk of the mass resides in the largest grains most detectable at submillimetre to centimetre wavelengths. These grains are most likely to be located in or near the planetesimal belts, and hence may show evidence of perturbed geometries due to resonances with long-period planets (Wyatt 2006).

The James Clerk Maxwell Telescope (JCMT) has a long history of debris disc studies (e.g. Zuckerman \& Beckin 1993), including some of the earliest imaging using the SCUBA camera (Holland et al. 1999). At the time of its decommissioning in 2005, half of the resolved images of debris discs (about a dozen in total) were due to submillimetre imaging with SCUBA (e.g. Holland et al. 1998; Greaves et al. 2005). Subsequent surveys in the mid-far-IR (e.g. using Spitzer, AKARI and Herschel) identified a large sample of discs in the solar vicinity (to a distance of $\sim 100 \mathrm{pc}$ ). For example, the Herschel DEBRIS (Disk Emission via a Bias-Free Reconnaissance in the Infrared/Submillimetre) survey observed the nearest $\sim 90$ stars in each of the spectral type groups A, F, G, K and M, obtaining a disc detection rate of 17 per cent based on 100 and $160 \mu \mathrm{m}$ results, corresponding to 77 out of a total of 446 targets detected (Matthews et al. 2014). Similarly, the Herschel DUNES (DUst around NEarby Stars) survey detected an incidence of 20 per cent for nearby Sunlike stars, probing to the photospheric level (Eiroa et al. 2013; Montesinos et al. 2016). In terms of limits to the detectable flux, Spitzer and Herschel achieved average sensitivities, expressed as fractional dust luminosities (see Section 4.4) of $\sim 10^{-5}$ and $\sim 10^{-6}$, respectively. These levels compare to $\sim 10^{-7}$ for the EdgeworthKuiper belt in our Solar system. Crucially, the surveys by Herschel spatially resolved half of the detected discs, many for the first time. Other surveys, in the near-mid IR with $A K A R I$, have probed 'warmer' debris discs ( $T \geq 150 \mathrm{~K})$, i.e. material to be found closer to the central star, with incidence rates typically 3 per cent, much lower than for the 'cooler' discs detected at longer wavelengths (Fujiwara et al. 2013).

The SCUBA-2 Observations of Nearby Stars (SONS) survey was one of the seven original legacy surveys undertaken on the JCMT between 2012 and 2015 (Chrysostomou 2010). The survey set out to target 115 known disc host stars (within $100 \mathrm{pc}$ of the Sun) searching for debris signatures in the form of dust emission at $850 \mu \mathrm{m}$. The aim of the SONS survey was to characterize these discs to the fullest extent possible by (1) providing direct dust masses that cannot be obtained from shorter wavelengths alone; (2) adding to the far-IR/submillimetre spectrum to constrain the dust size distribution; (3) using the power of a $15 \mathrm{~m}$ telescope to resolve disc structures around the nearest systems; and (4) looking for evidence of resonant clumps and other features in resolved structures that could be indicative of unseen perturbers, such as planets. This paper presents the first results from the full survey. Future work will concentrate on detailed modelling of the disc structures, further investigations into dust grain properties and size distributions, and their interpretation in terms of the relationship to possible planetary systems.

\section{SURVEY HISTORY AND TARGET SELECTION}

The original concept was for a volume-limited, unbiased survey of 500 stars, the 100 nearest in each of the spectral type groups A, F, G, K and M (Matthews et al. 2007a). First formulated in 2004, this was called the SCUBA-2 Unbiased Nearby Stars (SUNS) survey. The aforementioned extensive surveys by Spitzer and Herschel during the period 2004-2012, together with a shortfall in instrument sensitivity of approximately a factor of 2, however, meant that the greatest potential legacy lay in a revamped JCMT/SCUBA-2 survey to target a more modest number of known debris disc hosts. Discs would have to be very cold to be detectable with SCUBA-2 but below the detection threshold of, for example, the Herschel DEBRIS survey. Hence the SONS survey became targeted towards younger stars, and stars with known IR excesses, with a higher expectation of detection at a wavelength of $850 \mu \mathrm{m}$ over the original volume-limited survey. Fig. 1 shows the distribution of stars by age, emphasizing the survey bias towards younger targets.

The revised target list was assembled in 2011 from IRAS and Spitzer published data (Low et al. 2005; Beichman et al. 2006; Su et al. 2006; Rhee et al. 2007; Trilling et al. 2007, 2008; Bryden et al. 2009; Plavchan et al. 2009; Koerner et al. 2010; Morales et al. 2011; Zuckerman et al. 2011), unpublished data from Spitzer, Herschel DEBRIS and DUNES, the Herschel Guaranteed Time discs programme, Herschel GASPS (GAS in Protoplanetary Systems; 


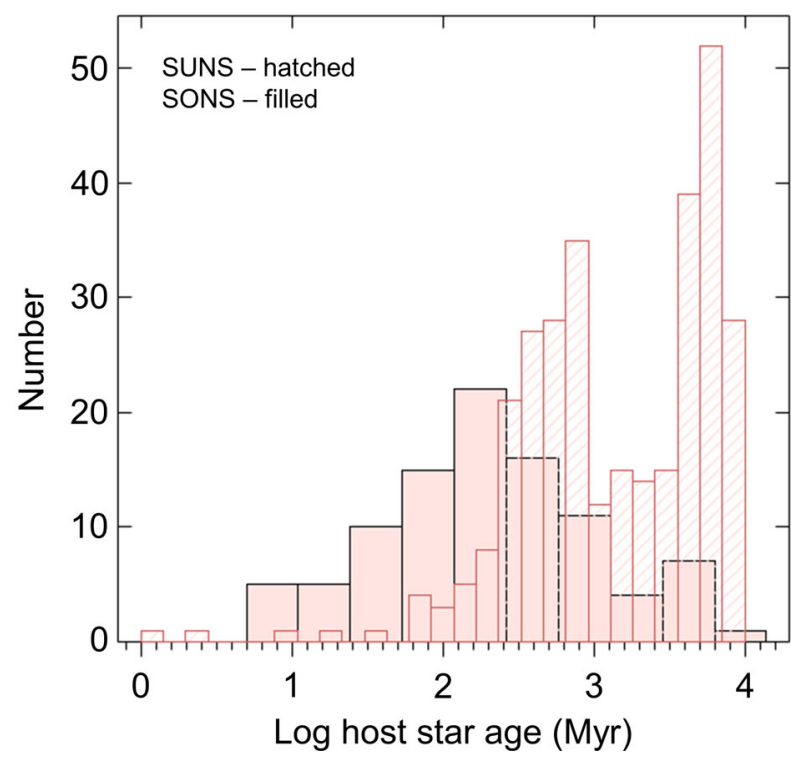

Figure 1. Histogram showing the difference in age distribution between the targets in the original SUNS (SCUBA-2 Unbiased Nearby Stars) survey of 500 stars and the re-scoped SONS survey of 115 targets.

Dent et al. 2013), and several smaller programmes on planet/disc hosts. Flux densities at $850 \mu \mathrm{m}$ were therefore predicted based on existing photometric data and a fit to the IR excess from the target. Simply assuming a standard blackbody spectrum would result in an overestimate of the $850 \mu \mathrm{m}$ flux by about a factor of 4 (Wyatt et al. 2007). Hence, particularly in the cases where few photometric points existed, predictions were based on modified blackbody spectra, $B_{v}\left(\lambda / \lambda_{0}\right)^{-\beta}$, assuming a critical wavelength, $\lambda_{0}=200 \mu \mathrm{m}$ and a dust emissivity index, $\beta=1.0$ (Wyatt 2008; Phillips 2011). Targets were then classified according to the likelihood of a $3 \sigma$ detection being achievable at $850 \mu \mathrm{m}$ with a flux density of at least $3 \mathrm{mJy}$.

Those sources classified as having guaranteed, likely or hard to quantify fluxes (i.e. with an unconstrained dust temperature), were retained if they were within $100 \mathrm{pc}$, had declinations between -40 and $+80^{\circ}$ and predicted $850 \mu \mathrm{m}$ fluxes of $>1 \mathrm{mJy}$ (or above $-60^{\circ}$ Dec. with predicted fluxes exceeding $15 \mathrm{mJy}$, to include several southern bright targets). It was accepted that there was still up a factor 3 uncertainty in the $850 \mu \mathrm{m}$ flux predictions for some targets. This uncertainty arises because the grain properties and size distribution are generally unknown; characterizing these was one of the key science goals of this survey. This method produced a candidate list of 115 targets (see Table 1) with 37 (i.e. one-third) of these in the guaranteed or likely detection categories. The selection criteria led to the expectation of a high detection rate, given the evidence of discs at multiple wavelengths for many targets. Fig. 2 shows the distribution of targets by host star spectral type and distance.

The survey was formally allocated $270 \mathrm{~h}$ of observing time on the JCMT, equally split between weather bands 2 and 3, equivalent to $225 \mathrm{GHz}$ zenith optical depths in the range $0.05-0.08$ for band 2 and $0.08-0.12$ for band $3 .^{1}$ The time allocated was sufficient to reach a $1 \sigma$ sensitivity limit of $1.4 \mathrm{mJy}$ at $850 \mu \mathrm{m}$ for each of the 115 fields. Furthermore, to maximize the chances of a disc detection a 'quick-look' approach to the observing methodology was adopted, in which each star was initially targeted for a minimum 1-h

\footnotetext{
${ }^{1}$ Zenith optical depths at $225 \mathrm{GHz}$ in the range $0.05-0.12$ correspond to line-of-sight precipitable water vapour levels of approximately $1-2.5 \mathrm{~mm}$.
}

observation block, typically achieving a $1 \sigma$ noise level of 1.5-2 mJy. Although SCUBA-2 operates simultaneously at wavelengths of 450 and $850 \mu \mathrm{m}$, the allocation of bands 2 and 3 weather meant that it was unlikely any significant number of discs would be detected at $450 \mu \mathrm{m}$. Such detections, however, were never a goal of the survey as it was planned to follow up possible $450 \mu \mathrm{m}$ detections with future observations, most likely requiring the best weather conditions ('band 1').

Any stars with detectable flux were immediately prioritized for more observing time (as needed, to boost the significance of the detection), with the possibility of returning to the others if and when time allowed. Although the time was scheduled as 3-4 night blocks on the telescope, roughly spaced every few months during the 3-yr survey period, the fact that the survey had targets all over the sky (as shown in Fig. 3) meant that SONS observations benefited from gaps in the schedules for the other legacy surveys. The broad sky distribution was the main reason that SONS became the first survey to be completed in terms of time in 2014 August. In addition to the 270-h formal allocation, the data presented in this paper also includes observations from the initial survey verification phase (2012 January) amounting to $26 \mathrm{~h}, 4.5 \mathrm{~h}$ from the SCUBA-2 Guaranteed Time allocation (PI: Holland) for observations of $\epsilon$ Eridani, a further $21 \mathrm{~h}$ from the survey extension programme in late 2014/early 2015, and $2 \mathrm{~h}$ each from the PI programmes M12AC17 and M13AC19 (PIs: Brenda Matthews and Christine Chen, respectively). The total observing time for the survey data was therefore $325.5 \mathrm{~h}$.

During the observing campaign, some observations were prioritized to confirm (or rule out) a previous marginal disc detection. Hence, more observing time was expended on a handful of disc candidates than was originally planned. Together with an overallocation of sources and fields around $05 \mathrm{~h}$ RA from the entire Legacy Survey programme, it became necessary to remove 15 targets from the list of 115 . These were mainly around $05 \mathrm{~h}$ RA (see Table 1), and included $\beta$ Pictoris as it has been well-characterized in the past at $850 \mu \mathrm{m}$ (e.g. Holland et al. 1998; Dent et al. 2014), and 14 further targets least likely to yield a disc detection based on the criteria outlined above.

\section{OBSERVATIONS AND DATA REDUCTION}

\subsection{Observations}

The SCUBA-2 camera (Holland et al. 2013) on the JCMT was used to take the survey data between 2012 January and 2015 February. The wavelengths of observation were 850 and $450 \mu \mathrm{m}$, where the primary beam sizes are 13.0 and 7.9 arcsec (measured Full-Width at Half Maximum; FWHM) (Dempsey et al. 2013). The data were taken exclusively using the constant speed DAISY observing mode, which maximizes the observing time in the central $3 \operatorname{arcmin}^{2}$ region of a field (Bintley et al. 2014). This mode is appropriate for compact sources of less than a few arcminutes in diameter and so is wellsuited to the observations of debris discs within the SONS survey. Each observation was taken as one continuous scan with a duration of approximately $30 \mathrm{~min}$. The data are saved as $30 \mathrm{~s} \mathrm{sub-scans,} \mathrm{with}$ each observation resulting in a total of 55 sub-scans, including a flatfield measurement at the start and end of each observation (Holland et al. 2013). The data were calibrated in flux density against the primary calibrators Uranus and Mars, and also secondary calibrators CRL 618 and CRL 2688 from the JCMT calibrator list (Dempsey et al. 2013), with estimated calibration uncertainties amounting to 20 and 7 per cent at 450 and $850 \mu \mathrm{m}$, respectively. Accurate telescope pointing was crucial to these observations and was regularly 
Table 1. The target list for the SONS survey. RA/Dec. positions and spectral types are from the SIMBAD data base (Wenger et al. 2000), and stellar distances from the Hipparcos catalogue (Perryman et al. 1997; van Leeuwen 2007). Stellar ages are referenced individually in Section 5.

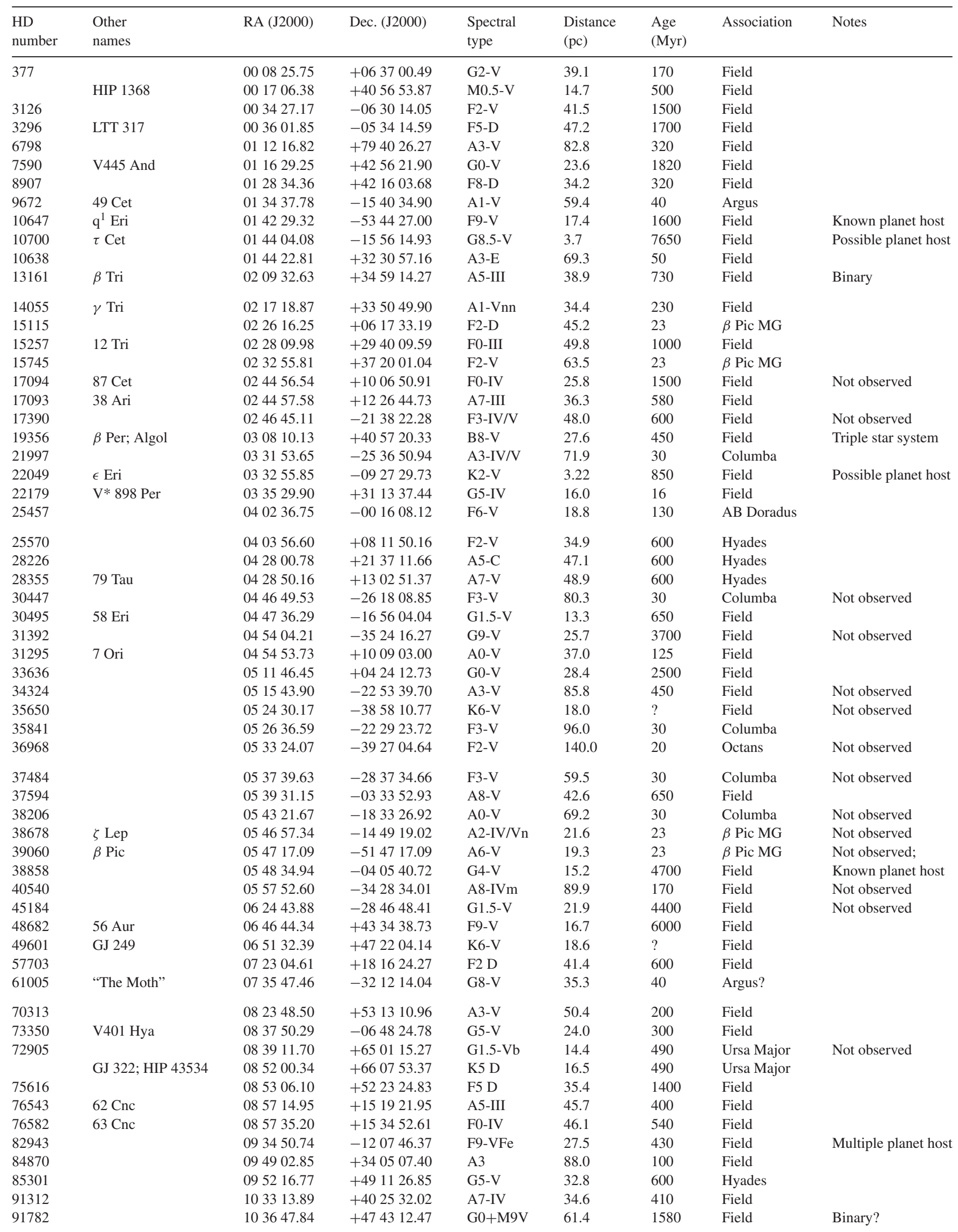


Table 1 - continued

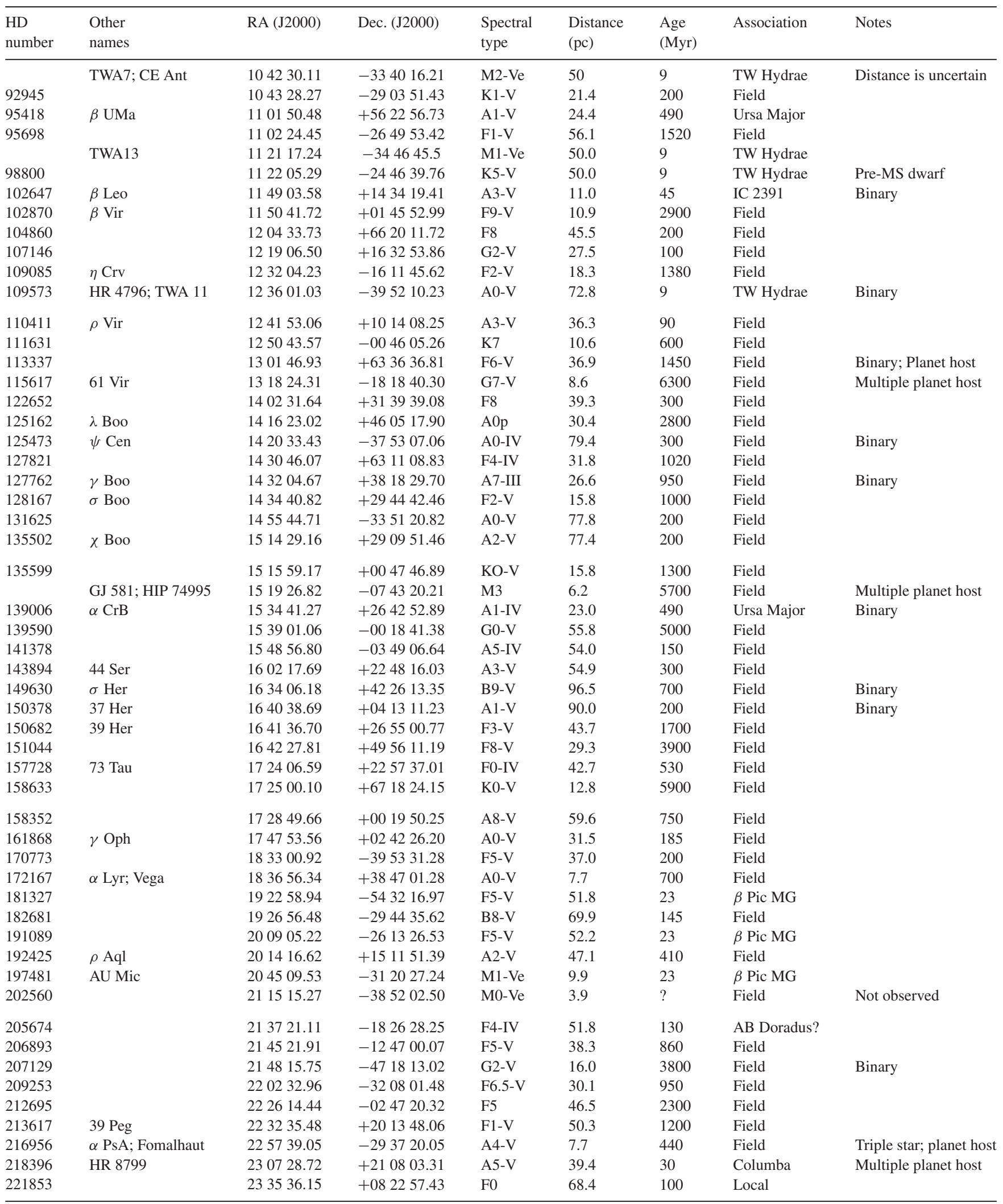



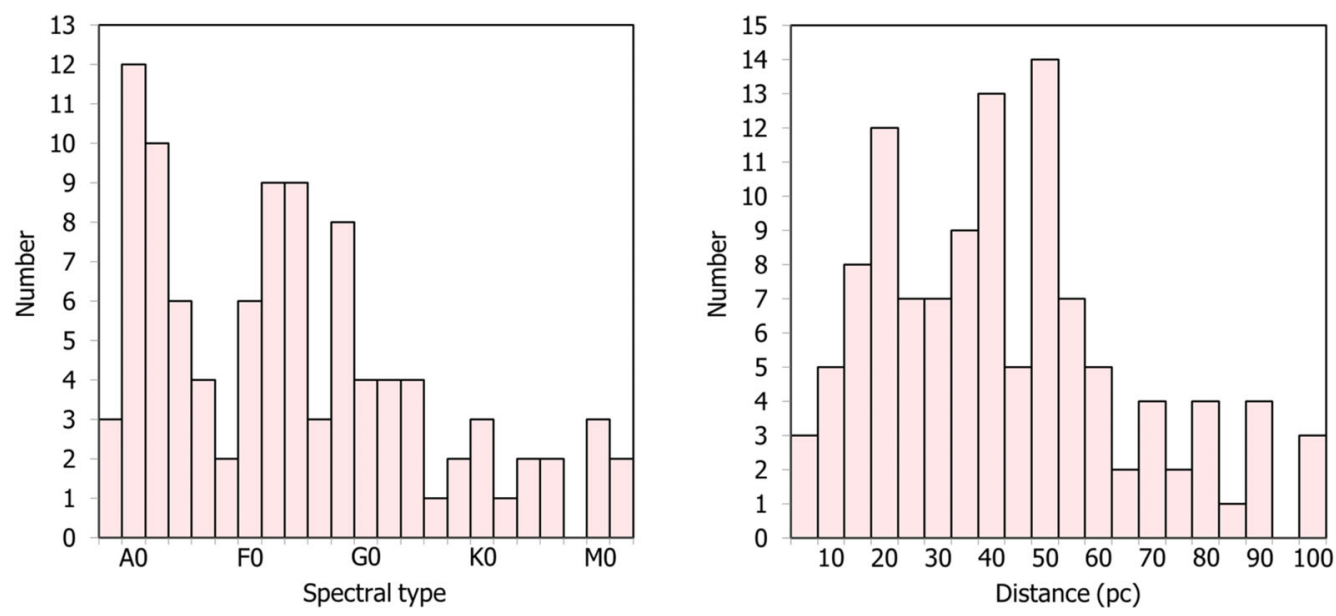

Figure 2. The distribution of SONS survey targets as a function of host star spectral type (left) and distance (right).

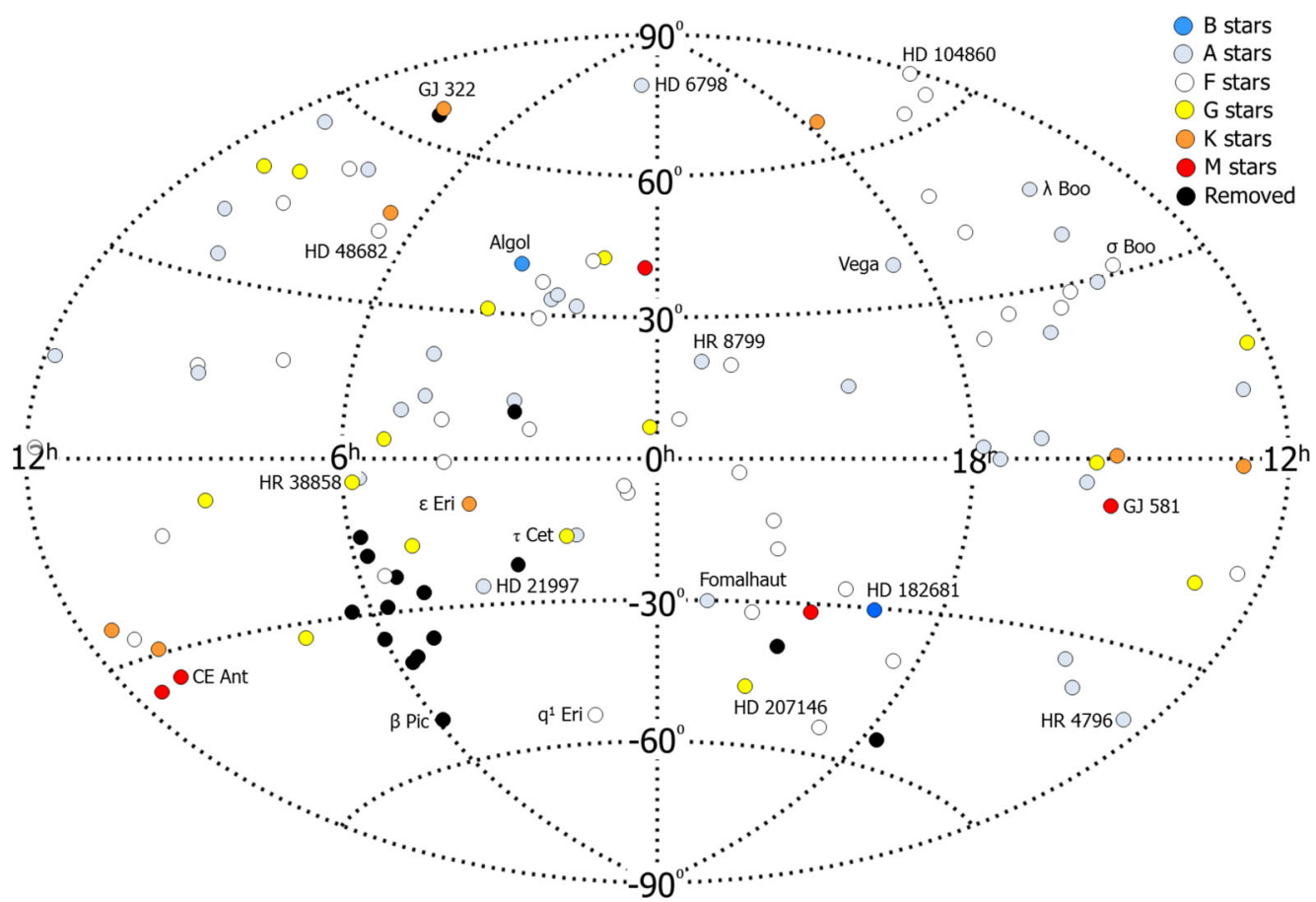

Figure 3. The distribution of SONS survey targets on the sky by spectral type. The spectral type for the stars is indicated by the colour table. Filled black circles are stars that were removed from the original 115 target list. For information, names for some of the stars are also labelled.

checked with reference to nearby bright sources (e.g. compact $\mathrm{H}$ II regions or blazars), with RMS pointing errors of less than 2 arcsec.

\subsection{Data reduction}

\subsubsection{Original approach: 'blank field'}

The data were reduced using the Dynamic Iterative Map-Maker within the Starlink SMURF package (Chapin et al. 2013) called from the ORAC-DR automated pipeline (Jenness \& Economou 2015). The original data reduction approach, used in the 'First Results' paper (Panić et al. 2013), saw the data heavily high-pass filtered at $1 \mathrm{~Hz}$, corresponding to a gradual spatial cut-off centred at $\sim 150$ arcsec for a typical DAISY scanning speed of $\sim 150 \operatorname{arcsec~} \mathrm{s}^{-1}$. The filtering was necessary to remove low-frequency noise originating from the detectors and readout electronics (Holland et al. 2013). To account for the attenuation of the signal, as a result of the time series filtering, the pipeline would re-make each map with a fake $10 \mathrm{Jy}$ Gaussian added to the raw data, but offset from the nominal map centre by 30 arcsec to avoid contamination with any detected source. The amplitude of the Gaussian in the output map gave the signal attenuation, and this correction was applied along with the flux conversion factor (FCF) derived from the calibrator observations. This method produced satisfactory results for unresolved compact sources but residual noise artefacts often remained in the images.

\subsubsection{Revised approach: 'zero masking'}

The heavy high-pass filtering led not only to residual instrumental noise but also to an under-estimation of the flux density as a function of source-scale size. This effect is not surprising as when sources become larger, they contain more power at lower 
frequencies, meaning that a fixed-frequency, high-pass filter will remove more flux. Hence, SONS adopted a revised map-maker configuration optimized for known position, compact and moderately extended sources. It used the technique of 'zero masking' in which the map is constrained to a mean value of 0 in all cases outside a radius of 60 arcsec from the centre of the field, for all but the final iteration of the map-maker (Chapin et al. 2013). The technique not only helped convergence in the iterative part of the map-making process but suppressed the large-scale ripples that can produce ringing artefacts. The results were more uniform, lower noise (by an average of $\sim 20$ per cent) final images, largely devoid of gradients and artefacts (Chapin et al. 2013). Each output map was regridded with 1 arcsec pixels at both wavelengths, and then smoothed with a 7 arcsec Gaussian using the Starlink package KAPPA recipe GAUSMOотH (Currie \& Berry 2013). FCFs were derived from the calibrator observations taken on the same night as the observations, reduced in exactly the same way as the source data, and applied to calibrate each map in flux density. FCFs were calculated based on the diameter of the observed disc. For unresolved discs the FCF was measured in a beam-sized aperture from the calibrator observation (often referred to as 'per beam' fluxes), whereas for resolved discs the conversion factor was based on an aperture diameter appropriate for the disc size (often referred to as 'integrated' or 'aperture' fluxes). The final images were made by coadding two or more maps using inverse-variance weighting implemented by the STARLINK package PICARD recipe MOSAIC JCMT IMAGES (Gibb, Jenness \& Economu 2013).

\subsubsection{Noise analysis}

Output data files from the map-maker were written in Starlink $N$ Dimensional Data Format (Jenness et al. 2015) and contain the rebinned image ('DATA' array) in terms of signal per output map pixel, together with a variance array representing the spread in values falling in a map pixel ('VARIANCE' array). Signal-to-noise (S/N) maps were produced (Starlink package KAPPA recipe MAKESNR) creating a new NDF file by dividing the DATA component by the square root of the VARIANCE array. This method, however, provides a noise estimate that is only representative of the true noise in an image if there are no residual features on a scale larger than approximately half a beam diameter. For unresolved discs the noise in an image was also obtained directly from the DATA array by taking integrated flux measurements within multiple beam-sized areas, spaced by 4 arcsec, from the central few arcminutes of the image. Similarly, for resolved discs the noise was estimated from apertures appropriate for the diameter of the disc, spaced by the one-third of the aperture diameter. In both cases the resulting flux distribution was fitted by a Gaussian (IDL HISTOGAUSS) with the noise level corresponding to the standard deviation of the fit. For the vast majority of the SONS survey measurements, both methods gave very similar results. This similarity was expected since the 'zero masking' technique is very effective at ensuring the final image is devoid of instrumental artefacts. The noise estimates reported in this paper are based on the measurements directly from the rebinned image (DATA array), and cases of residual instrumental artefacts in the images are discussed in Section 5 for individual targets. The errors reported for integrated fluxes (aperture photometry) are similarly derived from overlapping apertures of the same diameter used to determine the source flux.

\subsection{The Fomalhaut debris disc: a test case}

As discussed in Section 3.2, due to the excess low-frequency noise, one of the major challenges for the SONS survey was the reduction

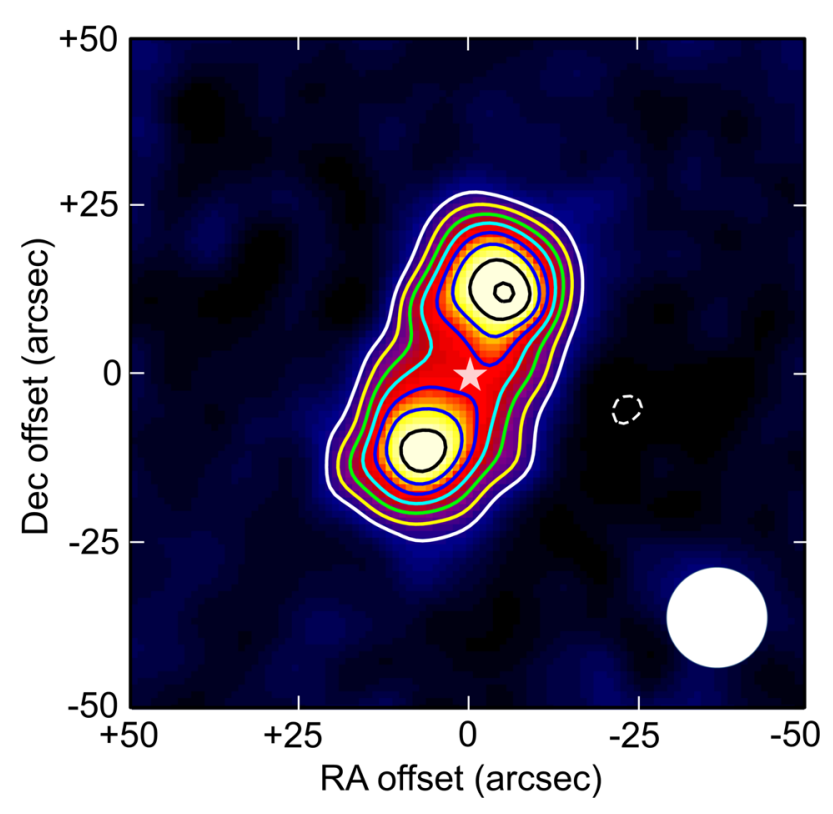

Figure 4. The Fomalhaut debris disc imaged by SCUBA-2 at $850 \mu \mathrm{m}$. This $\mathrm{S} / \mathrm{N}$ image is colour scaled from $-5 \sigma$ (black) to the maximum $\mathrm{S} / \mathrm{N}$ in the image at $26 \sigma$ (white). The dashed contour is at $-5 \sigma$ and the solid contours start at $5 \sigma$ and increase in $3 \sigma$ steps. The star symbol shows the position of Fomalhaut with respect to the disc (after proper motion corrections). The white circle represents the approximate FWHM beam size at $850 \mu \mathrm{m}$ after smoothing.

and calibration of moderately extended disc structures. The recovery of large-scale structure is a common issue for all observations undertaken with SCUBA-2. To test the robustness of the 'zero mask' data reduction method an extended, well-characterized debris disc was adopted as a test case. HD 216956 (Fomalhaut; $\alpha$ PsA) has been well-studied at all wavelengths from the optical to the millimetre, and the associated debris disc has a well-sampled SED. The disc is also one of the most extended so far discovered. The SCUBA$2 \mathrm{~S} / \mathrm{N}$ image of the Fomalhaut debris disc at $850 \mu \mathrm{m}$ (Fig. 4) shows an identical structure to previous submillimetre observations (Holland et al. 2003). Furthermore, the measured integrated flux within a 60 arcsec diameter aperture is $91 \pm 3 \mathrm{mJy}$, compared to the $97 \pm 5 \mathrm{mJy}$ measured from the SCUBA observations (Holland et al. 2003), consistent within the measured errors. Hence, the 'zero mask' data-reduction method works well for discs extending to at least 1 arcmin in diameter, although there remains some uncertainty for targets such as Vega (see Section 5.44).

\section{SURVEY OUTPUTS AND INTERPRETATION}

\subsection{Survey outputs}

The outputs of the survey are a catalogue of images and fluxes (including errors) for the 100 observed fields at $850 \mu$. $^{2}$ Table 2 lists the measured flux densities for all 100 stars and indicates whether the discs are resolved or unresolved by these observations. $\mathrm{S} / \mathrm{N}$ images for the detected fields are presented in Appendix A. Integration times ranged from a minimum of $1 \mathrm{~h}$ to a maximum of $10 \mathrm{~h}$. (The 5-h or longer observations were largely undertaken during the survey verification programme time, prior to the formal

2 The complete catalogue of images and SEDs for the entire sample of targets in the SONS survey, including the non-detections, is available online (doi:10.11570/17.0005). 
Table 2. Integration times and measured fluxes for the SONS survey sample. The column 'Disc' refers to whether the observed structure is unresolved ('P' - point-like) or resolved ('E' - extended compared to the beam diameter) at $850 \mu \mathrm{m}$. Fluxes are presented at both wavelengths with $3 \sigma$ and $5 \sigma$ upper limits quoted at 850 and $450 \mu \mathrm{m}$, respectively, in the case of a non-detected excess. Calibration uncertainties, as described in Section 3.1, are not included in the listed fluxes. For resolved (extended) structures the flux quoted is from aperture photometry (see Section 3.2.2) with the diameter of the aperture given in the individual source descriptions in Section 5.

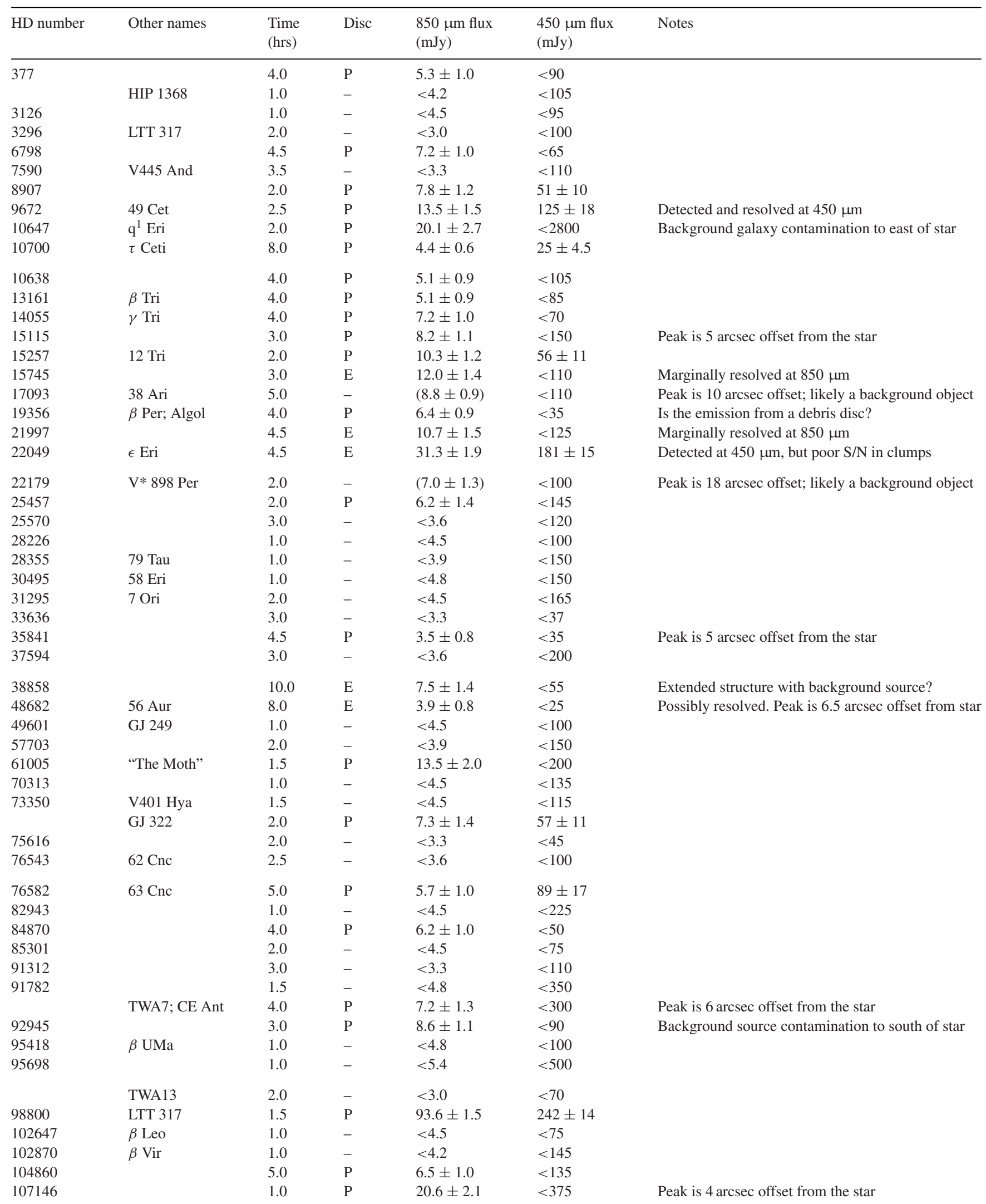


Table 2 - continued

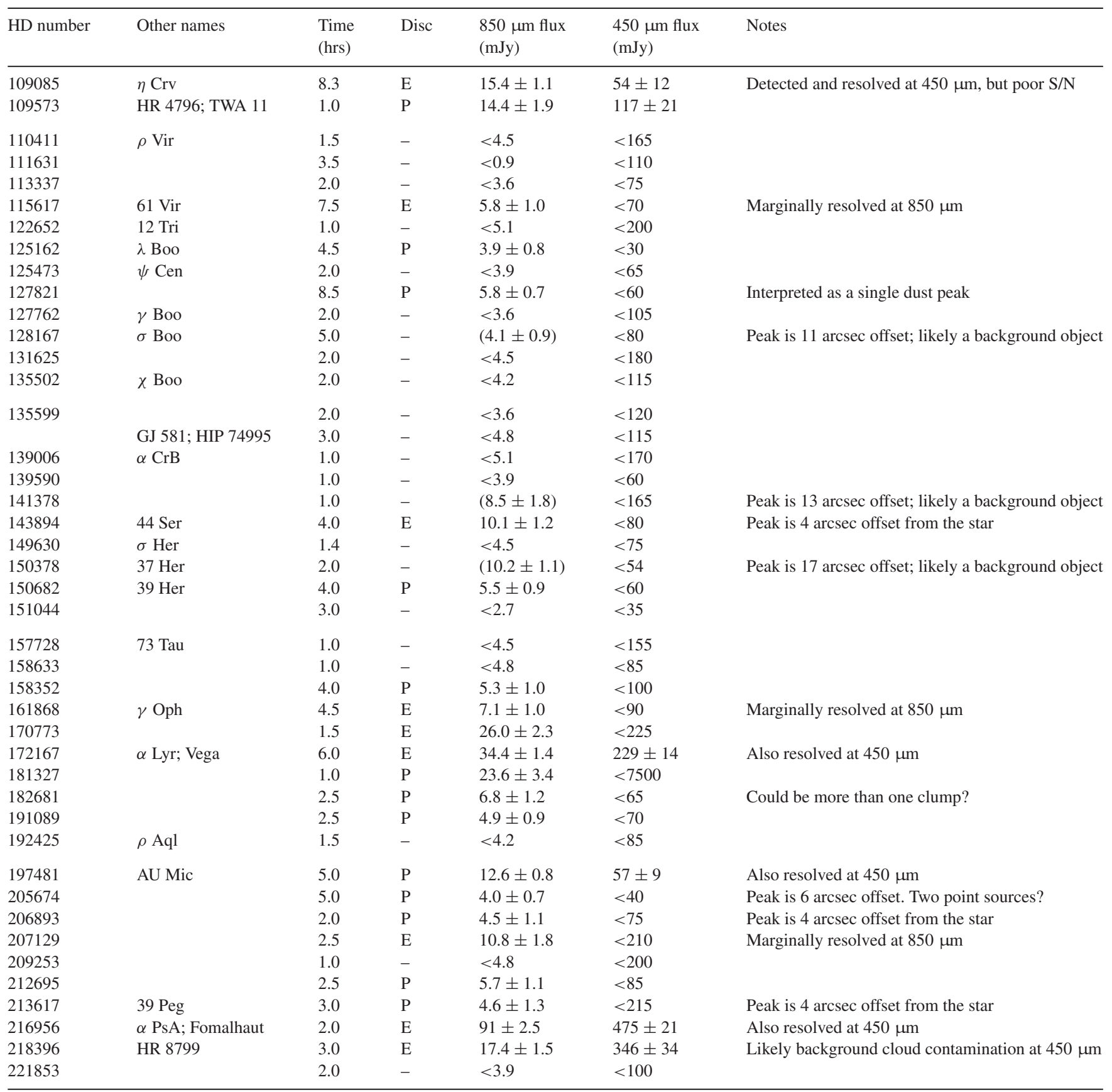

start of the SONS survey.) The results for each star are described in Section 5. As shown in Fig. 5, the RMS noise (determined from beam-sized apertures in all cases) within the inner 3 arcmin diameter region of each image decreases as $t_{\mathrm{obs}}^{-0.5}$, where $t_{\mathrm{obs}}$ is the integration time. The spread in RMS values is due to the relatively wide range of sky transmissions during the observations (i.e. the zenith sky opacity as defined by the allocated weather band combined with the airmass of the source at the time of the observation). Table 2 also lists five targets for which the peak is offset from the star by more than half a beam diameter $(\sim 7.5 \operatorname{arcsec}$ for the smoothed $850 \mu \mathrm{m}$ beam). In each of these cases the peak is interpreted as more likely to be a background object rather than a disc about the star (see Section 5).
Although the primary wavelength of observation for the survey was $850 \mu \mathrm{m}$, reflected by the allocation of weather bands 2 and 3 at the JCMT, a significant number of the targets showed flux excesses at $450 \mu \mathrm{m}$. Experience has shown that in bands 2 and 3 weather conditions, which is far from optimal for $450 \mu \mathrm{m}$ observations, the chances of a false detection are minimized by adopting a detection threshold of $5 \sigma$. Hence, whilst a level of $3 \sigma$ has been used when reporting $850 \mu \mathrm{m}$ results in this paper, a level of $5 \sigma$ has been adopted at $450 \mu \mathrm{m}$. A total of 14 targets in the sample reached this threshold, all of which were also detected at $850 \mu \mathrm{m}$. The $450 \mu \mathrm{m}$ photometry has been used to constrain further the fitting of the SED (Section 4.2) and offers improved angular resolution for extended structures (i.e. $\sim 10 \operatorname{arcsec}$ when the image is smoothed 


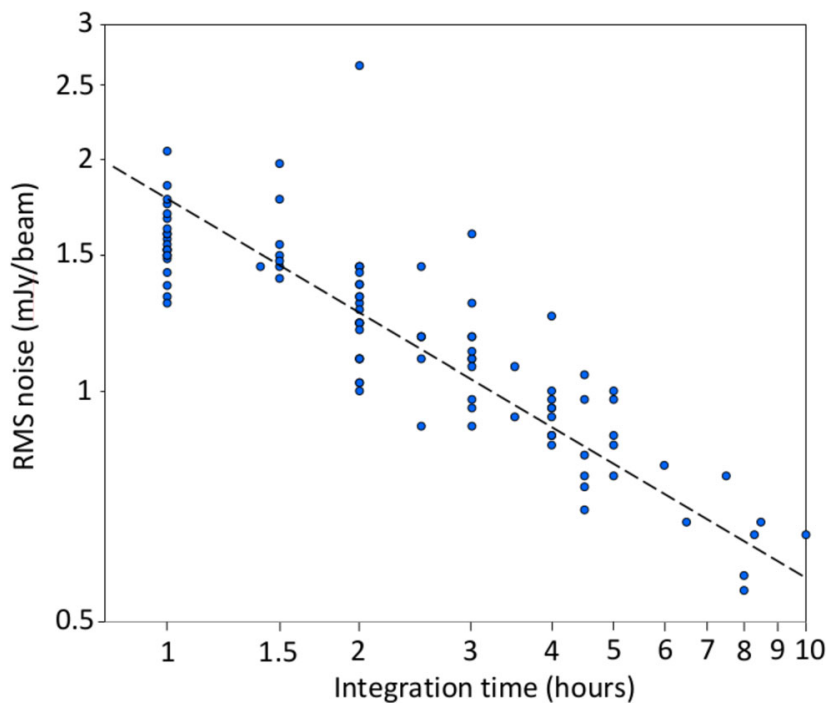

Figure 5. The measured RMS noise $\left(\mathrm{mJy}\right.$ beam $\left.^{-1}\right)$ at $850 \mu \mathrm{m}$ as a function of integration time $\left(t_{\mathrm{obs}}\right)$ for all 100 target fields in the SONS survey sample. The trend line indicates a noise decrease as $t_{\mathrm{obs}}^{-0.5}$.

with a 7 arcsec FWHM Gaussian). The results from the $450 \mu \mathrm{m}$ observations, where applicable, are noted in Table 2, and discussed in the individual source descriptions in Section 5.

\subsection{Dust temperature and emissivity}

Photometry for the target stars has been compiled from the optical to the millimetre from a wide variety of sources, including all-sky surveys such as IRAS (Moshir et al. 1990), Hipparcos (Perryman et al. 1997), 2MASS Point Source Catalog (Cultri et al. 2003), AKARI (Ishihara et al. 2010) and WISE (Wright et al. 2010), as outlined in Section 2. Further data are also provided by the surveys undertaken by Spitzer (including IRS data from the CASSIS data base) (e.g. Lebouteiller et al. 2011) and Herschel (e.g. Booth et al. 2013). Specific references to the photometric points provided for each target are given in the individual source descriptions in Section 5. The photometric data allow an SED to be assembled for each of the target stars, and these are shown in the figures presented in Appendix A, together with the $850 \mu \mathrm{m} \mathrm{S} / \mathrm{N}$ images.

The SED modelling adopted in this paper (Kennedy et al. 2012) has been successfully implemented for other surveys of debris discs, including the Herschel DEBRIS survey (e.g. Booth et al. 2013; Thureau et al. 2014). Photometry shortward of about $10 \mu \mathrm{m}$ was first used to model the stellar photospheric emission, and the estimated contributions to the remaining IR and submillimetre/millimetre photometric fluxes were then subtracted. These photometric points were then fitted by one- or two-component Planck functions in which a pure blackbody spectrum is modified beyond a critical wavelength, $\lambda_{0}$, as described in Section 2 . The best-fitting model was found by a least-squares minimization method. The model therefore accounts for inefficient emission by grains that are small relative to the wavelength of emission. The factor $\lambda_{0}$ is therefore representative of the grain size that dominates the emission spectrum, whilst the parameter $\beta$ is an index that describes the emissivity of the dust grains as well as being indicative of the size distribution of the dust.

In many cases, even with the $850 \mu \mathrm{m}$ photometry provided by the SONS survey, the sparse data coverage at submillimetre and millimetre wavelengths means that both $\lambda_{0}$ and $\beta$ are poorly constrained by the modelling. Moreover, in cases of only a single mea- surement beyond $160 \mu \mathrm{m}, \lambda_{0}$ and $\beta$ become strongly degenerate and no unique solution is possible. For some targets, a well-defined second component fit to the SED may possibly indicate the presence of multiple planetesimal belts (e.g. Morales et al. 2011; Chen et al. 2014; Kennedy \& Wyatt 2014). The main output parameters from the SED fitting (for one or more components), relevant to the interpretation of the IR/submm flux excess, are the dust temperature $\left(T_{\mathrm{d}}\right)$, critical wavelength $\left(\lambda_{0}\right)$ and the dust emissivity index $(\beta)$. These values are listed in Table 3 for the SONS survey sample, in which the derived $\beta$ 's are presented as a range of values (i.e. all values within the quoted range are possible).

\subsection{Disc radius and orientation}

The disc radius can also be estimated from derived parameters from the SED fit, assuming that the dust grains behave as a blackbody, and are uniformly distributed in a disc at a distance $R_{\mathrm{BB}}$ from the star (Wyatt 2008). In the cases where the emission is optically thin, the dust temperature can be used as a proxy for the radial separation from the star, which is given by:

$R_{\mathrm{BB}}=\left(\frac{278.3}{T_{\mathrm{d}}}\right)^{2} L_{*}^{0.5}$

where, if the dust temperature $\left(T_{\mathrm{d}}\right)$ is measured in kelvins, and the stellar luminosity $(L \star)$ in Solar luminosities, then $R_{\mathrm{BB}}$ is in astronomical units. The disc radii estimated from this method are presented in Table 3.

As already discussed in Section 4.2, the far-IR/submillimetre emission from discs is typically modelled using a modified blackbody spectrum. Therefore, the true radius of the disc is expected to be significantly larger in most cases (Rodriguez \& Zuckerman 2012; Booth et al. 2013; Pawellek et al. 2014), as is further discussed in Section 6.2. Hence, whilst modelling the SED provides a wealth of information about the disc properties, measuring the radius, directly from an image, allows better constraints to be placed on other physical properties such as the particle size distribution (Wyatt \& Dent 2002). For the cases in which discs are spatially resolved the $850 \mu \mathrm{m}$ (or $450 \mu \mathrm{m}$, as available) images are fitted using a 2D Gaussian function (IDL routine MPFIT2DFUn; Markwardt 2009) to estimate the radial extent of the disc. The fitted disc major and minor axes are deconvolved with the beam size (including the broadening effect of the smoothing factor), and the major axis multiplied by the distance of the star to give an estimate of the true disc radius according to:

$R_{\mathrm{fit}}=\frac{d}{2} \sqrt{\mathrm{FWHM}_{\mathrm{fit}}^{2}-\left(\mathrm{FWHM}_{\mathrm{beam}}^{2}+\mathrm{FWHM}_{\mathrm{smo}}^{2}\right)}$

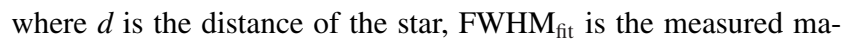
jor axis of the Gaussian fit, FWHM $_{\text {beam }}$ is the beam diameter (assumed circular and with a measurement variation of \pm 0.2 arcsec) and $\mathrm{FWHM}_{\text {smo }}$ is the Gaussian smoothing component (by default 7 arcsec). ${ }^{3}$

There are a few cases where the emission is not wellapproximated by a Gaussian profile. An example of this is Fomalhaut (HD 216956) where there are two equidistant lobes offset from the star position, and so the emission is not centrally concentrated. As discussed in Section 5.54, Herschel and Atacama Large Millimeter Array (ALMA) observations show that the emission is

\footnotetext{
${ }^{3}$ If the distance of the star is measured in parsecs and the FWHM beam in arcseconds, then the disc radius as specified in equation (2) will be in astronomical units.
} 
Table 3. The derived parameters from the SED fitting, measurements (and upper limits) from the radial profile fitting, and dust mass calculations for the SONS survey sample. Note that this list does not include the five 'extreme' offset cases, in which the flux peak is observed to be equal to, or greater than, 10 arcsec from the star (as indicated in Table 2). In the cases where the disc is unresolved the parameter $R_{\text {fit }}$ represents the upper limit to disc radius, corresponding to the beam radius at the distance of the star (see also the scale bars in the figures of Appendix A).

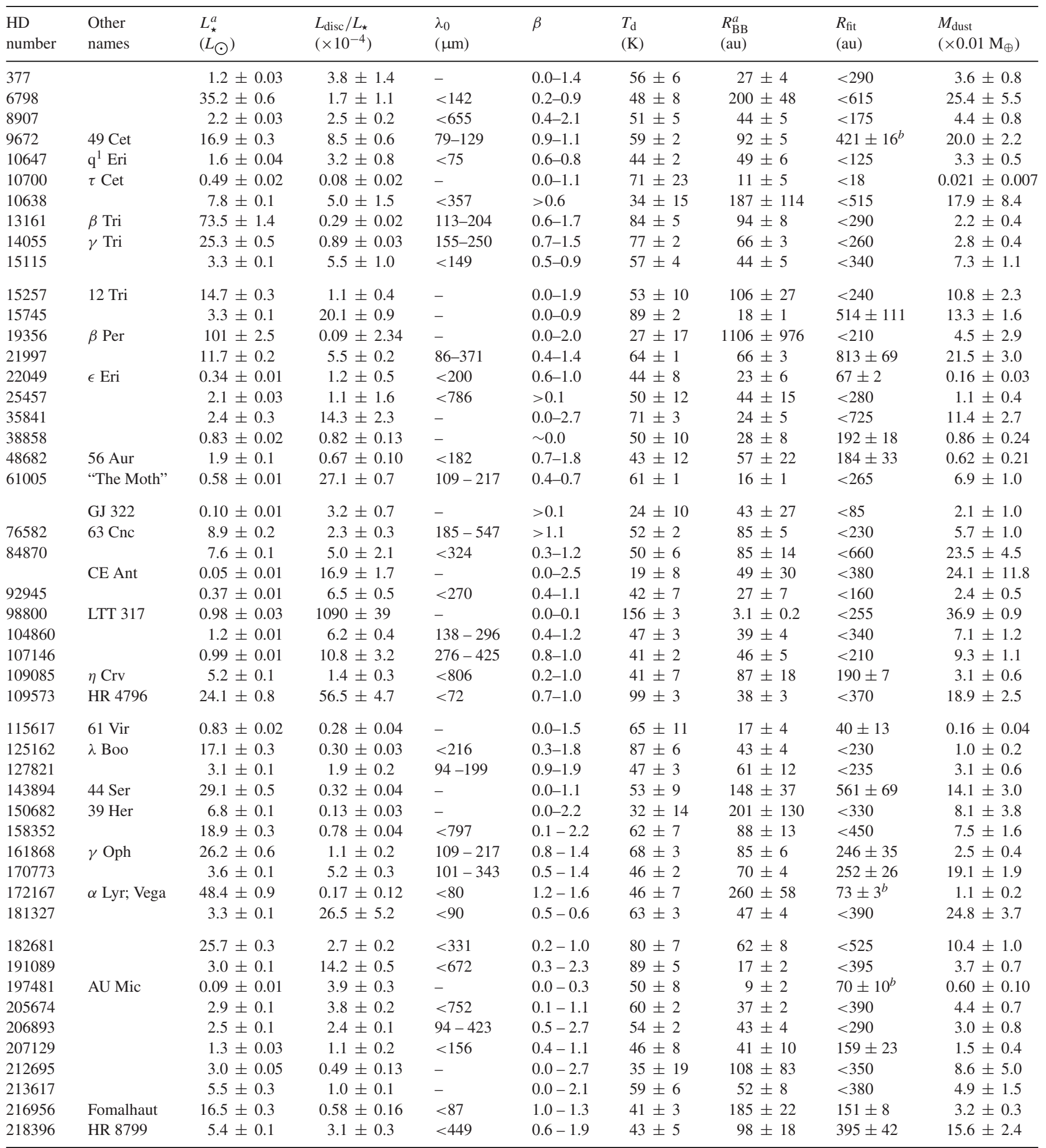

${ }^{a}$ The error quoted for $L^{\star}$ and $R_{B B}$ includes the uncertainty in the distance of the star from the Hipparcos catalogue (Perryman et al. 1997; van Leeuwen 2007). This is typically less than 5 per cent of the total error, which is dominated by errors in the fitting.

${ }^{b}$ Based on the fitted radius from the $450 \mu \mathrm{m}$ image. 

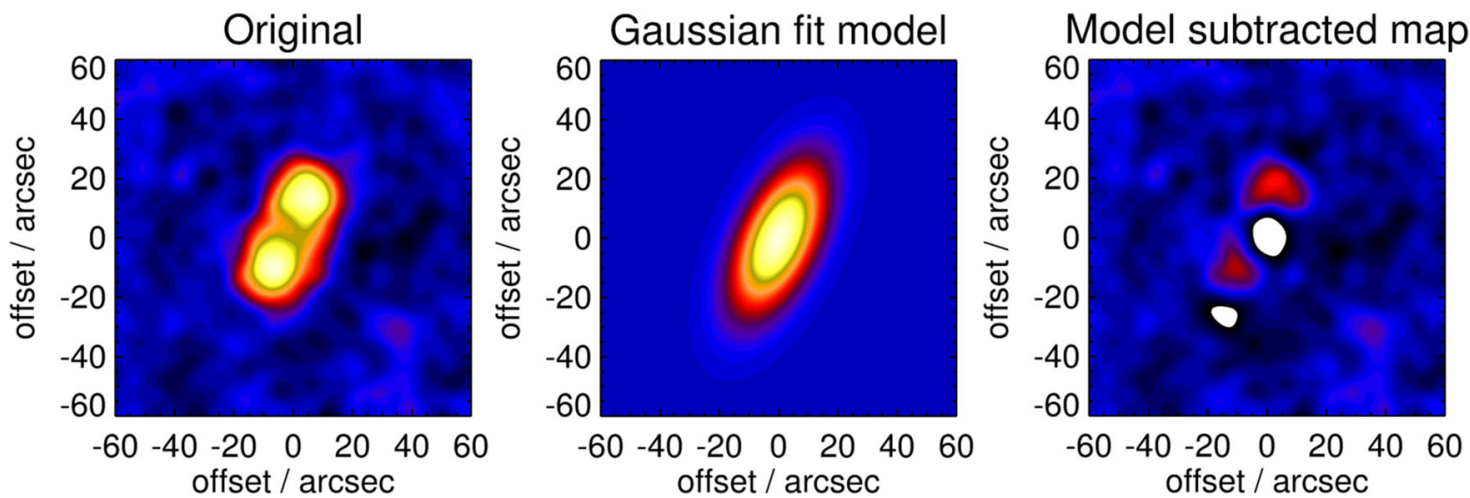

Figure 6. (left) The original $850 \mu \mathrm{m}$ observed image for HD 216956 (Fomalhaut); (centre) the Gaussian model is based on the fit to the radial extent; (right) the result of subtracting the observed image from the model map. Note that the residual emission at the star is not due to the star, but is due to oversubtraction, as the Gaussian model used is centrally peaked but the real flux distribution is not.

Table 4. Derived parameters from the radial extent fitting for resolved sources in the SONS survey sample. The waveband indicates the wavelength from which the measurements were made.

\begin{tabular}{|c|c|c|c|c|c|c|c|c|}
\hline $\begin{array}{l}\text { HD } \\
\text { number }\end{array}$ & $\begin{array}{l}\text { Other } \\
\text { names }\end{array}$ & $\begin{array}{l}\text { Waveband } \\
\qquad(\mu \mathrm{m})\end{array}$ & $\begin{array}{l}\text { Measured } \\
\text { major }(\operatorname{arcsec})\end{array}$ & $\begin{array}{l}\text { FWHM } \\
\text { minor }(\operatorname{arcsec})\end{array}$ & $\begin{array}{c}\text { Decconvolved } \\
\text { disc radius }(\operatorname{arcsec})\end{array}$ & $\begin{array}{l}\text { Disc radius } \\
R_{\text {fit }}(\mathrm{au})\end{array}$ & $\begin{array}{l}\text { Inclination } \\
\left({ }^{\circ}\right)\end{array}$ & $\begin{array}{c}\text { Position angle } \\
\left({ }^{\circ} \mathrm{E} \text { of } \mathrm{N}\right)\end{array}$ \\
\hline 9672 & \multirow[t]{2}{*}{49 Cet } & 450 & $17.5 \pm 0.5$ & $11.0 \pm 0.7$ & $7.1 \pm 0.5$ & $420 \pm 16$ & $74 \pm 13$ & $130 \pm 10$ \\
\hline 21997 & & 850 & $27.0 \pm 1.9$ & $<15$ & $11.3 \pm 1.9$ & $813 \pm 69$ & $\geq 48$ & $27 \pm 12$ \\
\hline 22049 & \multirow[t]{2}{*}{$\epsilon$ Eri } & 850 & $44.1 \pm 1.2$ & $40.2 \pm 1.3$ & $20.8 \pm 1.2$ & $67 \pm 4$ & $2 \overline{6} \pm 2$ & $61 \pm 3$ \\
\hline 38858 & & 850 & $29.3 \pm 2.4$ & $18.2 \pm 2.9$ & $12.7 \pm 2.4$ & $192 \pm 18$ & $65 \pm 18$ & $75 \pm 11$ \\
\hline 115617 & $61 \mathrm{Vir}$ & 850 & $17.5 \pm 3.0$ & $<15$ & $4.7 \pm 3.0$ & $40 \pm 13$ & $\geq 0$ & $66 \pm 25$ \\
\hline 143894 & 44 Ser & 850 & $25.2 \pm 2.5$ & $16.8 \pm 2.7$ & $10.2 \pm 2.5$ & $561 \pm 69$ & $67 \pm 24$ & $70 \pm 15$ \\
\hline 161868 & $\gamma \mathrm{Oph}$ & 850 & $21.5 \pm 2.2$ & $<15$ & $7.8 \pm 2.2$ & $246 \pm 35$ & $\geq 16$ & $75 \pm 17$ \\
\hline 170773 & & 850 & $20.1 \pm 1.4$ & $16.0 \pm 1.6$ & $6.8 \pm 1.4$ & $252 \pm 26$ & $63 \pm 17$ & $140 \pm 13$ \\
\hline \multirow[t]{2}{*}{172167} & \multirow[t]{2}{*}{$\alpha$ Lyr; Vega } & 450 & $21.6 \pm 0.7$ & $18.1 \pm 0.8$ & $9.5 \pm 0.7$ & $73 \pm 3$ & $34 \pm 2$ & $40 \pm 17$ \\
\hline & & 850 & $38.1 \pm 0.8$ & $32.3 \pm 0.9$ & $17.5 \pm 0.8$ & $135 \pm 3$ & 35 & $45 \pm 19$ \\
\hline
\end{tabular}

confined to a thin belt with the mid-point at a radius of $\sim 20$ arcsec from the star. Fig. 6 presents the results of the radial fit transposed into a model image and compared to the observed result. The modelsubtracted map shows a residual peak at the star position (and to a lesser extent in the south-east lobe) but the fit, for the purposes of this paper, is a reasonable representation of the overall disc size. The Fomalhaut case is somewhat of an extreme example, and for the vast majority of stars in the sample the fitting is well-suited to the disc morphology.

The inclination angle of the disc to the plane of the sky is derived from the axial ratio of the deconvolved major and minor axes fits (noting a $90^{\circ}$ angle degeneracy). Finally, the position angle (PA) of the major axis of the disc is measured north through east (also noting an angle degeneracy of $180^{\circ}$ ). The estimated disc radii, including upper limits for the cases in which the disc is unresolved, are given in Table 3. Full details of the measured major and minor axes radii, as well as the inclination and PAs, are presented in Table 4 for the 16 resolved discs in the SONS survey sample.

\subsection{Fractional luminosities}

The amount of dust in debris discs is often quantified in terms of the fractional luminosity, and can be determined from the SED fits to both the stellar photospheric and excess thermal emission. This quantity is defined as the ratio of the IR luminosity from the dust to that of the star, $f=L_{\mathrm{IR}} / L_{\star}$ (e.g. Wyatt 2008), and can be estimated from the wavelength and flux of the maximum in the emission spectra of the star and the disc, according to:

$f=\frac{F_{\mathrm{d}(\max )}}{F_{\star(\max )}} \frac{\lambda_{\star(\max )}}{\lambda_{\mathrm{d}(\max )}}$.

As equation (3) represents only an approximation to the fractional luminosity, $f$ is determined for the targets in this paper by the ratio of the integrated areas under the star and disc SED component fits. When there are two temperature components of the SED fit to the IR excess, then the fractional luminosity is derived from the sum of both. A defining property of a debris disc is that, in general, it has a fractional luminosity of $f<10^{-2}$ (Lagrange, Backman \& Artymowicz 2000) in contrast to protoplanetary discs, which have higher fractional luminosities. This criterion is certainly met by cool Edgeworth-Kuiper belt analogues, but falls down for stars at an earlier evolutionary phase where planet formation is believed to be ongoing, and where the flux excess tends to peak at mid-IR wavelengths (e.g. Melis et al. 2010; Fujiwara et al. 2012; Vican et al. 2016). The fractional luminosities for the SONS surveys discs are 
given in Table 3, and all but one (the exception being HD 98800) of the discs detected fall into this 'debris' classification.

\subsection{Dust masses}

Although the fractional luminosity can be converted into an estimate of dust mass by assuming all dust grains have the same diameter and density, dust masses are usually derived directly from the $850 \mu \mathrm{m}$ flux density measurement. Since the emission from debris discs is optically thin at these wavelengths, their mass is directly proportional to the emission, according to,

$M_{\mathrm{d}}=\frac{F_{\nu} d^{2}}{\kappa_{\nu} B_{v}\left(T_{\mathrm{d}}\right)}$

where $F_{v}$ is the measured flux density, $d$ is the distance of the target, $\kappa_{\nu}$ is the dust opacity that is assumed to be $1.7 \mathrm{~cm}^{2} \mathrm{~g}^{-1}$ at $850 \mu \mathrm{m}$ in accordance with similar studies (Pollack et al. 1994; Dent et al. 2000) and $T_{\mathrm{d}}$ is the dust temperature derived from the SED fit. In the Rayleigh-Jeans limit, the mass becomes a linear function of temperature and so equation (4) reduces to,

$M_{\mathrm{d}}\left[M_{\oplus}\right]=5.8 \times 10^{-10} \frac{F_{v}[\mathrm{mJy}] \mathrm{d}[\mathrm{pc}]^{2} \lambda[\mu \mathrm{m}]^{2}}{\kappa_{v}\left[\mathrm{~cm}^{2} \mathrm{~g}^{-1}\right] T_{\mathrm{d}}[\mathrm{K}]}$

The calculated dust masses are summarized in Table 3 for the SONS survey sample, with quoted uncertainties based on the errors in the $850 \mu \mathrm{m}$ flux and fitted dust temperature only.

\section{INDIVIDUAL TARGETS DISCUSSION}

This section of the paper provides a discussion of each of the targets for which emission was detected in the vicinity of the star. Each subsection summarizes the new SONS survey results at $850 \mu \mathrm{m}$ (and $450 \mu \mathrm{m}$, if available) within the context of previous observations, as well as the results of the modelling of the SED and the estimation of the dust mass from the $850 \mu \mathrm{m}$ flux. Full details, including estimated errors on the modelled and calculated parameters such as $T_{\mathrm{d}}, R_{\mathrm{BB}}$, $R_{\mathrm{fit}}, \beta$ and $M_{\mathrm{d}}$, are given in Tables 3 and 4 .

\subsection{Hd 377}

HD 377 is a Solar-type star (G2V) at a distance of $39.1 \mathrm{pc}$ with an estimated age of around $170 \mathrm{Myr}$ (Chen et al. 2014), but could be as young as $32 \mathrm{Myr}$ (Hillenbrand et al. 2008) or as old as $250 \mathrm{Myr}$ (Choquet et al. 2016). The SONS survey image, as shown in Fig. A1a, reveals emission centred on the stellar position with flux density of $5.3 \pm 1.0 \mathrm{mJy}$ at $850 \mu \mathrm{m}$. (This value is revised slightly higher from the result reported in Panić et al. 2013.) Interpreting this peak as an unresolved disc about the star gives an upper limit to the radius of $290 \mathrm{au}$.

HST/NICMOS imaging of HD 377 shows an edge-on disc at a PA of $47^{\circ}$ with a radius of $2.2 \operatorname{arcsec}(\sim 86 \mathrm{au})$ (Choquet et al. 2016). The disc has also been resolved using the Smithsonian Millimeter Array (SMA) (Steele et al. 2016) with a $850 \mu \mathrm{m}$ flux of $3.5 \pm 1 \mathrm{mJy}$, just consistent with the SONS result, and a deconvolved disc radius of $47 \mathrm{au}$ at a PA of $30^{\circ}$. Although the mid-far-IR region is reasonably well characterized by Spitzer (Chen et al. 2014), there are few constraining points in the submillimetre/millimetre. Previous SED modelling suggested a two-component fit deriving 'warm' and 'cold' elements with dust temperatures of 240 and $57 \mathrm{~K}$ (Chen et al. 2014). Using photometry at $1.2 \mathrm{~mm}$ from the IRAM $30 \mathrm{~m}$ telescope, a dust mass of $0.058 \mathrm{M}_{\oplus}$ was derived (Roccatagliata et al. 2009).
Modelling of the SED with the new $850 \mu \mathrm{m}$ flux density measurement included gives a cold component dust temperature of $56 \mathrm{~K}$, but the poorly sampled long-wavelength slope means that $\beta$ is only constrained to be less than a value of 1.4. The estimated dust mass for the cold component of $0.036 \mathrm{M}_{\oplus}$ is lower than the IRAM result, but is just consistent within the measurement errors.

\subsection{Hd 6798}

HD 6798 is a luminous A3 star in Cepheus lying at a distance of $83 \mathrm{pc}$ and an estimated age of around $320 \mathrm{Myr}$ but with an uncertainty spanning the range from 260 to $400 \mathrm{Myr}$ (Moór et al. 2006). An emission peak is seen at $850 \mu \mathrm{m}$ with a flux density of $7.2 \pm 1.0 \mathrm{mJy}$ (Fig. A1b). As an unresolved disc, the upper limit to the radius is $615 \mathrm{au}$.

The SED contains flux measurements from IRAS and Herschel/PACS (archival data) in the range 25-160 $\mu \mathrm{m}$ but no other long wavelength points. Modelling suggests, as in the case of HD 377, that there are both warm and cold disc components with the former having a dust temperature of $203 \mathrm{~K}$. The fit to the far-IR/submillimetre detected cold disc component gives a dust temperature of $48 \mathrm{~K}$ and constrains $\beta$ to be between 0.2 and 0.9 . The dust mass is estimated to be $0.25 \mathrm{M}_{\oplus}$.

\subsection{Hd 8907}

HD 8907 is an F8 star in Andromeda at a distance of 35 pc and with an estimated age of around $320 \mathrm{Myr}$ (Hillenbrand et al. 2008), although there is significant uncertainty with lower and upper limits put at 110 and $870 \mathrm{Myr}$, respectively (Moór et al. 2006). The peak in emission is coincident with the star position, and is detected at a flux density of $7.8 \pm 1.2 \mathrm{mJy}$ at $850 \mu \mathrm{m}$ (Fig. A1c), somewhat higher than the previous SCUBA measurement of $4.8 \pm 1.2 \mathrm{mJy}$ (Najita $\&$ Williams 2005). Emission was also detected (and unresolved) at $450 \mu \mathrm{m}$ with a flux density of $51 \pm 10 \mathrm{mJy}$. The $450 \mu \mathrm{m}$ image with $850 \mu \mathrm{m}$ contours overlaid is shown in Fig. 7, showing both peaks to be coincident. Based on the $450 \mu \mathrm{m}$ image, and interpreting the peak as a disc about the star, gives an upper limit to the radius of $175 \mathrm{au}$.

The SED is well characterized in the IR and submillimetre/millimetre, and observations at $1.2 \mathrm{~mm}$ using the SMA (Steele et al. 2016) resolve the disc with a measured radius of 54 au at a $\mathrm{PA}$ of $55^{\circ}$. The SONS measurements help to constrain the SED fit, with a dust temperature of $51 \mathrm{~K}$, and a $\beta$ value in the range $0.4-2.1$. The dust mass is estimated to be $0.044 \mathrm{M}_{\oplus}$.

\subsection{Ceti (HD 9672)}

HD 9672 (49 Ceti) is a young A1 star in Cetus and a member of the Argus Association, at a distance of $59 \mathrm{pc}$ with an estimated age of $40 \mathrm{Myr}$ (Torres et al. 2008) but could be as young as $20 \mathrm{Myr}$ (Chen et al. 2014). At $850 \mu \mathrm{m}$ the detected emission at the stellar position appears to be unresolved (Fig. A1d) with a flux density of $13.5 \pm 1.5 \mathrm{mJy}$. At $450 \mu \mathrm{m}$, however, the emission morphology is elongated at a PA of $130^{\circ}$ with a flux density of $125 \pm 18 \mathrm{mJy}$, as shown in Fig. 8 (Greaves et al. 2016). The measured disc FWHM of 17.5 arcsec (a deconvolved radius of 7.1 arcsec, corresponding to $\sim 420 \mathrm{au}$ ) at $450 \mu \mathrm{m}$, indicates that the structure is likely a disc about the star, that could also be marginally resolved at $850 \mu \mathrm{m}$.

Herschel/PACS observations at $70 \mu \mathrm{m}$ reveal a resolved disc of radius 250 au with a PA of $105^{\circ}$ (Roberge et al. 2013). Recent 


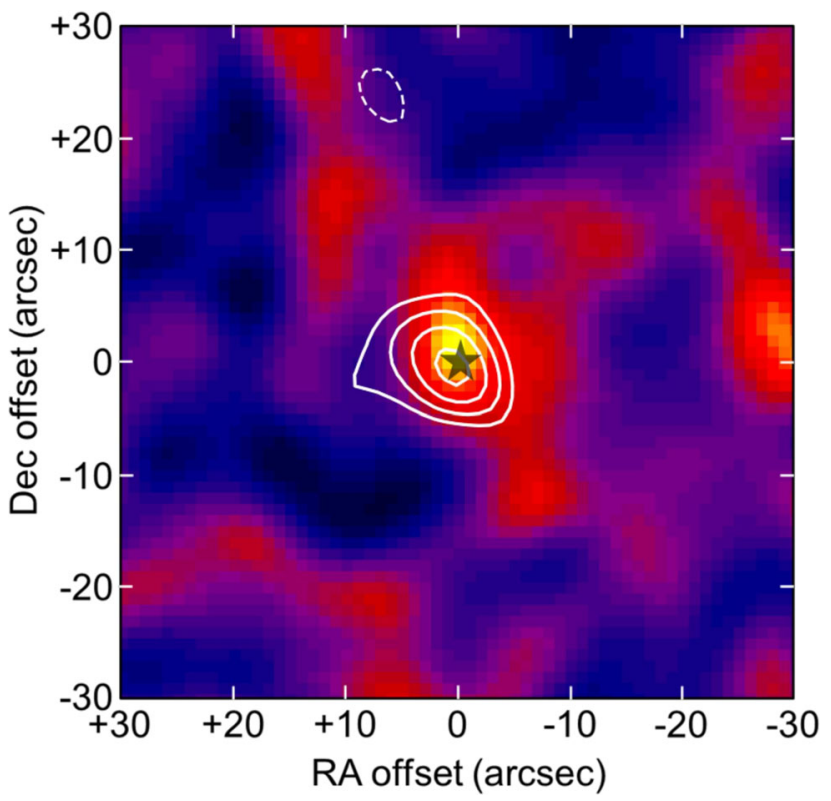

Figure 7. The $450 \mu \mathrm{m} \mathrm{S/N}$ image from observations of HD 8907 with contours from the $850 \mu \mathrm{m}$ image overlaid. The colours are scaled from $-3 \sigma$ to the maximum $\mathrm{S} / \mathrm{N}$ in the image $(\sim 6 \sigma)$. The contours start at $-3 \sigma$ (dashed white) and then solid colours from $3 \sigma$ to the maximum in $1 \sigma$ steps. The star symbol represents the position of the star with respect to the flux peak.

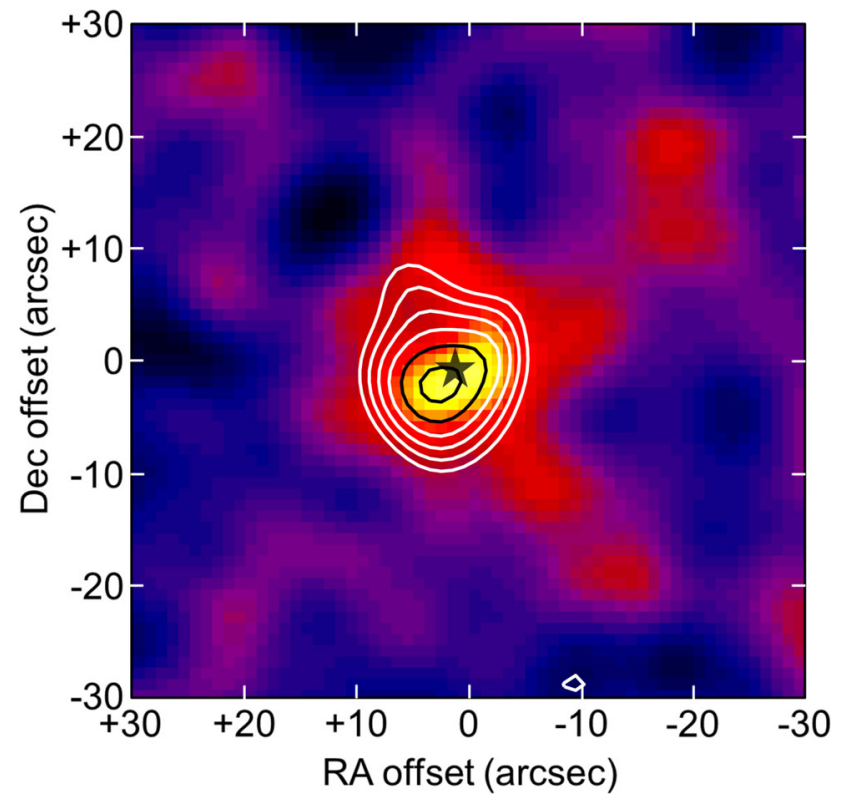

Figure 8. The $450 \mu \mathrm{m}$ image from observations of 49 Ceti (HD 9672) with contours from the $850 \mu \mathrm{m}$ image overlaid. The contours and symbols are as described in Fig. 7.

ALMA observations also resolve the disc with a PA of $107^{\circ}$ identifying dust that extends from just a few to around 300 au from the star (Hughes et al. 2017). The fact that this is significantly less in extent than implied by the SONS result at $450 \mu \mathrm{m}$ remains an open issue. The observed structure has been modelled as having two components: an inner disc extending to a radius of $60 \mathrm{au}$ (and depleted at less than $30 \mathrm{au}$ ) (Wahhaj, Koerner \& Sargent 2007) and an outer disc of radius up to 400 au (Greaves et al. 2016). Scat- tered light images, from $H S T /$ NICMOS and coronograhic $H$-band images using VLT/SPHERE, show the outer disc extending from 1.1 to 4.6 arcsec $(\sim 65-250 \mathrm{au})$ with an inclination angle of $73^{\circ}$ and a PA of $106-110^{\circ}$ (Choquet et al. 2017). In addition, the system has a well-known molecular and atomic gas reservoir, which was originally purported to be consistent with the properties of a lowmass protoplanetary disc (Zuckerman, Forveille \& Kastner 1995). The age estimate for the system (likely to be $40 \mathrm{Myr}$ ), however, suggested that it was more likely the gas had a secondary origin, perhaps involving a high rate of comet destruction given the large observed dust mass (Zuckerman \& Song 2012; Hughes et al. 2017).

The disc is well-characterized in the far-IR, with observations from Spitzer and Herschel contributing to points in the SED. In the millimetre the measured flux from IRAM at $1.3 \mathrm{~mm}$ of $13.9 \pm 2.5 \mathrm{mJy}$ (Walker \& Butner 1995) is significantly higher than expected based on the SED fit to the far-IR and submillimetre points. The reason for this is unknown. The SED, including $9 \mathrm{~mm}$ photometry from the VLA (MacGregor et al. 2016a) but not using the IRAM $1.3 \mathrm{~mm}$ photometry, is modelled with a two-component fit. The 'warm' (inner) element has a characteristic temperature of $165 \mathrm{~K}$. The dominant 'cold' (outer) component has a dust temperature of $59 \mathrm{~K}$, a $\beta$ value of between 0.9 and 1.1 , and a calculated dust mass of $0.20 \mathrm{M}_{\oplus}$.

\section{$5.5 q^{1}$ Eridani (HD 10647)}

$\mathrm{q}^{1}$ Eridani is a nearby F9 star at a distance of $17 \mathrm{pc}$ and with an estimated age of $1600 \mathrm{Myr}$ (Chen et al. 2014). There is, however, considerable uncertainty in the age with estimates ranging from 300 to $7000 \mathrm{Myr}$ (Moór et al. 2006). Even though the southerly declination of HD 10647 was quite a challenge for observing with the JCMT, a structure, peaking in emission at the star and extending eastwards, was detected at $850 \mu \mathrm{m}$ (Fig. A2a). The measured integrated flux of $30.3 \pm 3.9 \mathrm{mJy}$, measured in a 50 arcsec diameter aperture centred on the star, is just consistent with the APEX/LABOCA result of $39.4 \pm 4.1 \mathrm{mJy}$ at $850 \mu \mathrm{m}$ (Liseau et al. 2008), the data for which also show a roughly eastward extension to the disc. The structure is resolved within the 15 arcsec beam, and interpreted as a disc with a deconvolved radius of $9.0 \operatorname{arcsec}(\sim 155 \mathrm{au})$ at a PA of $72^{\circ}$, dominated by the eastward extension.

The IR excess was first detected by IRAS (Stencel \& Backman 1991). The system has one known Jupiter-mass planet (HD 10647 b; Butler et al. 2006) orbiting at a semimajor axis of 2 au, so is unlikely to have any significant influence on a dust disc of radius $>100$ au. HST/ACS coronography revealed an 7.0-8.2 $\operatorname{arcsec}(\sim 120-140 \mathrm{au})$ radius disc in scattered light with a PA of $56^{\circ}$ (Stapelfeldt et al. 2007). Herschel/PACS also resolved the disc with a PA of $54^{\circ}$ and a beam-deconvolved radius of $6.7 \times 3.8 \operatorname{arcsec}$ at $160 \mu \mathrm{m}(\sim 115 \times$ $65 \mathrm{au}$; Liseau et al. 2010). In the $850 \mu \mathrm{m}$ SONS image, the disc appears moderately extended compared to the beam in a roughly easterly direction with most of the flux concentrated at the position of the star. Within the $4 \sigma$ contour the PA of the disc in the $850 \mu \mathrm{m}$ SONS image is $\sim 65^{\circ}$, agreeing reasonably well with both the $H S T$ and Herschel observations, and on a similar scale $(\sim 15 \operatorname{arcsec})$ at least in the north-east direction. (There is also a hint of an extension to the south-west.)

A more plausible explanation is that the eastward extension seen in the SCUBA-2 and LABOCA images is caused by a background object. This extra flux would explain why both $850 \mu \mathrm{m}$ values are slightly high based on the SED model fit to the far-IR photometric points, and why there is not a symmetric disc seen about the star, as in the HST and Herschel images. In the Herschel/PACS $70 \mu \mathrm{m}$ 
image, there is evidence of an isolated source 20 arcsec to the east of the main flux peak (Liseau et al. 2010), which aligns well with the eastward extension seen at $850 \mu \mathrm{m}$. A similar extension is also seen in the Herschel/PACS $160 \mu \mathrm{m}$. Hence, in this paper, the IR excess flux surrounding $\mathrm{q}^{1}$ Eri has been re-interpreted as an unresolved disc with a flux density of $20.1 \pm 2.7 \mathrm{mJy}$ at $850 \mu \mathrm{m}$. (This is the flux measured in a beam-sized aperture, centred on the star position.) The SED is well-constrained by Spitzer, Herschel, the long submillimetre points (including SCUBA-2 at $850 \mu \mathrm{m}$ ) and $6.8 \mathrm{~mm}$ photometry from ACTA (Ricci et al. 2015b), to give a dominant temperature component at $44 \mathrm{~K}$, a well-constrained $\beta$ between 0.6 and 0.8 , and an estimated dust mass of $0.033 \mathrm{M}_{\oplus}$. An upper limit to the disc radius from the $850 \mu \mathrm{m}$ image, assuming an unresolved source, is $125 \mathrm{au}$.

\section{$5.6 \tau$ Ceti (HD 10700)}

HD 10700 ( $\tau$ Ceti) is a nearby G-type star, believed to be very similar to our Sun, at a distance of $3.7 \mathrm{pc}$ with an estimated age of $7650 \mathrm{Myr}$, but with an uncertainty in the range 6130-8500 Myr (Pagano et al. 2015). The SONS $850 \mu \mathrm{m}$ image shows a peak in emission at the stellar position with a flux density of $4.4 \pm 0.6 \mathrm{mJy}$ (Fig. A2b) of which an estimated $1 \mathrm{mJy}$ is emission from the photosphere. Interpreting the emission morphology as a disc gives an upper limit to the radius from the $850 \mu \mathrm{m}$ image of $27 \mathrm{au}$.

The IR excess has been known since the early days of IRAS and was later confirmed by ISO (Habing et al. 2001). $\tau$ Ceti was first imaged by SCUBA (Greaves et al. 2004), which suggested a disc extending some 55 au from the star, and identified the structure as possibly being a massive Edgeworth-Kuiper Belt analogue. The disc was subsequently resolved by Herschel at $70 \mu \mathrm{m}$ and $160 \mu \mathrm{m}$ (possibly also at $250 \mu \mathrm{m}$ ) with the peak emission occurring at a radius of $5 \operatorname{arcsec}(\sim 20 \mathrm{au})$ in the photosphere-subtracted image at $70 \mu \mathrm{m}$ (Fig. 3 of Lawler et al. 2014), and modelled as a wide annulus with an inner edge between 1 and 10 au and an outer edge at $\sim 55$ au inclined from a face-on orientation by $35 \pm 10^{\circ}$ (Lawler et al. 2014). More recently $1.3 \mathrm{~mm}$ interferometric observations with ALMA have revealed a nearly face-on belt of cold dust at a radius of 44 au with a PA of $90^{\circ}$ surrounding an unresolved central source at the stellar position (MacGregor et al. 2016a). Modelling of the belt suggests an inner edge of 6 au, consistent with Herschel observations. There are also five candidate planets orbiting $\tau$ Ceti, all of which are believed to lie within a radius of 1.35 au of the star (Tuomi et al. 2013). Whilst the outermost planet may have some influence on the disc inner edge, the majority of the disc extending from a few au to over 50 au will be largely unaffected by the planetary system. The existence of the planetary system, however, remains unconfirmed, following recent work in which periodic signals were not detected from Doppler measurements obtained in the California Planet Survey (Howard \& Fulton 2016).

The disc is also detected at $450 \mu \mathrm{m}$ within the SONS survey with a flux density of $25.2 \pm 4.5 \mathrm{mJy}$ (Fig. 9) including an estimated $5 \mathrm{mJy}$ from the photosphere. The separation of the 450 and $850 \mu \mathrm{m}$ peaks is $\sim 5$ arcsec, consistent within the expected statistical uncertainties (see Section 6.4), with the star positioned equidistant between the two peaks. The upper limit to the radius from the $450 \mu \mathrm{m}$ image of $18 \mathrm{au}$. Lawler et al. (2014) suggested that since no SED model can adequately fit both the Herschel/PACS and SCUBA/SCUBA-2 points, a separate, cooler disc component may be required to explain the submillimetre observations. The SED shown in Fig. A2 b is the best single fit to all the points beyond $70 \mu \mathrm{m}$, and results in a singletemperature component of $71 \mathrm{~K}$ (but with large errors of $\pm 23 \mathrm{~K}$ ),

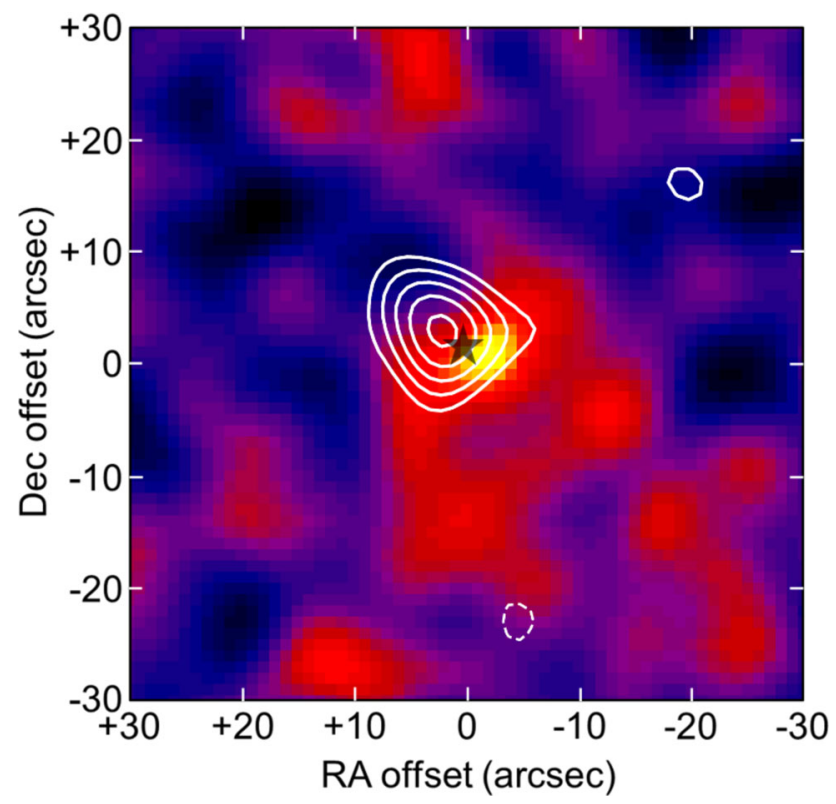

Figure 9. The $450 \mu \mathrm{m}$ image from observations of $\tau$ Ceti (HD 10700) with contours from the $850 \mu \mathrm{m}$ image overlaid. The contours and symbols are as described in Fig. 7

a $\beta$ constrained between 0 and 1.1, and implying a dust mass of $2.1 \times 10^{-4} \mathrm{M}_{\oplus}$ surrounding the star, only approximately 20 times that of the Edgeworth-Kuiper belt (Greaves et al. 2004).

\subsection{Hd 10638}

HD 10638 is a luminous A3 star at a distance of 69 pc with an estimated age of $50 \mathrm{Myr}$, with lower and upper limits of 20 and $150 \mathrm{Myr}$ (Moór et al. 2006). Although the SONS survey image is somewhat uneven (i.e. there is a prominent gradient in the background towards the west), an emission peak is detected at $850 \mu \mathrm{m}$ with a flux density of $5.1 \pm 0.9 \mathrm{mJy}$ (Fig. A2c) that is centred on the stellar position. As an unresolved disc about the star the upper limit to the radius is $515 \mathrm{au}$.

IRAS detected excess far-IR emission from the star (Silverstone 2000), but no other far-IR photometric measurements have been made. The SED is therefore loosely constrained by only the IRAS $60 \mu \mathrm{m}$ and SONS $850 \mu \mathrm{m}$ photometry, but the fit suggests a dominant cold component at $34 \mathrm{~K}$ but with a large uncertainty, a $\beta$ value of $>0.6$, and an estimated dust mass of $0.18 \mathrm{M}_{\oplus}$. Modelling of the near-mid IR photometric points of the SED also hints at the presence of a warmer, inner component at $138 \mathrm{~K}$.

\section{$5.8 \beta$ Trianguli (HD 13161)}

HD 13161 ( $\beta$ Tri) is a spectroscopic binary (primary of spectral type A5-IV) at a distance of $39 \mathrm{pc}$, with a period of 31 days. It has an estimated age of $730 \mathrm{Myr}$ with an uncertainty of $\pm 300 \mathrm{Myr}$ (Vican 2012). The $850 \mu \mathrm{m}$ image shows a peak in emission at the stellar position with a flux density of $5.1 \pm 0.9 \mathrm{mJy}$ (Fig. A2d). Interpreting this unresolved peak to be a disc about the star gives an upper limit to the radius of $290 \mathrm{au}$.

The disc is well-characterized in the far-IR, particularly with Herschel contributing to points in the SED. Herschel/PACS observations at $70 \mu \mathrm{m}$ reveal a deconvolved radius of $102 \mathrm{au}$ at a PA of $66^{\circ}$ (Booth et al. 2013). Modelling of the Herschel images 
concluded that the disc is consistent with being aligned with the binary orbital plane (Kennedy et al. 2012). The SED is modelled as a single modified blackbody with a characteristic dust temperature of $84 \mathrm{~K}$ and a $\beta$ in the range $0.6-1.7$. The estimated dust mass is $0.022 \mathrm{M}_{\oplus}$.

\section{$5.9 \gamma$ Trianguli (HD 14055)}

HD 14055 ( $\gamma$ Tri) has a spectral type A1 and is at a distance of $34 \mathrm{pc}$. It has an estimated age of $230 \mathrm{Myr}$ with a lower and upper limits of $160 \mathrm{Myr}$ (Thureau et al. 2014) and $300 \mathrm{Myr}$ (Chen et al. 2014), respectively. The $850 \mu \mathrm{m}$ image shows an emission morphology that peaks at an offset of 3 arcsec from the star, and appears to be slightly elongated in the north-south direction. The peak flux is $7.2 \pm 1.0 \mathrm{mJy}$ beam $^{-1}$ (Fig. A3a), a value slightly higher than previously reported (Panić et al. 2013). The integrated flux density, determined over a 40 arcsec diameter aperture, however, reveals no significant evidence of extended emission compared to the beam. The flux is also consistent with that measured using SCUBA of $5.5 \pm 1.8 \mathrm{mJy}$ (Williams \& Andrews 2006). There is no SCUBA-2 detection at $450 \mu \mathrm{m}$ with the $5 \sigma$ upper limit of $70 \mathrm{mJy}$ being close to the predicted flux from the SED. Interpreting this structure as a disc about the star gives an upper limit to the disc radius from the $850 \mu \mathrm{m}$ image of $260 \mathrm{au}$.

The disc has been resolved by Herschel/PACS observations at 70, 100 and $160 \mu \mathrm{m}$ and interpreted as a fairly broad disc extending from a radius of 3.7-5 $\operatorname{arcsec}(\sim 125-170 \mathrm{au})$, at a PA of $163^{\circ}$ from the $160 \mu \mathrm{m}$ image (Booth et al. 2013). Based on the Herschel results the north-south extension hinted at in the SONS $850 \mu \mathrm{m}$ image could be real, although, as previously mentioned, the integrated flux measurement indicates that it is not statistically significant. The $850 \mu \mathrm{m}$ SONS survey photometry helps to anchor the longwavelength side of the SED, allowing a single blackbody fit with a dust temperature of $77 \mathrm{~K}$, a $\beta$ emissivity value of between 0.7 and 1.5 , and an estimated dust mass of $0.028 \mathrm{M}_{\oplus}$.

\subsection{Hd 15115}

HD 15115 is a young F2 star within the $\beta$ Pictoris Moving Group at a distance of $45 \mathrm{pc}$, with an estimated age of $23 \pm 3 \mathrm{Myr}$ (Mamajek $\&$ Bell 2014). The $850 \mu \mathrm{m}$ image shows a structure with a slightly elongated morphology (PA of $\sim 150^{\circ}$ ), with a peak in emission 5 arcsec offset to the north from the star position (Fig. A3b). The flux density of $8.2 \pm 1.1 \mathrm{mJy}$ is marginally lower than that previously reported (Panić et al. 2013). The elongation is not significant compared to the beam size, and so interpreting the structure as an unresolved disc at the star gives an upper limit to the radius of $340 \mathrm{au}$.

HD 15115 has been observed in optical scattered light with images showing a remarkable asymmetry between the eastern and western sides of the disc (Kalas et al. 2007), with the western side reaching a radius of $12 \operatorname{arcsec}(\sim 550 \mathrm{au})$ from the star. The PA of the major axis of the disc from the scattered light image is $98.5^{\circ}$. Observations with the SMA at $1.3 \mathrm{~mm}$ resolved the HD 15115 disc into an asymmetric structure extending to the west of the star with a measured radius of 6 arcsec (MacGregor et al. 2015). Modelling of the image suggests a well-defined emission belt at a radius of 110 au from the star, and an $\sim 3 \sigma$ feature aligned with the asymmetric western extension of the scattered light disc (MacGregor et al. 2015). A fit to the Herschel/PACS, $850 \mu \mathrm{m}$ and $6.8 \mathrm{~mm}$ photometry from ACTA (MacGregor et al. 2016a) suggests a dominant cold

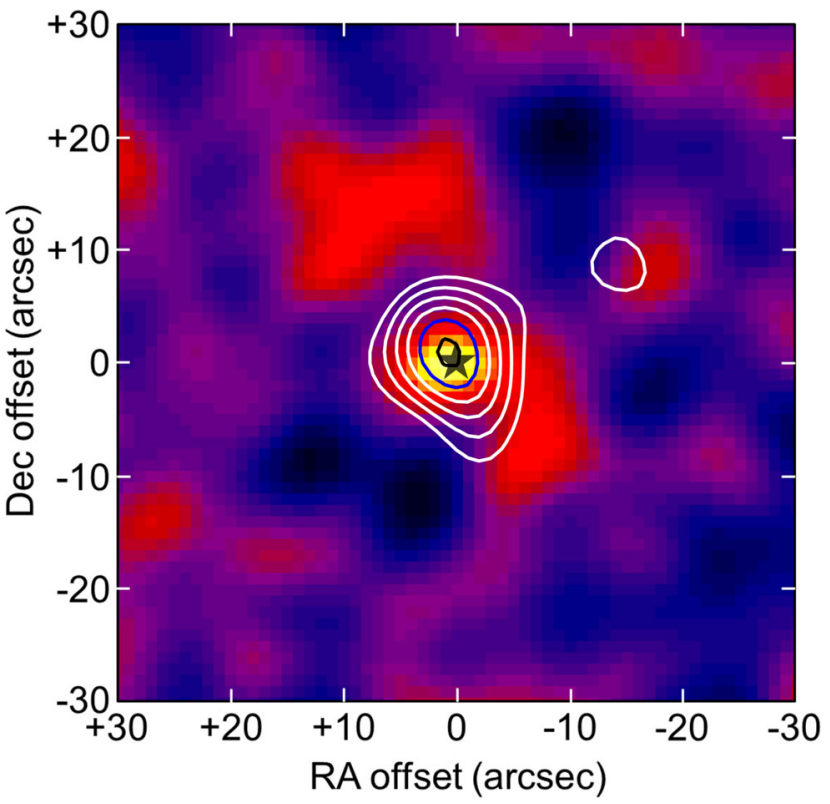

Figure 10. The $450 \mu \mathrm{m}$ image from observations of HD 15257 with contours from the $850 \mu \mathrm{m}$ image overlaid. The contours and symbols are as described in Fig. 7.

disc of characteristic temperature $57 \mathrm{~K}$, a $\beta$ in the range $0.5-0.9$, and an estimated dust mass of $0.073 \mathrm{M}_{\oplus}$.

\subsection{Trianguli (HD 15257)}

HD 15257 (12 Tri) is an F0 star at a distance of 50 pc, with an estimated age of $1000 \mathrm{Myr}$ (Chen et al. 2014). The $850 \mu \mathrm{m}$ image reveals a well-detected emission peak with a flux density of $10.3 \pm$ $1.2 \mathrm{mJy}$ (Fig. A3c) well-centred on the star position. Emission is also detected at $450 \mu \mathrm{m}$ in the SONS survey with a flux of $56 \pm$ $11 \mathrm{mJy}(\sim 5.1 \sigma)$ as shown in Fig. 10. The emission is unresolved at both wavelengths, and interpreting the results as being a disc about the star gives an upper limit to the disc radius from the $450 \mu \mathrm{m}$ image of 240 au.

IRAS detected excess far-IR emission from the star at $60 \mu \mathrm{m}$, but no other far-IR photometric measurements have been made. The SED is therefore sparsely sampled by only the IRAS and SONS 450 and $850 \mu \mathrm{m}$ photometry leading to a poorly constrained value of $\beta$ in the range $0-1.9$. The SED is modelled by a two-component fit, the warm element of which has a dust temperature $161 \mathrm{~K}$, based on a fit to the near/mid-IR photometric points from AKARI (Ishihara et al. 2010), Spitzer (Chen et al. 2014), and WISE (Wright et al. 2010). The dominant cold component is at $53 \mathrm{~K}$, and the disc has an estimated dust mass of $0.11 \mathrm{M}_{\oplus}$.

\subsection{Hd 15745}

HD 15745 is a young F2 star also in the $\beta$ Pictoris Moving Group, lying at a distance of $64 \mathrm{pc}$ with an estimated age of $23 \mathrm{Myr}$ (Mamajek \& Bell 2014). The $850 \mu \mathrm{m}$ image shows a well-detected, elongated structure with a peak flux of $8.4 \pm 1.0 \mathrm{mJy} \mathrm{beam}^{-1}$ (Fig. A3d). The peak is offset to the south from the stellar position by $\sim 3$ arcsec. Even though the star is at $64 \mathrm{pc}$, the structure appears to be marginally resolved with an integrated flux, as measured in a 50 arcsec diameter aperture centred on the star, of $12.0 \pm 1.4 \mathrm{mJy}$. The fitted radial extent of the structure gives a deconvolved radius 


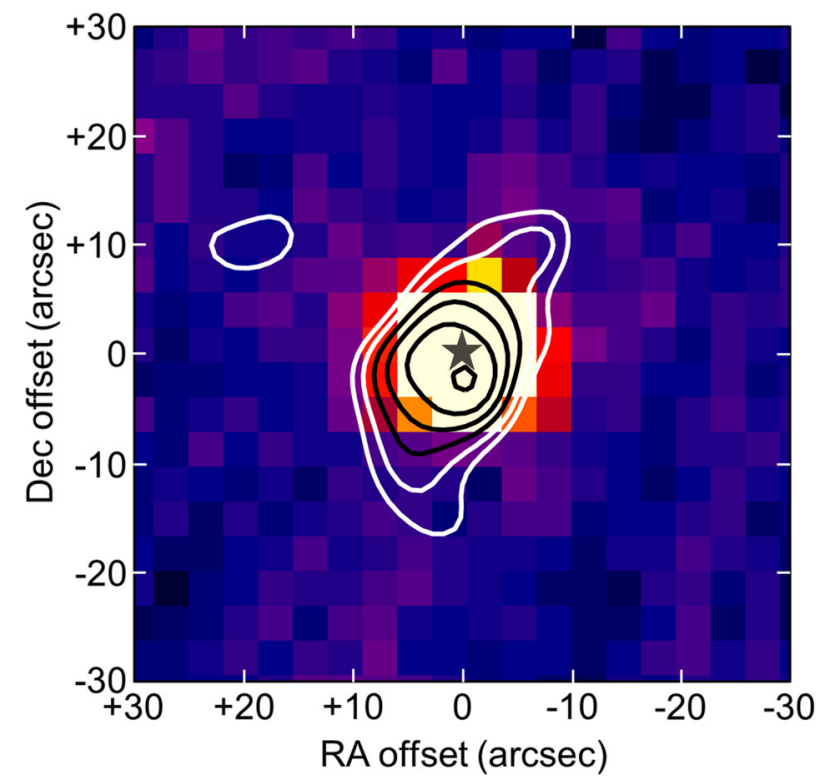

Figure 11. Herschel/PACS $160 \mu \mathrm{m}$ image of HD 15745 with contours from the SONS survey $850 \mu \mathrm{m}$ image overlaid. The contours are $3 \sigma$ and $4 \sigma$ (white) and then $5 \sigma-8 \sigma$ (black). The star symbol represents the position of the star with respect to the flux peak. The Herschel image is taken from the Herschel Science Archive.

of $8.1 \operatorname{arcsec}(\sim 510 \mathrm{au})$, suggesting the existence of a very large disc about the star at a PA of $164^{\circ}$

In optical scattered light observations using the HST/ACS coronograph, the disc appears asymmetric about the star at a PA of $\sim 22.5^{\circ}$, with fan-shaped emission extending to $480 \mathrm{au}$ in the region between PAs of 190 and $10^{\circ}$ (Kalas et al. 2007b; Schneider et al. 2014). The scattered light emission seems to extend to a radius close to that seen in the SONS $850 \mu \mathrm{m}$ image $(\sim 8 \operatorname{arcsec} ; \sim 510$ au) but the orientation of the disc in the submillimetre does not show the same asymmetry as in scattered light. Herschel/PACS images at 70 and $160 \mu \mathrm{m}$ show that the disc appears to be well-centred on the stellar position but there is little evidence of the extension seen in the $850 \mu \mathrm{m}$ image (as shown in Fig. 11).

The SED is reasonably well-sampled in the mid-far-IR from IRAS (Rhee et al. 2007), Spitzer/MIPS at $70 \mu \mathrm{m}$ (Moór et al. 2011b), and Herschel/PACS (archival data) leading to a constraint on $\beta$ between 0 and 0.9 . The model fit through the photometric points yields a dust temperature of $89 \mathrm{~K}$, leading to an estimated dust mass of $0.13 \mathrm{M}_{\oplus}$. The relatively high derived dust temperature means that the modelled disc radius, assuming that the grains behave as blackbodies, lies close to the star at only 18 au. Hence, there is a large discrepancy (a factor of 28) in the modelled disc radius and that measured from the resolved image. This is further discussed in Section 6.2.

\subsection{Arietis (HD 17093)}

HD 17093 (38 Ari) is a luminous A7 star at a distance of $36 \mathrm{pc}$ with an estimated age of $580 \mathrm{Myr}$ (Vican 2012). The SONS survey $850 \mu \mathrm{m}$ image was first reported by Panić et al. (2013) and shows a well-detected peak of flux density $8.8 \pm 0.9 \mathrm{mJy}$, which is offset from the stellar position by $10 \operatorname{arcsec}$ (Fig. A4a). This offset is far in excess of what would be expected for a disc about the star, allowing for positional and statistical uncertainties in the measurement (see Section 6.4). It is therefore likely that the peak is due to a separate source, perhaps a background galaxy. This hypothesis is further supported by Herschel/PACS archival images showing significant 100 and $160 \mu \mathrm{m}$ emission at the same offset position to the SCUBA-2 $850 \mu \mathrm{m}$ map (Panić et al. 2013). A fit to the far-IR photometric and submillimetre points (Fig. A4a) suggests an object with a very cold $(\sim 14 \mathrm{~K})$ dust temperature. The conclusion is that there is no current evidence for a debris disc surrounding HD 17093.

\subsection{Algol (HD 19356, $\beta$ Persei)}

HD 19356 (Algol) is a triple-star system with the primary of spectral type B8-V, lying at a distance of $28 \mathrm{pc}$ with an age estimated at $450 \mathrm{Myr}$, although it could be as young as $300 \mathrm{Myr}$ (Rhee et al. 2007) or as old as $570 \mathrm{Myr}$ (Söderhjelm et al. 1980). The bright primary (Algol/ $\beta$ Per Aa1) is eclipsed by the fainter secondary (Aa2; separation of $0.062 \mathrm{au}$ ) on a period of $2.9 \mathrm{~d}$. The third star in the system $(\mathrm{Ab})$ lies at a distance of 2.7 au from the primary. The SONS survey $850 \mu \mathrm{m}$ image shows well-detected emission with a peak flux $6.4 \pm 0.9 \mathrm{mJy}$ beam $^{-1}$, centred on the stellar position (Fig. A4b). Although the morphology of the emission looks somewhat extended at $850 \mu \mathrm{m}$, the integrated flux, determined in a 30 arcsec diameter aperture, is in good agreement to the peak value, suggesting the structure is unresolved. Given that all three stars lie within an area subtended by the telescope beam, it is impossible to ascertain which star is responsible for the excess flux at submillimetre wavelengths.

Although Spitzer/MIPS detected emission from Algol at 24 and $70 \mu \mathrm{m}$ (Su et al. 2006), a modified blackbody fit to these points and the new SONS survey $850 \mu \mathrm{m}$ flux is not possible. Multiple discs may be present, with an ultra-cold component dominating the emission at $850 \mu \mathrm{m}$. The SED model fit through the $850 \mu \mathrm{m}$ point (Fig. A4b) is only indicative, as there are no other constraining points. Indeed, a cold disc component with a temperature of around $27 \mathrm{~K}$ could exist (noting a large uncertainty of $\pm 17 \mathrm{~K}$ in this temperature estimation). The estimated dust mass based on the $850 \mu \mathrm{m}$ flux density is $0.045 \mathrm{M}_{\oplus}$. Algol Aa1 is $\sim 100$ times more luminous than Sun. Combined with a relatively low derived dust temperature, this high luminosity suggests the radius of a disc (assuming the grains behave as blackbodies) could be as large as $\sim 1100$ au (again, with large uncertainties). This value compares to only 210 au based on the upper limit to the disc radius from the $850 \mu \mathrm{m}$ image.

The emission from the Algol system is known to be highly variable and of very high brightness temperature $\left(\sim 10^{9} \mathrm{~K}\right)$ (Lestrade et al. 1988). More specifically, it has been identified as gyrosynchrotron emission from mildly relativistic electrons accelerated by magnetic reconnections in its stellar chromosphere (as also known for Sun and other stars). The spectrum of such emission peaks at a few centimetres, and may extend into the millimetre wavelength domain (Dulk 1985). The flux observed at $850 \mu \mathrm{m}$ therefore might be such a tail, rather than thermal emission from a debris disc. The recently measured variability of the millimetre flux density observed at the SMA strengthens this interpretation (Wyatt/Wilner, private communication).

There are, however, also a number of possible scenarios to explain the presence of cool debris around the star. First, a dust ring may surround at least one of the stars but companion perturbations in the system may cause it to be disrupted, hampering the formation of planets but allowing for the formation of enough planetesimals to generate significant debris material (e.g. Thebault \& Haghighipour 2015). Second, a significant part of the emission may be related to mass loss from the secondary (and/or tertiary) to the primary, resulting in the presence of circumstellar material (Miller et al. 2006). Finally, there could be a mass outflow from the mass-gaining star, so the 
system loses angular momentum (the 'non-conservative' problem) with the outflow, which then forms a shock as it encounters the interstellar medium (Deschamps et al. 2015). High angular resolution observations are needed to resolve the proposed structures and give further insight on the true nature of any 'debris' surrounding Algol. Hence, although it may be possible for a debris disc to exist around Algol, evidence from longer wavelengths points to the emission at $850 \mu \mathrm{m}$ being due to radio variability rather than a debris disc.

\subsection{Hd 21997}

HD 21997 is a young A3 star in the Columba Moving Group, at a distance of $72 \mathrm{pc}$ and an estimated age of $30 \mathrm{Myr}$ (Torres et al. 2008) with an uncertainty for the group in the range 20-50 Myr (Marois et al. 2010). At $850 \mu \mathrm{m}$, the emission appears slightly elongated to the south at a PA of $25^{\circ}$ with a peak flux of $7.9 \pm 1.1 \mathrm{mJy} \mathrm{beam}^{-1}$ at the stellar position (Fig. A4c). The peak flux is also consistent with that measured using SCUBA photometry of $8.3 \pm 2.3 \mathrm{mJy}$ (Williams \& Andrews 2006). The integrated flux density, measured over a 40 arcsec diameter aperture, is $10.7 \pm 1.5 \mathrm{mJy}$, suggesting that, even at a distance of $72 \mathrm{pc}$, the structure is marginally resolved. This extent, however, depends largely on the significance of the feature to the south within the $3 \sigma$ contour. The fitted radial extent taking in this extension suggests that HD 21997 could be surrounded by an enormous disc of radius 810 au at a PA of $27^{\circ}$.

Herschel/PACS and SPIRE observations resolved the disc, identifying a structure with a radius of at least $2.8 \operatorname{arcsec}(\sim 200 \mathrm{au})$ at a PA of $\sim 25^{\circ}$ (Moór et al. 2015). The disc has also been resolved by ALMA at $886 \mu \mathrm{m}$ revealing an inclined ring-like structure of radius $\sim 2.1 \operatorname{arcsec}(\sim 150 \mathrm{au})$ at a PA of $22.5^{\circ}$ (Moór et al. 2013). Modelling of the morphology of the ring suggests inner and outer radii of $\sim 55$ and $\sim 150$ au, respectively. The measured flux from the ALMA image is $2.7 \pm 0.3 \mathrm{mJy}$, considerably lower than previously measured at $850 \mu \mathrm{m}$, suggesting that some of the emission has been resolved out by the interferometric observations, and leading to a significant underestimate of the amount of dust present in the system. Such a hypothesis would be consistent with detectable emission extending beyond the outer radius of $\sim 150$ au derived from ALMA, as indicated in the Herschel and $850 \mu \mathrm{m}$ SONS observations (perhaps extending to $\sim 500$ au or more).

HD 21997 is also one of the few systems with an age greater than $10 \mathrm{Myr}$ that contains a detectable amount of cold CO gas (Moór et al. 2011a). The SED contains photometric points in the mid-far-IR from Spitzer/MIPS and Herschel/PACS and SPIRE. The well-constrained fit through the photometric points gives a dust temperature of $64 \mathrm{~K}$, and a $\beta$ of between 0.4 and 1.4. The estimated dust mass of $0.22 \mathrm{M}_{\oplus}$ is considerably higher than the value of $0.09 \mathrm{M}_{\oplus}$, reported based on the ALMA observations, due to the aforementioned larger flux.

\section{$5.16 \epsilon$ Eridani (HD 22049)}

$\epsilon$ Eridani (HD 22049) is a nearby K2 star at a distance of $3.2 \mathrm{pc}$ with an estimated age of $850 \mathrm{Myr}$ within a range spanning $800 \mathrm{Myr}$ (Di Folco et al. 2004; Mamajek \& Hillenband 2008) and 1.4 Gyr (Bonfanti et al. 2015). The SONS image at $850 \mu \mathrm{m}$ reveals a wellresolved, largely circularly symmetric, ring structure with considerably less emission at the stellar position (Fig. A4d). There appears to be significant sub-structure in the ring with the brightest clump to the south-east having a flux of $5.3 \pm 0.7 \mathrm{mJy} \mathrm{beam}^{-1}$. The integrated flux, measured within a 70 arcsec diameter aperture centred on the star, is $31.3 \pm 1.9 \mathrm{mJy}$. Both the peak clump flux and the total

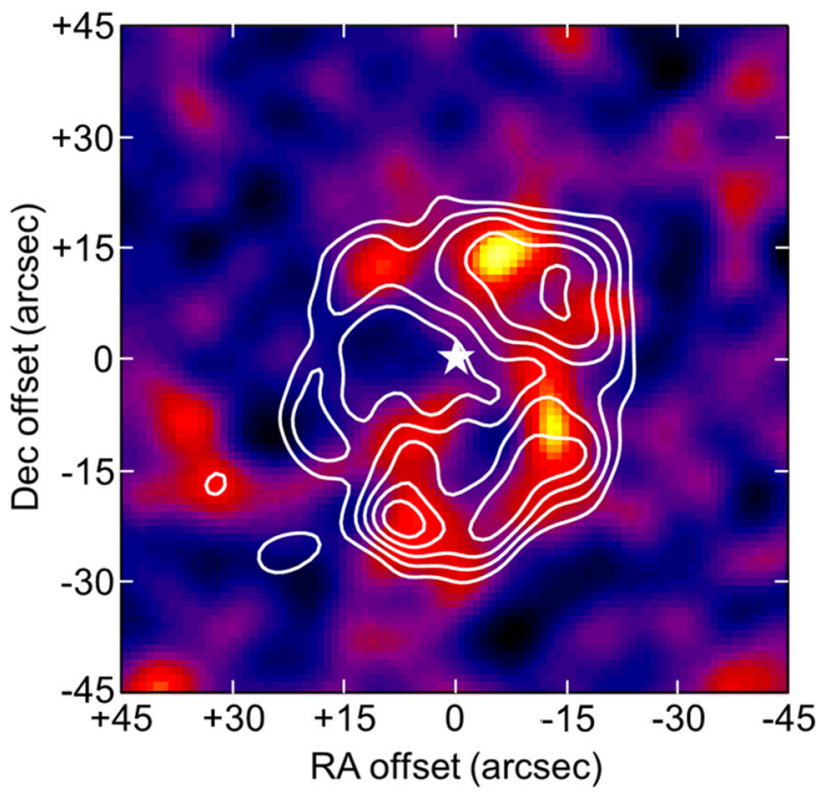

Figure 12. The $450 \mu \mathrm{m}$ image from observations of $\epsilon$ Eridani (HD 22049) with contours from the $850 \mu \mathrm{m}$ image overlaid. The contours and symbols are as described in Fig. 7.

integrated flux agree well with the previous SCUBA observations (Greaves et al. 1998, 2005), although the latter is some 15 per cent lower, but within the measured uncertainties. Interpreting the structure as a dust ring about the star gives a deconvolved radius, based on the Gaussian fit to the $850 \mu \mathrm{m}$ image, of $20.8 \operatorname{arcsec}(\sim 67 \mathrm{au})$. The inclination angle to the plane of the sky is estimated to be $26^{\circ}$, also consistent with the SCUBA results. The image at $450 \mu \mathrm{m}$ only shows clumps with low $\mathrm{S} / \mathrm{N}(\sim 3-5 \sigma)$, but nevertheless suggests a ring structure that is not inconsistent with the results at $850 \mu \mathrm{m}$ (Fig. 12). The integrated flux, within a 70 arcsec diameter aperture, is $181 \pm 15 \mathrm{mJy}$, which compares to $250 \pm 20 \mathrm{mJy}$ determined by Greaves et al. (2005). Significantly more integration time is ideally needed to improve upon the $450 \mu \mathrm{m}$ results.

Given its age and close proximity to Sun in both distance and spectral type, the $\epsilon$ Eridani dust ring has been widely studied at multiple wavelengths. The original $850 \mu \mathrm{m}$ SCUBA image from 1998 first revealed the ring-like structure, peaking at a radius of $60 \mathrm{au}$ from the star and with an estimated dust mass of at least $0.01 \mathrm{M}_{\oplus}$ (Greaves et al. 1998). It was concluded that the ring structure was probably akin to the Edgeworth-Kuiper belt in our Solar system with a central region partially cleared of dust by forming planetesimals. Further deep imaging with SCUBA over the period 1999-2004 confirmed the dust ring morphology as well as producing the first image of the structure at $450 \mu \mathrm{m}$ (Greaves et al. 2005). The latter work also presented tentative evidence that two clumps in the ring were following the stellar motion (i.e. were not background objects) and furthermore showed evidence of counter-clockwise rotation. It was suggested that the structure in the ring could be caused by perturbations of a planet orbiting the star at a few tens of $\mathrm{AU}$, and led to the speculation that the $\epsilon$ Eridani system could resemble an early version of our Solar system.

Far-IR observations with Spitzer indicated the likely presence of two unresolved warm, inner dust rings, in addition to a wider, icy outer belt (Backman et al. 2009). The inner belt lies at a radius of around $3 \mathrm{au}$, very similar to the distance of the Asteroid belt in the Solar system. There is also at least one candidate Jupitermass planet ( $\epsilon$ Eri b) in the system believed to lie just outside 
of this belt at an orbital radius of $3.5 \mathrm{au}$, discovered using the radial-velocity (RV) technique (Hatzes et al. 2000). Astrometric observations also provide evidence for the existence of a planet and allow its inclination to be determined (Benedict et al. 2006; Reffert \& Quirrenbach 2011). Although the planet's orbit appears to be inclined at a similar angle $\left(\sim 30^{\circ}\right)$ to the disc, the inclination is in a very different direction, meaning that the disc and planetary orbit are not likely coplanar. The planet has also proven somewhat controversial as extensive followup RV observations have so far failed to confirm its existence (Zechmeister et al. 2011). Herschel observations at 160-350 $\mu \mathrm{m}$ resolved two belts of debris emission. The inner belt has a radius of 12-16 au, whilst the outer cold belt orbited the star at a radius of 54-68 au (Greaves et al. 2014). There has also been considerable speculation about the existence of a planet orbiting at a distance close to the outer dust belt. The brightness asymmetry between the north and south ends of the belt from the Herschel observations indicates a pericentre glow that could be attributable to such a planet.

The clumpy structure of the observed disc was presented as evidence that dynamical interaction between an unseen planetary companion and the debris belt (Quillen \& Thorndike 2002). Millimetrewave observations with MAMBO-2 on the IRAM $30 \mathrm{~m}$ telescope at $1.2 \mathrm{~mm}$ also confirmed the clumpy structure seen in the submillimetre images (Lestrade \& Thilliez 2015). However, other observations, most notably millimetre-wave observations using the bolometer array SIMBA on SEST (Schütz et al. 2004) and the SMA interferometer (MacGregor et al. 2015), did not confirm the presence of significant substructure within the ring. More recently, the AzTEC camera on the $50 \mathrm{~m}$ Large Millimeter Telescope imaged the $\epsilon$ Eridani dust belt reaching an RMS noise level of $0.12 \mathrm{mJy}$ at $1.1 \mathrm{~mm}$ (Chavez-Dagostino et al. 2016). The measured radius for the outer belt of 64 au agrees well with other measurements, and although there are some inhomogeneities, the observed structure indicates a morphology that is essentially smooth. The deep LMT image also highlights the presence of numerous point-like sources, with the likelihood, based on the near 20 years of observations (and noting the large stellar proper motion of $1 \operatorname{arcsec} \mathrm{yr}^{-1}$ ), that at least one of the original submillimetre clumps within the ring is likely to be a background source. The northern arc of the ring has also been imaged by ALMA at a wavelength of $1.3 \mathrm{~mm}$, revealing tentative evidence of the presence of a clumpy structure in the ring (Booth et al. 2017). The ring is very narrow at just 11-13 au, making the fractional disc width comparable with the Edgeworth-Kuiper belt in our Solar system.

The SED is well-sampled in the far-IR through to the millimetre. There is somewhat of a discrepancy in the total disc flux between the recent millimetre-wave observations (IRAM/MAMBO-2 and the LMT/AzTec) and a fit to the Spitzer, Herschel, SCUBA, SCUBA-2 and the original IRAM photometry as shown in the SED in Fig. A4d. The reason for this is unclear, but may be very dependent on the precision of the aperture photometry with respect to the ring and the presence of background sources. Modelling of the SED suggests a two-component fit to the overall IR excess consistent with the results from Spitzer and the resolved imaging from Herschel. The fit to the warmer, inner component suggests the existence of an 'asteroid belt' at an orbital radius of $\sim 14$ au with a characteristic temperature of $133 \mathrm{~K}$. The colder component is the more familiar outer ring from the submillimetre observations at a radius of $\sim 60$ au, modelled as a disc/belt with a dust temperature $44 \mathrm{~K}$, and a $\beta$ value in the range of 0.6-1.0, with an estimated dust mass of only $0.002 \mathrm{M}_{\oplus}$ calculated from the $850 \mu \mathrm{m}$ flux. The derived dust temperature for the cold disc is also in good agreement with previous estimates
(Greaves et al. 1998, 2005). The equivalent disc radius, assuming blackbody emission, is 23 au, this being significantly lower than the value measured from the $850 \mu \mathrm{m}$ image of $67 \mathrm{au}$.

\subsection{Hd 22179}

HD 22179 is a young G5 star, at a distance of $16 \mathrm{pc}$ and an estimated age of only $16 \mathrm{Myr}$ (Carpenter et al. 2009). At $850 \mu \mathrm{m}$, there is a bright emission peak with a flux density of $7.0 \pm 1.3 \mathrm{mJy}$, located 18 arcsec from the stellar position to the south west (Fig. A5a). Given the large offset, it is unlikely that the peak is associated with a putative disc around the star, and is probably a background object. At far-IR wavelengths Spitzer detected excess flux from the star at $70 \mu \mathrm{m}$ (just over $3 \sigma$ ), but no detection was made in the longer wavelength $160 \mu \mathrm{m}$ channel (Hillenbrand et al. 2008). Photometry shortward of $24 \mu \mathrm{m}$ indicated clear evidence of a flux excess, but it is also possible that Spitzer detected the same offset peak seen in the SONS $850 \mu \mathrm{m}$ image. Hence, there is some uncertainty as to whether a debris disc does exist around HD 22179.

\subsection{Hd 25457}

HD 25457 is a F6 star in the AB Doradus Moving Group, at a distance of $19 \mathrm{pc}$ with a somewhat controversial age but generally accepted to be very similar to the Pleiades at $130 \pm 20$ Myr (Barrado y Navascués, Stauffer \& Jayawardhana 2004). The SONS image at $850 \mu \mathrm{m}$ shows an unresolved peak of flux density $6.4 \pm 1.4 \mathrm{mJy}$, offset from the stellar position by 2 arcsec (Fig. A5b). Interpreting the peak as an unresolved disc associated with the star gives an upper limit for the disc radius of $140 \mathrm{au}$. The $850 \mu \mathrm{m}$ image shows some unevenness, with ridges of excess flux towards the east and corresponding negative features in the west.

Spitzer detected excess flux from the star at wavelengths between 3.6 and $160 \mu \mathrm{m}$ (Hillenbrand et al. 2008). Although there have been several observations of the star in the submillimetre and millimetre, the SONS $850 \mu \mathrm{m}$ image is the only clear detection of a disc at such long wavelengths. Modelling of the SED suggests a two-component fit to the overall IR excess consistent with the results from Spitzer and the imaging from SONS. The fit to the warmer component, dominated by the near-mid IR photometry, gives a characteristic temperature of $124 \mathrm{~K}$. The colder component has a dust temperature of $50 \mathrm{~K}$, a $\beta$ value only loosely defined as $>0.1$, and an estimated dust mass of $0.011 \mathrm{M}_{\oplus}$ derived from the $850 \mu \mathrm{m}$ flux.

\subsection{Hd 35841}

HD 35841 is a young F3 star, also in the Columba Moving Group, at a distance of $96 \mathrm{pc}$ with an estimated age of $30 \mathrm{Myr}$. The SONS survey image at $850 \mu \mathrm{m}$ reveals several peaks in the vicinity of the star, including a faint $4 \sigma$ peak offset from the star by $\sim 5 \operatorname{arcsec}$ to the south, with a flux density of $3.5 \pm 0.8 \mathrm{mJy}$ (Fig. A5c). To the south-west, some 23 arcsec offset, is another peak with a flux of $3.1 \pm 0.8 \mathrm{mJy}$ and a low-level $(\sim 2.5 \sigma)$ ridge running between the two that extends to the north-east of the star. If the peak just to the south is an unresolved disc associated with the star, then the upper limit for the disc radius is $725 \mathrm{au}$.

The disc has been detected in scattered light via HST/NICMOS observations that show a very compact, nearly edge-on disc with a radius of $1.5 \operatorname{arcsec}(\sim 144 \mathrm{au})$ from the star (Soummer et al. 2014). The two lobes are not diametrically aligned with PAs of $\sim 180$ and $\sim 335^{\circ}$. Only Spitzer/MIPS photometry at 24 and $70 \mu \mathrm{m}$ (Chen et al. 2014) contributes to an otherwise poorly constrained SED. A fit to 
the SED, assuming the peak detected at $850 \mu \mathrm{m}$, is representative of a disc about the star, gives a dust temperature of $71 \mathrm{~K}$, a $\beta$ value very loosely constrained in the range $0-2.7$, and an estimated dust mass of $0.11 \mathrm{M}_{\oplus}$.

\subsection{Hd 38858}

HD 38858 is a nearby ( 15 pc) Sun-like star of spectral type G4, hosting a super-Earth planet within $1 \mathrm{AU}$, with a highly uncertain stellar age usually adopted as being $4700 \mathrm{Myr}$ (Beichman et al. 2006), but likely to be in the range of $3200 \mathrm{Myr}$ (Chen et al. 2014) to $6200 \mathrm{Myr}$ (Vican 2012). The SONS observation at $850 \mu \mathrm{m}$ (Fig. A5d) reveals a large extended structure with an integrated flux density, measured in a 60 arcsec diameter aperture, of $11.5 \pm 1.3 \mathrm{mJy}$ (Kennedy et al. 2015). In terms of the interpretation of the image, the peak is clearly offset from the star position by 9 arcsec to the east, although there is still significant emission at the star position itself $\left(\sim 5 \mathrm{mJy} \mathrm{beam}^{-1}\right)$. One possibility is that the extended structure to the south-east is a separate background source.

Herschel/PACS observations at $70 \mu \mathrm{m}$ reveal a disc of radius 7.5 $\operatorname{arcsec}(\sim 110 \mathrm{au})$ at a PA of $67^{\circ}$, which is well-centred at the stellar position (Kennedy et al. 2015), and similar to the results reported by Spitzer/MIPS (Krist et al. 2012). Observations at $160 \mu \mathrm{m}$, however, reveal a separate source to the south, coincident with the peak flux of the $850 \mu \mathrm{m}$ extension. Hence, there is a strong likelihood that this source is a background object (perhaps a high redshift galaxy given the absence of such a source at $70 \mu \mathrm{m}$ ), and therefore the remainder of the discussion on this target assumes this to be the case. Finally, the image is also likely 'contaminated' at some level by the Orion Complex background emission to the east of the star (Kennedy et al. 2015).

By constructing models of the HD 38858 disc, it is possible to estimate the likely contribution to the true disc flux at $7.5 \pm 1.5 \mathrm{mJy}$, taking into account the background contamination and assuming the southern peak is not associated with a disc (Kennedy et al. 2015). This interpretation still assumes that the flux peak is at a distance of 9 arcsec from the star. With this single long wavelength point, the disc spectrum is now close to a pure blackbody with $\beta$ around zero. A fit to the SED gives a characteristic dust temperature of $50 \mathrm{~K}$ and an estimated dust mass of $0.0086 \mathrm{M}_{\oplus}$.

The radius of the disc, derived from the SED fit and assuming blackbody grain properties, is only 28 au. This value is significantly smaller than the measured deconvolved radius of the disc of $12.7 \operatorname{arcsec}(\sim 190 \mathrm{au})$ at a PA of $75^{\circ}$ (determined after subtracting a point-source at the location of the southern peak). Such a result is surprising given the requirement of a nearly pure blackbody spectrum fit to the SED (as described above), which would imply that the blackbody and resolved disc sizes should be in agreement, i.e. the disc material should lie at a single radius from the star. Blackbody spectra have been seen for other discs at larger radii than their blackbody temperatures would suggest. An example is AU Mic (Matthews et al. 2015), where it is likely that the submillimetre observations trace the parent-body distribution (which may have blackbody properties), whilst the far-IR data sample a halo of smaller grains from highly eccentric orbits due to radiation pressure. In summary, the SONS $850 \mu \mathrm{m}$ image of HD 38858 is interpreted as a resolved disc, with a peak flux offset from the star position by 9 arcsec and with the presence of a background object contaminating the observed structure to the south.

\subsection{Aurigae (HD 48682)}

HD 48682 (56 Aur) is an old F9 star, at a distance of 17 pc with an estimated age of around $6000 \mathrm{Myr}$ (Barry 1988), though could be as young as $3200 \mathrm{Myr}$ (Holmberg, Nordström \& Anderson 2009) or as old as $8900 \mathrm{Myr}$ (Beichman et al. 2006). The field at $850 \mu \mathrm{m}$ shows faint multiple peaks, of which the nearest to the star has a flux of $2.7 \pm 0.6 \mathrm{mJy}^{-1}$ beam $^{-1}$ (Fig. A6a). The peak is offset by 6.5 arcsec from the star, significantly greater than the maximum offset of 3.7 arcsec expected due to statistical and positional uncertainties (Section 6.4). The structure near the star could also be marginally extended compared to the beam, mainly in the east-west direction, and has an integrated flux density (in a 30 arcsec diameter aperture) of $3.9 \pm 0.8 \mathrm{mJy}$. Interpreting the structure nearest the star to be a disc, the radial fitting gives a deconvolved disc radius of 11 $\operatorname{arcsec}(\sim 184 \mathrm{au})$ at a PA of $94^{\circ}$, which is larger than the estimated radius of 57 au based on the assumption of pure blackbody emission from grains (see discussion in Section 6.3).

Observations with Herschel/PACS at $100 \mu \mathrm{m}$ reveal an elongated disc with a measured deconvolved radius $16.4 \operatorname{arcsec}(\sim 280 \mathrm{au})$ at a PA of $107^{\circ}$ (Eiroa et al. 2013; Pawellek et al. 2014) approximately 50 per cent larger than the estimate from the SONS $850 \mu \mathrm{m}$ image. The peak to the SW is not considered to be associated with this main disc. A fit to the SED, mainly sampled by Herschel photometry, and including the $850 \mu \mathrm{m}$ SONS flux, gives a dust temperature of $43 \mathrm{~K}$ and a $\beta$ in the range $0.7-1.8$, leading to an estimated dust mass of $0.0062 \mathrm{M}_{\oplus}$.

\subsection{HD 61005 ('The Moth')}

HD 61005 is a G8 star and a possible member of the Argus Association, at a distance of $35 \mathrm{pc}$ with an estimated age of $40 \mathrm{Myr}$ (Desidera et al. 2011), although other estimates include $100 \mathrm{Myr}$ (Hillenbrand et al. 2008) and in the range of 72-186 Myr (Desidera et al. 2011). The $850 \mu \mathrm{m}$ image shows emission peaking just offset from the stellar position and extending somewhat to the south-east, with a flux density of $13.5 \pm 2.0 \mathrm{mJy}$ (Fig. A6b). The elongation is not significant compared to the beam size, however, and interpreting the structure as a disc about the star gives an upper limit to the disc radius of 265 au.

The disc has been studied in detail at many wavelengths and results include the fan-like scattered light observation that led to the nickname of 'The Moth' (Hines et al. 2007). Observations with the SMA first resolved the disc at millimetre wavelengths, revealing a double-peaked structure, viewed close to edge-on, with a measured major axis radius of $2.2 \operatorname{arcsec}(\sim 80 \mathrm{au})$ at a PA of $71^{\circ}$ (Ricarte et al. 2013). ALMA observations at $1.3 \mathrm{~mm}$ also resolve the disc into a highly inclined $\left(i \sim 85^{\circ}\right)$ structure with the emission peaking at a radius of $1.5 \operatorname{arcsec}(\sim 55 \mathrm{au})$ from the star, with the disc having a PA of $71^{\circ}$ (Olofsson et al. 2016). Although the disc was not detected at $450 \mu \mathrm{m}$ in the SONS survey, the SED is further well-constrained in the mid-far-IR with photometry from both Spitzer/MIPS (Chen et al. 2014) and Herschel/PACS (Morales et al. 2016), and in the millimetre by photometry at $9 \mathrm{~mm}$ from the VLA (MacGregor et al. 2016a). The fit to the SED gives a characteristic dust temperature of $61 \mathrm{~K}$, and a $\beta$ value tightly constrained within the range $0.4-0.7$. The calculated dust mass from the $850 \mu \mathrm{m}$ flux is $0.069 \mathrm{M}_{\oplus}$.

\subsection{Gj 322}

GJ 322 is a K5 star in the Ursa Major Moving Group, at a distance of $16.5 \mathrm{pc}$ with an estimated age of $490 \pm 100 \mathrm{Myr}$ 


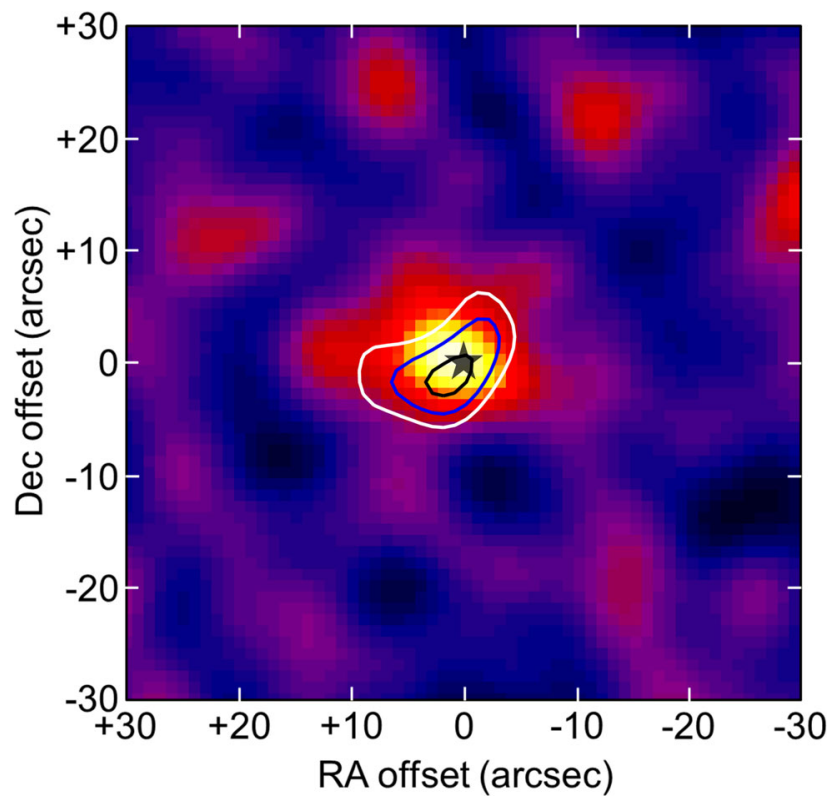

Figure 13. The $450 \mu \mathrm{m}$ image from observations of GJ 322 with contours from the $850 \mu \mathrm{m}$ image overlaid. The contours and symbols are as described in Fig. 7.

(Jones et al. 2015). The SONS survey results show an unresolved peak, well-centred on the stellar position, at both 450 and $850 \mu \mathrm{m}$ with flux densities of $57 \pm 11 \mathrm{mJy}$ and $7.3 \pm 1.4 \mathrm{mJy}$, respectively (Fig. A6c and Fig. 13). Interpreting the peak to be a disc about the star gives an upper limit to a disc radius of 85 au based on the $450 \mu \mathrm{m}$ image. The SED, however, is poorly constrained in the mid-far-IR with only Spitzer/MIPS photometry at $70 \mu \mathrm{m}$ (Chen et al. 2014), which, together with the steep spectral slope between 450 and $850 \mu \mathrm{m}$, leads to unconstrained values of both $\lambda_{0}$ and $\beta$. A fit to the SED gives a characteristic dust temperature of $24 \mathrm{~K}$ (but with a large error of $\pm 10 \mathrm{~K}$ ), and a derived dust mass of $0.021 \mathrm{M}_{\oplus}$. The disc radius from the SED fit is also poorly estimated at $43 \pm$ $24 \mathrm{au}$, assuming emission from pure blackbody grains.

\subsection{Cancri (HD 76582)}

HD 76582 (63 Cnc) is a moderately old F0 star, at a distance of $46 \mathrm{pc}$ with an estimated age of $540 \mathrm{Myr}$ (Moór et al. 2006) and a likely age in the range 300-2130 Myr (Zorec \& Royer 2002). The SONS survey observations show unresolved emission at both 450 and $850 \mu \mathrm{m}$ at the stellar position, with peak fluxes of $89 \pm 17$ and $5.7 \pm 1.0 \mathrm{mJy}$ beam $^{-1}$, respectively (Fig. A6d and Fig. 14). The $450 \mu \mathrm{m}$ flux estimate is based on a re-analyis of the data, and is slightly lower than previously published (Marshall et al. 2016). The $450 \mu \mathrm{m}$ image looks slightly extended in a roughly east-west direction, but aperture photometry reveals an integrated flux density that is not significant higher than the peak value. Based on the $450 \mu \mathrm{m}$ image, and interpreting the structure as a disc about the star, gives an upper limit to the radius of the disc of $230 \mathrm{au}$.

The disc was originally detected by IRAS and has been wellcharacterized in the mid-far-IR (Zuckerman \& Song 2004; Moór et al. 2006). The disc has also been resolved by Herschel/PACS, giving a radius of $5.9 \operatorname{arcsec}(\sim 271 \mathrm{au})$ at a PA of $103^{\circ}$ for the $100 \mu \mathrm{m}$ image, and $8.5 \operatorname{arcsec}(\sim 390 \mathrm{au})$ at a PA of $115^{\circ}$ at $160 \mu \mathrm{m}$ (Marshall et al. 2016). The disc is inclined at $64^{\circ}$ to the plane of the sky (Marshall et al. 2016). Based on these measurements, it

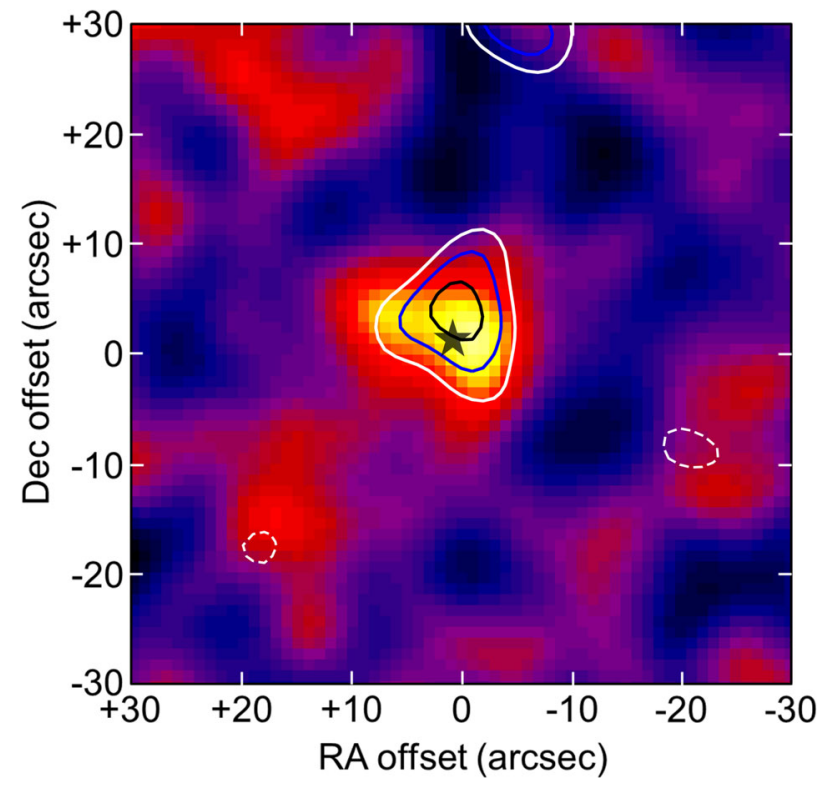

Figure 14. The $450 \mu \mathrm{m}$ image from observations of HD 76582 (63 Cnc) with contours from the $850 \mu \mathrm{m}$ image overlaid. The contours and symbols are as described in Fig. 7.

is possible that the disc might be marginally resolved at $450 \mu \mathrm{m}$ (even though the integrated flux suggests otherwise). At least a twocomponent, modified, blackbody model is needed to fit the photometric points from the near-IR to the submillimetre in the SED. The inner component, based on a fit to the near-mid IR photometry, has an estimated dust temperature of $157 \pm 31 \mathrm{~K}$. The relative sparse coverage at far-IR and submillimetre wavelengths, coupled with the steep spectral slope between 450 and $850 \mu \mathrm{m}$, means that both $\lambda_{0}$ and $\beta$ are poorly constrained by the modelling. A fit to the long wavelength photometric points gives a characteristic dust temperature of $52 \mathrm{~K}$, and a lower limit on $\beta$ of 1.1. The estimated dust mass from the $850 \mu \mathrm{m}$ flux is $0.057 \mathrm{M}_{\oplus}$. Marshall et al. (2016) also suggest that there could actually be three distinct components to the disc, a warm inner element that fits the near-mid IR photometry (a radius of $\sim 20 \mathrm{au}$ ) and two annuli for the far-IR and submillimetre points (with radii of $\sim 80$ au and $\sim 270 \mathrm{au}$ ).

\subsection{Hd 84870}

HD 84870 is a luminous A3 star at a distance of $88 \mathrm{pc}$ with an estimated age of $100 \mathrm{Myr}$ (Rhee et al. 2007). At $850 \mu \mathrm{m}$, the emission peaks on the stellar position with a flux density of $6.2 \pm$ $1.0 \mathrm{mJy}$ (Fig. A7a). There are a couple of lower $\mathrm{S} / \mathrm{N}$ peaks also in the vicinity that are most likely background sources. Interpreting the emission as a disc about the star gives an upper limit to the disc radius from the $850 \mu \mathrm{m}$ image of $660 \mathrm{au}$.

The IR excess was originally detected by IRAS (Rhee et al. 2007). The disc has recently been resolved by Herschel/PACS revealing a ring-like structure in the star-subtracted $100 \mu \mathrm{m}$ image (Vican et al. 2016). Modelling of the disc indicates that the dust extends to a radius of 252 au from the star at a PA of $147^{\circ}$. The disc was not detected at $450 \mu \mathrm{m}$ but a fit to the other SED points gives a dust temperature of $50 \mathrm{~K}$, and constrains $\beta$ in the range of $0.3-1.2$. The estimated dust mass is $0.24 \mathrm{M}_{\oplus}$. There is also evidence for a warmer disc component at $146 \mathrm{~K}$, based on a fit to the near-mid IR data from AKARI (Ishihara et al. 2010), Spitzer (Chen et al. 2014) and WISE (Wright et al. 2010). 


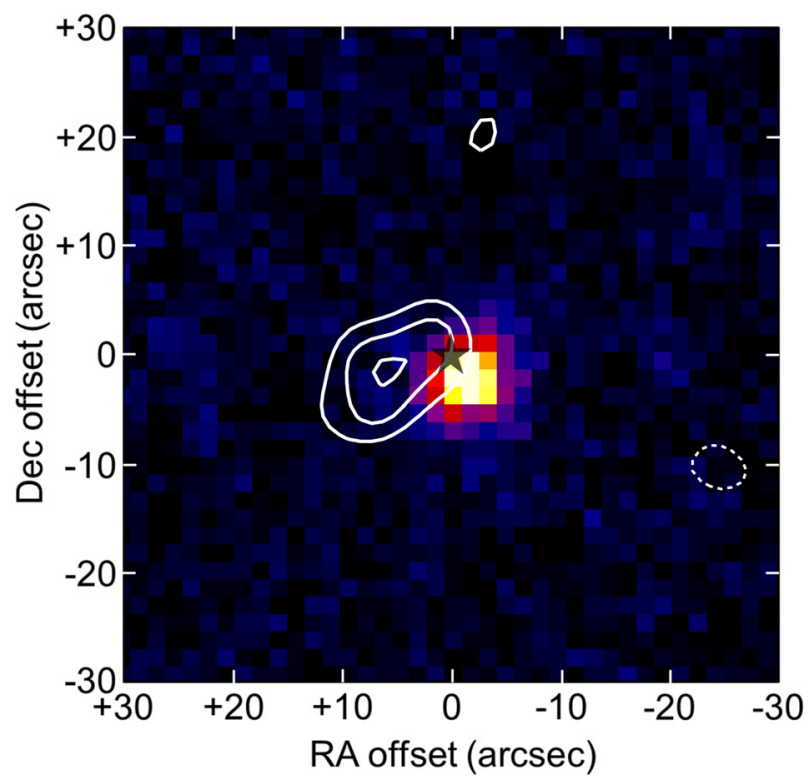

Figure 15. Herschel/PACS $70 \mu \mathrm{m}$ image of CE Ant (TWA 7) with the SONS survey $850 \mu \mathrm{m}$ contours overlaid. The contours start at $3 \sigma$ and increase in $1 \sigma$ steps. The Herschel image is taken from the Herschel Science Archive.

\subsection{CE Antliae (TWA 7, 2MASS J10423011-3340162)}

CE Ant (TWA 7) is an M2 dwarf star in the TW Hydrae Association, with an estimated age of $9 \mathrm{Myr}$ in a range spanning 3-20 Myr (Barrado y Navascués 2006). The distance of the star is uncertain but is likely to be similar to other members of the association $(\sim 50 \mathrm{pc})$. The $850 \mu \mathrm{m}$ image shows a clear, unresolved dust emission peak that is offset by approximately 6 arcsec to the east of the star (Fig. A7b). The offset is greater than the 3.3 arcsec expected due to statistical and positional uncertainties (see Section 6.4). The peak has a flux density of $7.2 \pm 1.3 \mathrm{mJy}$, consistent with the measurement made by SCUBA at $850 \mu \mathrm{m}$, of $9.7 \pm 1.6 \mathrm{mJy}$ (Matthews, Kalas \& Wyatt 2007b). The SCUBA measurement was carried out using photometry mode (Holland et al. 1999), and hence it is not possible to ascertain whether there was also an offset associated with that particular observation. Interpreting the structure as a disc about the star gives an upper limit to the radius from the $850 \mu \mathrm{m}$ image of $380 \mathrm{au}$.

$H S T /$ NICMOS imaging of the scattered light reveals an inclined disc extending to a radius of $1 \operatorname{arcsec}(\sim 35 \mathrm{au})$ at a PA of $53^{\circ}$ (Choquet et al. 2016). Modelling suggests that the observed structure is more likely to be a ring rather than a continuous disc. Herschel/PACS observations at $70 \mu \mathrm{m}$ resolve the disc with a deconvolved radius of $6.2 \operatorname{arcsec}(\sim 210 \mathrm{au})$ (Cieza et al. 2013). The Herschel/PACS image shows that the disc is reasonably well-aligned to the the stellar position, unlike the case for the SONS $850 \mu \mathrm{m}$ result (see Fig. 15). It is therefore possible that the peak seen at $850 \mu \mathrm{m}$ could be a background object, rather than a debris disc associated with CE Ant, although there is no evidence from Herschel observations that such an object exists at this position.

The SED contains both Spitzer/MIPS (Low et al. 2005) and Herschel photometry (Cieza et al. 2013) and is best fitted with two modified blackbodies, consistent with a previous analysis (RiviereMarichalar et al. 2013). The first blackbody fits the photometric points shortward of $\sim 100 \mu \mathrm{m}$ with a dust temperature of $81 \mathrm{~K}$ and suggests an inner disc at a radius of $2.5 \mathrm{au}$. Assuming that the observed structure at $850 \mu \mathrm{m}$ is a real disc associated with CE Ant,

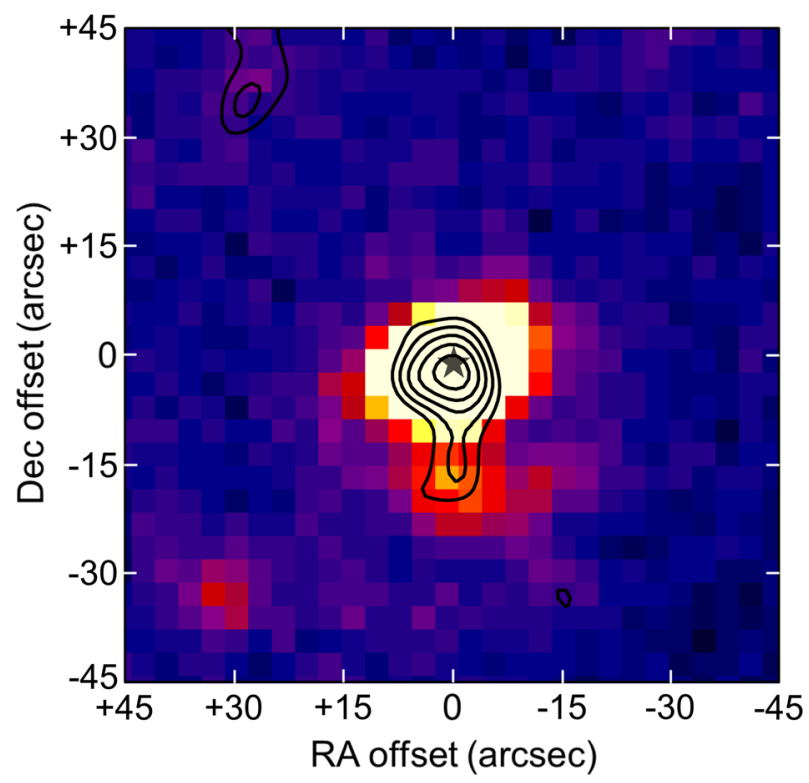

Figure 16. Herschel/PACS $160 \mu \mathrm{m}$ image of HD 92945 with the SONS survey $850 \mu \mathrm{m}$ contours overlaid. The contours start at $3 \sigma$ and increase in $1 \sigma$ steps. The Herschel image is taken from the Herschel Science Archive.

a second blackbody fit to the long-wavelength submillimetre photometry indicates the presence of a very cold structure, with a dust temperature of $19 \mathrm{~K}$, orbiting at a mean radius of $49 \mathrm{au}$, assuming that the dust grains have pure blackbody emission (both of which have large uncertainties, as indicated in Table 3). The value of $\beta$ is unconstrained due to the sparse photometric coverage, and lies in the range of $0-2.5$, whilst the estimated dust mass is $0.24 \mathrm{M}_{\oplus}$.

\subsection{HD 92945 (V419 Hya)}

HD 92945 is a K1 dwarf star at a distance of 21 pc with an estimated age of $200 \mathrm{Myr}$, lying between the lower and upper estimates of 100 and 300 Myr (Song, Zuckerman \& Bessell 2004; Chen et al. 2014), respectively. The $850 \mu \mathrm{m}$ SONS survey image shows a structure where the emission peaks at the stellar position, but with an apparent asymmetric extension to the south (Fig. A7c). The peak flux is $8.6 \pm 1.1 \mathrm{mJy}^{\text {beam }}{ }^{-1}$, that being consistent with the previously published result (Panić et al. 2013). The integrated flux, measured over an aperture of 40 arcsec diameter, is $12.6 \pm 1.5 \mathrm{mJy}$, confirming that the structure is resolved, if the extension to the south is really associated with a disc about the star. From the $850 \mu \mathrm{m}$ image, the measured deconvolved disc radius from the 2D Gaussian fitting is $11 \operatorname{arcsec}(\sim 210 \mathrm{au})$ in the major axis at a PA of $178^{\circ}$, i.e. dominated by the north-south extension.

The disc has been imaged in scattered light using HST/ACS, revealing an inclined, axisymmetric structure with an inner ring of radius $2-3^{\prime \prime}(\sim 43-65$ au) from the star and an outer disc declining in brightness to 5 arcsec $(\sim 110 \mathrm{au})$ roughly oriented east-west (Golimowski et al. 2011). Herschel/PACS imaging at 70 and $160 \mu \mathrm{m}$ reveals an extension (or possible second source) to the south, coincident with that seen in the $850 \mu \mathrm{m}$ image (Fig. 16). Given the asymmetric nature of the extension, it is more likely that this feature is caused by a background object, and is not part of a disc (see Section 6.5). In this scenario, the peak flux of $8.6 \pm 1.1 \mathrm{mJy} \mathrm{beam}^{-1}$, and an upper limit to the disc radius of $160 \mathrm{au}$, are better estimates to be adopted for a disc about the star. The SED contains both Spitzer/MIPS (Golimowski et al. 2011) and Herschel/PACS 
photometry (archival data), and using the $850 \mu \mathrm{m}$ peak flux, is well-fitted by a single temperature modified blackbody with a dust temperature of $42 \mathrm{~K}$, a $\beta$ in the range $0.4-1.1$, and a dust mass of $0.024 \mathrm{M}_{\oplus}$.

\subsection{Hd 98800}

HD 98800 is a pre-main-sequence K5 dwarf star in the TW Hydrae association, at a distance of $50 \mathrm{pc}$ with an estimated age of 9 Myr (Barrado y Navascués 2006). HD 98800 is actually a quadruple star system, containing a pair of spectroscopic binary stars (HD 98800A and B, separated by 36 au) and in which only HD 98800B harbours a circumstellar disc (Torres et al. 1995; Koerner et al. 2000). Together with Fomalhaut Section 3.3, the $850 \mu$ m image represents by far the brightest disc in the SONS survey with a flux density of $94 \pm 1.5 \mathrm{mJy}$ (Fig. A7d). The disc is also well-detected at $450 \mu \mathrm{m}$, revealing an unresolved disc with a flux density of $242 \pm 14 \mathrm{mJy}$. The upper limit to the disc radius from the $450 \mu \mathrm{m}$ image is 255 au.

The HD 98800B disc has been resolved by the SMA revealing that it is closely aligned with the orbit of the spectroscopic binary (HD 98800A), and extends to a radius of only 10-15 au from HD $98800 \mathrm{~B}$ at a PA of $158^{\circ}$ (Andrews et al. 2010). The disc has a fractional luminosity of $\sim 0.1$ and so is outside the generally accepted range for a debris disc (see Section 4.4). Indeed, it is commonly referred to as a 'transitional disc', i.e. a disc evolving between the younger protoplanetary and the older debris phases. The SED in the IR is well-characterized by WISE (Wright et al. 2010), AKARI (Ishihara et al. 2010) and Spitzer/MIPS photometry (Chen et al. 2014). In the submillimetre, the photometry has considerable more scatter, with seemingly low flux estimates at $870 \mu \mathrm{m}$ from APEX/LABOCA (a $4 \sigma$ detection; Nilsson et al. 2010) and $1.3 \mathrm{~mm}$ from JCMT/UKT14 (also a $4 \sigma$ detection; Sylvester et al. 1996), compared to the SED fit. The near blackbody fit suggests a dust temperature of $156 \mathrm{~K}$, a well-constrained value of $\beta$ in the range 0.0 to 0.1 , and an estimated dust mass of $0.37 \mathrm{M}_{\oplus}$.

\subsection{Hd 104860}

HD 104860 is a F8 star located at a distance of 46 pc with an estimated age of $200 \mathrm{Myr}$ (Morales et al. 2013). The $850 \mu \mathrm{m}$ image (Fig. A8a) shows a structure in which the peak emission is well-centred on the stellar position. The peak flux density is $6.5 \pm$ $1.0 \mathrm{mJy}$ beam $^{-1}$, in good agreement with previous SCUBA flux estimates (Najita \& Williams 2005). The integrated flux, measured in a 50 arcsec diameter aperture centred on the star, is $8.7 \pm 1.4 \mathrm{mJy}$, indicating the overall structure could be marginally resolved. Another possibility, however, given the asymmetry of the structure, is that the $4 \sigma$ peak to the east may be a background object. Interpreting the emission as that from a disc gives an upper limit to the radius from the $850 \mu \mathrm{m}$ image of 340 au (assuming an unresolved structure).

The disc has been resolved by Herschel/PACS, with the modelled image at $160 \mu \mathrm{m}$ suggesting a slightly elongated disc of radius $3.5 \operatorname{arcsec}(\sim 166 \mathrm{au})$ from the star at a PA of $12^{\circ}$ (Morales et al. 2013). There is also a hint of a slight eastward extension to the image at $160 \mu \mathrm{m}$, which is coincident with the second peak seen in the $850 \mu \mathrm{m}$ image, perhaps supporting the hypothesis that this is a background object. The disc has also been resolved by the SMA at $1.3 \mathrm{~mm}$, revealing a disc with a peak in emission at a radius of $2.5 \operatorname{arcsec}(\sim 110 \mathrm{au})$ and elongated at a PA roughly in a northsouth direction (Steele et al. 2016). The SMA images, however, show no signs of a second source to the east. The SED in the IR is well characterized by Spitzer/MIPS (Hillenbrand et al. 2008), in the far-IR by Herschel/PACS (Morales et al. 2013), CSO and IRAM (Roccatagliata et al. 2009) in the submillimetre/near-millimetre, and the VLA at $9 \mathrm{~mm}$ (MacGregor et al. 2016a). In fitting the SED it has been assumed that the flux at $850 \mu \mathrm{m}$ is best represented by the peak value, giving a dust temperature of $47 \mathrm{~K}$, a $\beta$ index in the range of $0.4-1.2$, and an estimated dust mass of $0.071 \mathrm{M}_{\oplus}$.

\subsection{Hd 107146}

HD 107146 is a young solar analogue star (G2V) located at a distance of $28.5 \mathrm{pc}$ with an estimated age of $100 \mathrm{Myr}$ (Chen et al. 2014), although it could be as young as $30 \mathrm{Myr}$ (Williams et al. 2004). The $850 \mu \mathrm{m}$ image (Fig. A8b) reveals a bright structure, with a peak flux of $20.6 \pm 2.1 \mathrm{mJy}^{\text {beam }}{ }^{-1}$ slightly offset by $\sim 4$ arcsec from the star. The structure appears slightly extended to the south-west, although the integrated flux, measured over a 40 arcsec diameter aperture, indicates that this feature is not significant compared to the peak flux value. Interpreted as a disc, the upper limit to the radius from the $850 \mu \mathrm{m}$ image is $210 \mathrm{au}$. The measurement is in excellent agreement with the previous SCUBA measurement of $20 \pm 4 \mathrm{mJy}$, and the disc was also resolved by SCUBA at $450 \mu \mathrm{m}$ with a deconvolved radius of 150 au from the star at a PA of $155^{\circ}$ (Williams et al. 2004).

The disc was first detected in scattered light using HST/ACS, showing a circularly symmetric structure with maximum opacity occurring at $130 \mathrm{au}$, with a PA of $\sim 160^{\circ}$ and inclined at $25^{\circ}$ to the plane of the sky (Ardila et al. 2004). The scattered light images also reveal an object $\sim 7$ arcsec south-west of the star, which was identified as a spiral galaxy. In the $9 \mathrm{yr}$ between the HST and SCUBA-2 observations, the proper motion of the star means that the separation to the galaxy is now 5 arcsec. Hence, it is possible that the SONS survey image at $850 \mu \mathrm{m}$ is contaminated by the presence of a background galaxy causing the apparent extension to the disc.

SMA observations at $880 \mu \mathrm{m}$ show that the emission is distributed in a ring of radius 3.5 arcsec from the star, and this emission is modelled with inner and outer radii of 50 and 170 au with a disc PA of $148^{\circ}$ (Hughes et al. 2011). The total flux measured by the SMA was $36 \pm 1 \mathrm{mJy}$, significantly higher than the SONS result at $850 \mu \mathrm{m}$. Given the 5 arcsec separation between the star and the galaxy, it is possible that the total SMA emission also contains flux from the background source. Similarly, with ALMA at $1.3 \mathrm{~mm}$ the disc is seen extending from 30 to 150 au at a PA of $144^{\circ}$, whilst models suggest a clear decrease in the dust emission at a radius of $\sim 80$ au extending over a width of 9 au (Ricci et al. 2015a). The total flux density for the disc of $12.5 \pm 1.3 \mathrm{mJy}$ from the ALMA observations may also be contaminated by flux from the nearby galaxy. Given that the star is a young Solar analogue, the disc could be a larger version of the Edgeworth-Kuiper belt in our Solar system (Ricci et al. 2015a).

The emission from the disc is well-sampled in the IR through millimetre region, including measurements from Spitzer/MIPS (Chen et al. 2014), Herschel/PACS (Morales et al. 2016), CSO (Corder et al. 2009), OVRO (Carpenter et al. 2005) and ACTA (Ricci et al. $2015 \mathrm{~b}$ ). The fit to the SED, using the peak flux at $850 \mu \mathrm{m}$, tightly constrains $\beta$ to a value of between 0.8 and 1.0 and gives a dust temperature of $41 \mathrm{~K}$, leading to an estimated dust mass of $0.093 \mathrm{M}_{\oplus}$. The fit to the SED at near-mid IR wavelengths also suggests an inner, 'warm' component radiating at $81 \mathrm{~K}$. 


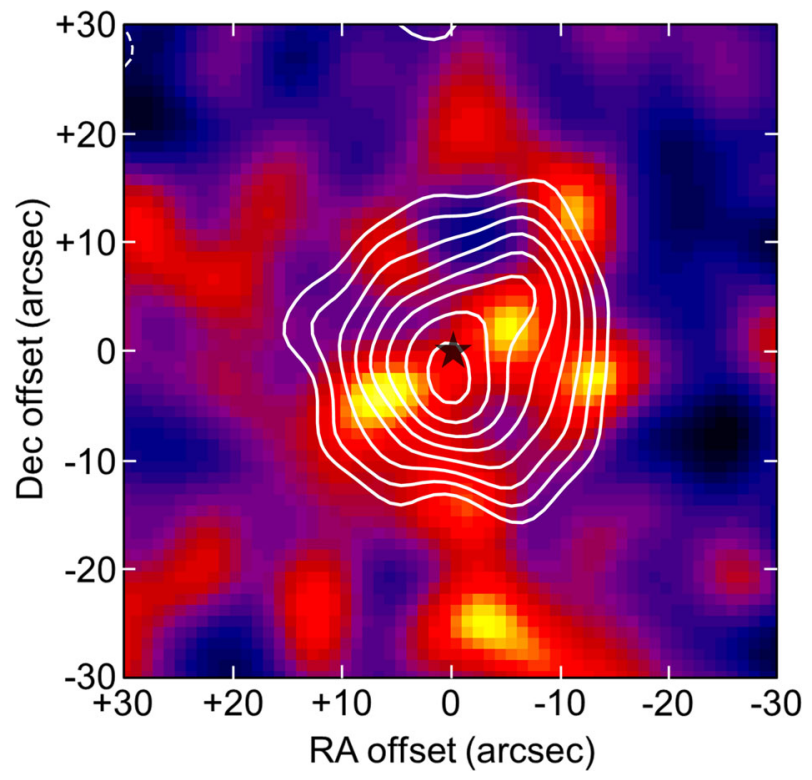

Figure 17. The $450 \mu \mathrm{m}$ image from observations of HD 109085 ( $\eta$ Crv) with contours from the $850 \mu \mathrm{m}$ image overlaid. The contours and symbols are as described in Fig. 7.

\section{$5.31 \eta$ Corvi (HD 109085)}

$\eta$ Crv (HD 109085) is a nearby F2 star located at a distance of $18 \mathrm{pc}$ with an estimated age of $1380 \mathrm{Myr}$ (Chen et al. 2014) in a range of 1000-2000 Myr (Vican 2012). The images and fluxes presented in this paper are slightly updated versions of those first published by Duchêne et al. (2014) following a re-analysis of the data. The $850 \mu \mathrm{m}$ image (Fig. A8c) shows emission peaking with a flux of $7.2 \pm 0.7 \mathrm{mJy}$ beam $^{-1}$. The integrated flux density of $15.4 \pm 1.1 \mathrm{mJy}$, measured in a 40 arcsec diameter aperture centred on the star, in good agreement with previous SCUBA flux estimates (Wyatt et al. 2005). Interpreting the emission as a disc about the star gives deconvolved major and minor axis radii of 10.4 and $7.5 \operatorname{arcsec}(190$ and $135 \mathrm{au})$, respectively, at a PA of $132^{\circ}$ and inclined to the plane of the sky by $44^{\circ}$. The SONS survey image at $450 \mu \mathrm{m}$ is low $\mathrm{S} / \mathrm{N}$ due to the relatively poor weather conditions at the time of the observations, but shows that the disc is resolved into the two prominent clumps seen previously (Wyatt et al. 2005) albeit only at the $\sim 4 \sigma$ significance level (Fig. 17).

The first IR excess flux measurements of $\eta \mathrm{Crv}$ were made by IRAS (Stencel \& Backman 1991), and the disc has been observed at many wavelengths over the past two decades. SCUBA observations at $450 \mu \mathrm{m}$ resolved the disc for the first time revealing a ringlike structure oriented at PA of $130^{\circ}$ with model fits indicating a disc inclination of $45^{\circ}$ and a radius of 150 au (Wyatt et al. 2005). Herschel/PACS images at $70 \mu \mathrm{m}$ show a central flux peak on the star surrounded by an inclined ring at $\sim 47^{\circ}$ to the plane of the sky (Duchêne et al. 2014), the latter being the same emission seen in the submillimetre. Modelling of the disc suggests that there are both warm and cold dust belts in the system, the cold component peaking in emission at 164 au with a estimated width of $\sim 9$ au, at a PA of $116^{\circ}$ (Duchêne et al. 2014).

More recently, ALMA observations at $880 \mu \mathrm{m}$ reveal an asymmetric belt of emission of mean radius of $8.4 \operatorname{arcsec}(\sim 152 \mathrm{au})$ with a width of $2.6 \operatorname{arcsec}(\sim 46 \mathrm{au})$, at a PA of $117^{\circ}$ and an inclination of $35^{\circ}$ (Marino et al. 2017a). The total flux emission measured from the ALMA image of $10.1 \pm 0.4 \mathrm{mJy}$ is $2 \sigma-3 \sigma$ lower than would

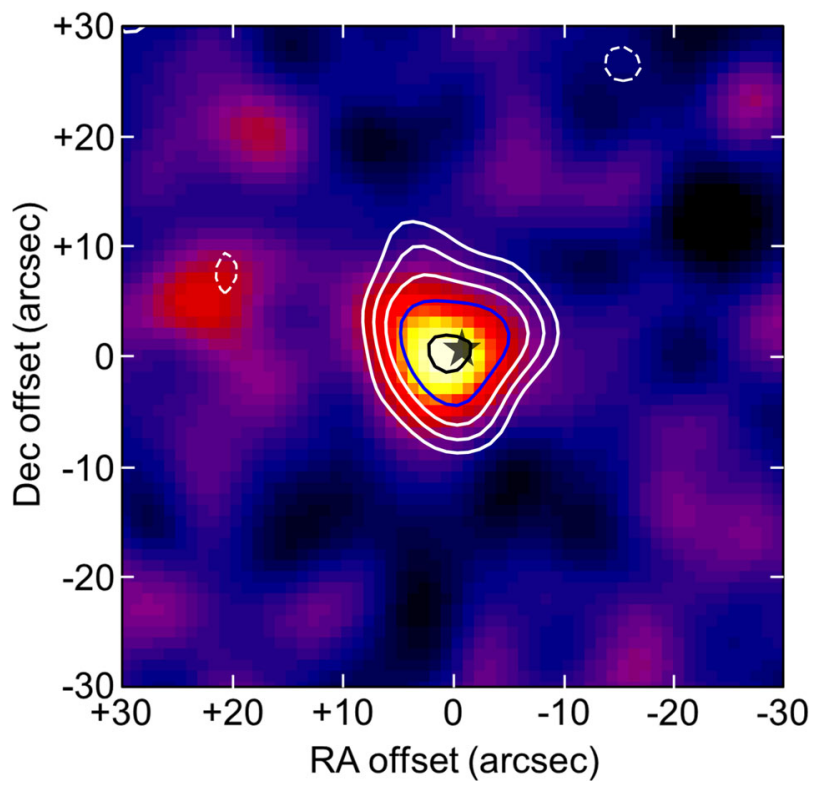

Figure 18. The $450 \mu \mathrm{m}$ image from observations of HR 4796 (TWA 11; HD 109573) with contours from the $850 \mu \mathrm{m}$ image overlaid. The contours and symbols are as described in Fig. 7.

be expected compared to the SCUBA-2 $850 \mu \mathrm{m}$ flux if extrapolated to $880 \mu \mathrm{m}$, assuming a spectral index of 3 . As pointed out by Marino et al. (2017a), the difference could be due either to extended emission being missed by ALMA as a result of having an insufficient number of short baselines, or due to the image reconstruction method adopted for the ALMA data.

The photometric data from the SED is fitted by a two-component model. The fit to the near-mid IR data suggests an inner belt at a radius of $\sim 3$ au composed of warm dust radiating at $254 \mathrm{~K}$, whilst the fit to far-IR/submillimetre data indicates the presence of a cold belt with a characteristic dust temperature of $45 \mathrm{~K}$, and a $\beta$ in the range of $0-0.7$, leading to an estimated dust mass of $0.028 \mathrm{M}_{\oplus}$.

\subsection{HR 4796 (TWA11, HD 109573)}

HR 4796 (HD 109573) is a binary star system in the constellation of Centaurus, at a distance of $73 \mathrm{pc}$ and is part of the TW Hydrae Moving Group with an age of 9 Myr (Barrado y Navascués 2006). The two stars of the system are separated by 7.7 arcsec, with the primary having a spectral type A0, whilst the smaller companion is a red dwarf of type M2.5. Emission is detected at both 850 and $450 \mu \mathrm{m}$ with flux densities of $14.4 \pm 1.9$ and $117 \pm 21 \mathrm{mJy}$, respectively (Fig. A8d and Fig. 18). Interpreting the emission as a disc structure about the star gives an upper limit to the radius from the $450 \mu \mathrm{m}$ image is $370 \mathrm{au}$.

The disc around HR 4796 was first resolved in the mid-IR (Jayawardhana et al. 1998; Koerner et al. 1998) at 12.5 and $20 \mu \mathrm{m}$ revealing an elongated structure with the dust peaking at a radius of 70 au surrounding the central primary star. The PA of the disc was measured at $30^{\circ}$, consistent with the binary companion, and suggesting that the disc-binary system is being seen nearly along the orbital plane. The disc was interpreted as a ring-like structure, having two lobes similar to HD 216956 (Fomalhaut), with an inner hole devoid of dust extending to a radius of 55 au. The disc has also been imaged in scattered light using HST/NICMOS and STIS, which show a distinctive ring-like symmetrical structure, consistent with the mid-IR observations (Schneider et al. 1999, 2009). The 
images revealed that the ring has a peak intensity at $70 \mathrm{au}$, with a resolved width of $17 \mathrm{au}$, and a major axis lying at a PA of $27^{\circ}$. The observed brightness variation across the disc in thermal emission has been attributed to pericentre glow, in which the asymmetry is caused by the gravitational perturbations from an unseen planetary system (Wyatt et al. 2000).

The SED is well-sampled from the near-IR to the millimetre and includes photometric points from both Spitzer (Chen et al. 2014) and WISE (Wright et al. 2010). Fitting the SED suggests a very well-defined, single temperature disc of $99 \mathrm{~K}$, and a $\beta$ value of $0.7-1.0$, and an estimated dust mass of $0.19 \mathrm{M}_{\oplus}$ from the $850 \mu \mathrm{m}$ flux.

\subsection{Virginis (HD 115617)}

HD 115617 (61 Vir) is a nearby main sequence star (G7-V) hosting a system of at least two known planets, both of which are subSaturn mass and have orbits of less than $0.5 \mathrm{AU}$. The star lies at a distance $8.6 \mathrm{pc}$ with an estimated age of $6300 \mathrm{Myr}$, within a range of 6100-6600 Myr (Mamajek \& Bell 2014). The SONS survey image at $850 \mu \mathrm{m}$ reveals a main emission peak a few arcseconds offset from the star together with a second isolated $3.5 \sigma$ peak 12 arcsec to the north (Fig. A9a). The peak flux for the main structure is $3.9 \pm$ $0.8 \mathrm{mJy}$ and is possibly marginally resolved with an integrated flux of $5.8 \pm 1.0 \mathrm{mJy}$ measured in a 40 arcsec diameter aperture centred on the star, but not including the peak to the north. The image reported here benefits from further integration time than the image published by Panić et al. (2013). The structure has a PA of $\sim 66^{\circ}$ and interpreting as a disc about the star gives a deconvolved radius of $4.7 \operatorname{arcsec}(\sim 40 \mathrm{au})$. The updated SONS results are reported in Marino et al. (2017b).

61 Vir was also included in a survey of FGK stars by Spitzer that detected the disc at 24 and $70 \mu \mathrm{m}$ (Tanner et al. 2009). Herschel has provided the most definitive study of the HD 115617 debris disc so far with images from 70 to $500 \mu \mathrm{m}$ as part of the DEBRIS survey. The images show a nearly edge-on configuration extending out to at least 100 au from the star at a PA of $\sim 65^{\circ}$, with the dust population peaking in density in a belt between 30 and 100 au (Wyatt et al. 2012). Thus, there is expected to be very little interaction between the disc and the known planets, although theory suggests that planetesimal belts such as this one might hold the key to understanding super-Earth systems such as HD 115617. The northern source, seen in the SONS $850 \mu \mathrm{m}$ image, is most likely a background object based on the interpretation of the Herschel/PACS $160 \mu \mathrm{m}$ image (Wyatt et al. 2012). Observations with ALMA at $870 \mu \mathrm{m}$ resolve three compact sources (Marino et al. 2017b), one of which seems coincident with the northern source seen in both SONS $850 \mu \mathrm{m}$ and Herschel/PACS $160 \mu \mathrm{m}$ images. By combining SCUBA-2 and ALMA observations, it is inferred that the disc is likely very extended covering radius range of 30 au to at least $150 \mathrm{au}$, implying the existence of a broad parent planetesimal belt.

The SCUBA-2 $850 \mu \mathrm{m}$ result seems to indicate a rapid fall off in flux towards the millimetre region $\left(\lambda_{0}>650 \mu \mathrm{m}\right)$, although this sharp decrease is not totally consistent with the Herschel/SPIRE photometry. Hence the fit to the SED is not convincing with a characteristic dust temperature of $65 \mathrm{~K}$, and only constrains $\beta$ in the range between 0 and 1.5. The dust mass is estimated to be $1.6 \times$ $10^{-3} \mathrm{M}_{\oplus}$. The radius of the disc, derived from the SED fit and assuming blackbody grain properties, is only 17 au, significantly smaller than the value of 40 au obtained from the marginally resolved $850 \mu \mathrm{m}$ image.

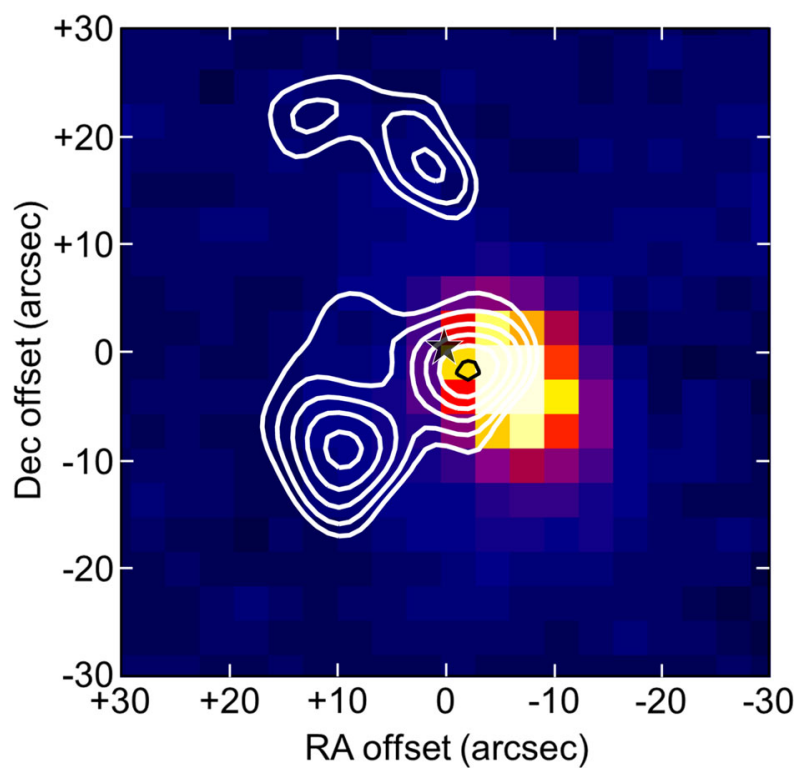

Figure 19. Herschel/PACS $160 \mu \mathrm{m}$ image of HD 127821 with the SONS survey $850 \mu \mathrm{m}$ contours overlaid. The Herschel image is taken from the Herschel Science Archive.

\section{$5.34 \lambda$ Boötis (HD 125162)}

HD 125162 ( $\lambda$ Boo) is a luminous A0 star at a distance $30 \mathrm{pc}$ with an estimated age of $2800 \mathrm{Myr}$, but with a large uncertainly stretching from 2000 to $3900 \mathrm{Myr}$ (Montesinos et al. 2009). At $850 \mu \mathrm{m}$, unresolved emission is detected, well-centred on the stellar position, with a flux density of $3.9 \pm 0.8 \mathrm{mJy}$ (Fig. A9b). If interpreted as a disc about the star, the upper limit to the radius from the $850 \mu \mathrm{m}$ image is $230 \mathrm{au}$.

The HD 125612 debris disc has been resolved by Herschel/PACS at $70 \mu \mathrm{m}$ revealing a slightly elongated structure of radius 40 au at a PA of $42^{\circ}$ (Booth et al. 2013). Photometry from Spitzer (Chen et al. 2014) also helps to define the mid-IR section of the SED. The $850 \mu \mathrm{m}$ photometric point helps to anchor the SED fit to give a characteristic dust temperature of $87 \mathrm{~K}$, but still a poorly constrained $\beta$ value in the range of $0.3-1.8$. The dust mass is estimated to be $0.010 \mathrm{M}_{\oplus}$.

\subsection{Hd 127821}

HD 127821 is an F4 star at a distance of $32 \mathrm{pc}$ with an estimated age of $1020 \mathrm{Myr}$ (Chen et al. 2014), although it could be as old as 3400 Myr (Moór et al. 2006). The SONS image at $850 \mu \mathrm{m}$ appears to show a predominantly double-peaked structure with one peak reasonably well centred on the stellar position, and the other offset by $\sim 13$ arcsec to the south-east (Fig. A9c). The peak nearest to the star has a flux of $5.8 \pm 0.7 \mathrm{mJy}_{\text {beam }}^{-1}$. The integrated flux in a 40 arcsec diameter aperture, centred between the two peaks, is $10.5 \pm 1.4 \mathrm{mJy}$. This flux is just consistent with previous SCUBA photometry of $13.2 \pm 3.7 \mathrm{mJy}$ beam $^{-1}$ (Williams \& Andrews 2006), noting also the slightly larger FWHM beam for SCUBA. In addition, given that the two peaks are approximately the same brightness, and that the integrated flux is equal to their combined levels, it is most likely that these are two separate sources. There is also other structure seen to the north, offset from the star by $\sim 18$ arcsec.

Herschel imaging with PACS and SPIRE at 160 and $250 \mu \mathrm{m}$ reveals a single source, well-aligned with the central peak in the SONS $850 \mu \mathrm{m}$ image. Fig. 19 shows the Herschel/PACS $160 \mu \mathrm{m}$ 


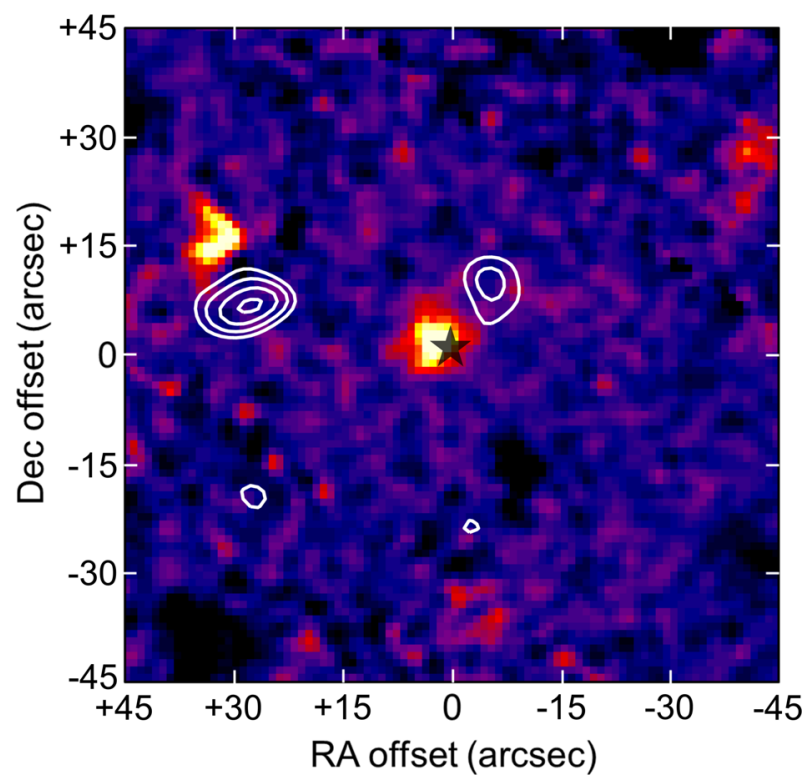

Figure 20. Herschel/PACS $160 \mu \mathrm{m}$ image of HD 128167 ( $\sigma$ Boo) with the SONS survey $850 \mu \mathrm{m}$ contours overlaid. The Herschel image is taken from the Herschel Science Archive.

image with the SONS survey $850 \mu \mathrm{m}$ contours overlaid. Based on these observations, it is therefore possible that the peak to the south-east (as well as the one to the north) is indeed background objects, although these are not seen in the Herschel images. It is therefore likely that only the central peak is representative of a disc, and under this assumption (i.e. only using the flux of the central peak at $850 \mu \mathrm{m}$ ) the SED can be fitted with a single-temperature $47 \mathrm{~K}$ modified blackbody and a $\beta$ value in the range $0.9-1.9$. The estimated dust mass is $0.031 \mathrm{M}_{\oplus}$, whilst the upper limit to the disc radius from the $850 \mu \mathrm{m}$ image is $235 \mathrm{au}$.

\section{$5.36 \sigma$ Boötis (HD 128167)}

HD 128167 ( $\sigma$ Boo) is an F2 star at a distance of 16 pc with an estimated age of $1000 \mathrm{Myr}$ but it could be as old as $4780 \mathrm{Myr}$ (Rhee et al. 2007). The SONS survey image at $850 \mu \mathrm{m}$ shows emission, offset from the star by $\sim 11$ arcsec to the north-west, with a flux density of $4.1 \pm 0.9 \mathrm{mJy}$ (Fig. A9d). Given the large offset, it is unlikely that this peak is associated with the star and is probably a separate source. Observations of HD 128167 with SCUBA at $850 \mu \mathrm{m}$ reported a flux of $6.2 \pm 1.7 \mathrm{mJy}$ (Sheret et al. 2004). These observations used the technique of 'extended photometry', in which a 9-point map was made around the source with a spacing of 5 arcsec. Such a map would have detected flux from the offset position shown in the SCUBA-2 image. Unpublished imaging observations with SCUBA also seem to indicate an offset peak in the same direction (Wyatt, private communication).

Herschel/PACS observations at $160 \mu \mathrm{m}$ reveal a peak at $17.5 \pm 3.5 \mathrm{mJy}$ from a position coincident with the star (Fig. 20). A second peak detected in the PACS image to the north-east is $\sim 8.5$ arcsec offset from the brighter peak in the $850 \mu \mathrm{m}$ SONS image. If a rotational shift of $\sim 25^{\circ}$ was applied in either image, then there would be close alignment between the peak near the centre of the field and that to the east. Given the accuracy of pointing and image reconstruction for both telescope facilities, such problems would be unlikely explanations for the discrepancies reported here. It is therefore concluded that the $\sigma$ Boo disc was not detected by

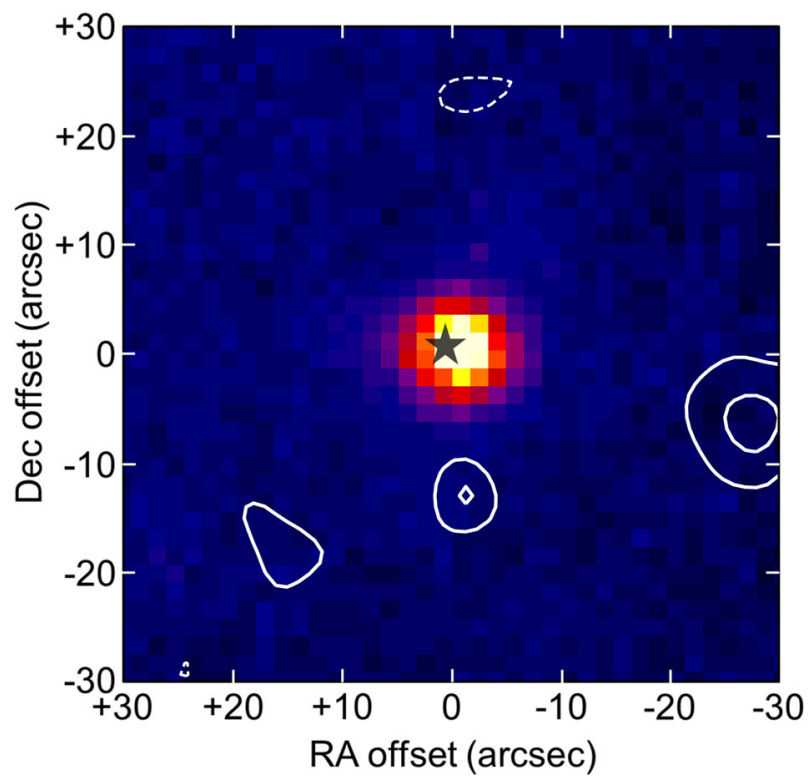

Figure 21. Herschel/PACS $100 \mu \mathrm{m}$ image of HD 141378 with the SONS survey $850 \mu \mathrm{m}$ contours overlaid. The contours start at $3 \sigma$ and increase in $1 \sigma$ steps. The Herschel image is taken from the Herschel Science Archive.

SCUBA-2 and the peak seen to the north-west is a likely background object. The SED (Fig. A9a) only includes photometric points from the position at the star (i.e. the SCUBA-2 $850 \mu \mathrm{m}$ point is presented as an upper flux limit). If the fit is truly representative of the SED at long wavelengths, then the disc would have a flux density of significantly less than $1 \mathrm{mJy}$ at $850 \mu \mathrm{m}$ (i.e. below the sensitivity threshold of this observation).

\subsection{Hd 141378}

HD 141378 is a luminous A5 star at a distance of 54 pc with an estimated age of $150 \mathrm{Myr}$ (Rieke et al. 2005). The SONS survey $850 \mu \mathrm{m}$ image reveals a number of features, all of which are offset from the star to the south (Fig. A10a). The nearest peak is 13 arcsec offset and has a flux density $8.5 \pm 1.8 \mathrm{mJy}$. This offset is far in excess of what would be expected for a disc about the star, allowing for positional and statistical uncertainties in the measurement (see Section 6.4). It is therefore likely that this peak is due to a separate source, perhaps a background galaxy.

Herschel/PACS imaging at 100 and $160 \mu \mathrm{m}$ reveals an emission peak that is well-centred on the star position, and does not show any evidence of the multiple peaks seen at $850 \mu \mathrm{m}$ to the south (see Fig. 21). Furthermore, the SED fit to the Herschel photometry suggests that the disc would not have been detected by SCUBA2 at the sensitivity levels reached in the observation. Hence, it is probable that a debris disc does exist around HD 141378, but would be very faint at $850 \mu \mathrm{m}(\sim 2 \mathrm{mJy})$.

\subsection{Serpentis (HD 143894)}

HD 143894 (44 Ser) is a luminous A3 star at a distance of 55 pc and has an estimated age of $300 \mathrm{Myr}$ (Rhee et al. 2007) although it could be as old as $530 \mathrm{Myr}$ (Chen et al. 2014). The SONS $850 \mu \mathrm{m}$ image clearly shows emission with a peak flux density of $5.6 \pm$ $0.9 \mathrm{mJy} \mathrm{beam}^{-1}$ (Fig. A10b), but offset from the stellar position by $\sim 4$ arcsec (slightly above the level of 3.1 arcsec expected due to positional and statistical uncertainties; Section 6.4). The integrated 
flux in a 40 arcsec diameter aperture, centred on the stellar position, is $10.1 \pm 1.2 \mathrm{mJy}$, indicating that, even at a distance of $55 \mathrm{pc}$, the emission is resolved. Fitting the radial extent of the emission gives a deconvolved radius of $10.2 \operatorname{arcsec}(\sim 560 \mathrm{au})$ at a PA of $70^{\circ}$, almost four times the radius of 150 au derived assuming the dust grains have pure blackbody emission. If the emission is indeed indicative of a disc, it represents a very large structure about the star (i.e. $10 \times$ the size of the Edgeworth-Kuiper belt in our Solar system). Given the offset between the star and flux peak, one possibility is that the morphology could be somewhat distorted by a background object, resulting in an artificially enlarged structure. Further observations are needed to resolve this issue.

This target was placed in the 'hard to quantify' flux category, having a very poorly constrained dust temperature due to lack of far-IR photometry. Hence, the SED is sparsely defined with only photometry from Spitzer/MIPS at $24 \mu \mathrm{m}$ (Chen et al. 2014) and IRAS at $60 \mu \mathrm{m}$ contributing to the disc model SED fit. The $850 \mu \mathrm{m}$ flux helps to constrain $\beta$ to a value in the range $0-1.1$. A temperature of $53 \mathrm{~K}$ and a dust mass of $0.14 \mathrm{M}_{\oplus}$ are derived from the fit and $850 \mu \mathrm{m}$ flux, respectively.

\subsection{Herculis (HD 150378)}

HD 150378 (37 Her) is a luminous A1 star at a distance of 90 pc with an estimated age of $200 \mathrm{Myr}$ (Chen et al. 2014). The SONS survey image at $850 \mu \mathrm{m}$ shows a bright peak, offset from the stellar position by 17 arcsec with a flux density of $10.2 \pm 1.1 \mathrm{mJy}$ (Fig. A10c). This emission is highly unlikely to be associated with the star and is probably another object, although no identification has been possible from standard catalogues. There is also an IRAS detection of excess far-IR radiation at $60 \mu \mathrm{m}$, but the large IRAS beamsize means it is impossible to ascertain whether this is the same object as detected by SCUBA-2 (the most likely scenario) or a true debris disc associated with the star. The SED contains Spitzer/IRS photometry that suggests the possibility of a warm, inner disc component. The fit to the two long wavelength photometric points in the SED (Fig. A10c) suggests an object with a dust temperature of $32 \mathrm{~K}$. Given the large offset in the $850 \mu \mathrm{m}$ image, it is concluded that there is no strong evidence for a cold debris disc surrounding HD 150378 based on the far-IR and submillimetre data.

\subsection{Herculis (HD 150682)}

HD 150682 (39 Her) is an F3 star at a distance of 44 pc with an estimated age of $1700 \mathrm{Myr}$ (Chen et al. 2014). The SONS survey image at $850 \mu \mathrm{m}$ shows an unresolved peak, well-centred on the star with a flux density of $5.5 \pm 0.9 \mathrm{mJy}$ (Fig. A10d). The upper limit to the disc radius from the $850 \mu \mathrm{m}$ image is $330 \mathrm{au}$. The only other far-IR photometry detection is from Spitzer/MIPS at $70 \mu \mathrm{m}$ (Trilling et al. 2007). The fit to the poorly sampled SED suggests a cold component disc with a dust temperature of $32 \mathrm{~K}$ (but with a large error of $\pm 14 \mathrm{~K}$ ), $\beta$ in the range from 0.0 to 2.2 , and an estimated dust mass of $0.081 \mathrm{M}_{\oplus}$.

\subsection{Hd 158352}

HD 158352 is a luminous A8 star at a distance of $60 \mathrm{pc}$ with an estimated age of $750 \mathrm{Myr}$, with an uncertainty of around $\pm 150 \mathrm{Myr}$ (Moór et al. 2006). The SONS image at $850 \mu \mathrm{m}$ shows an unresolved peak, well-centred on the star, with a flux density of $5.3 \pm 1.0 \mathrm{mJy}$ (Fig. A11a). Interpreting the emission as being representative of a disc about the star gives an upper limit to the disc radius from the
$850 \mu \mathrm{m}$ image of $450 \mathrm{au}$. There is another peak some $25 \operatorname{arcsec}$ to the north-west that is slightly brighter. The only other far-IR photometry detection is from Spitzer/MIPS at 24 and $70 \mu \mathrm{m}$ (Chen et al. 2014) and so $\lambda_{0}$ and $\beta$ are not well constrained. The fit to the SED suggests a single-component disc with a dust temperature of $62 \mathrm{~K}$, and an estimated dust mass of $0.075 \mathrm{M}_{\oplus}$.

\section{$5.42 \gamma$ Ophiuchi (HD 161868)}

HD 161868 ( $\gamma \mathrm{Oph})$ is a luminous A0 star at a distance of $32 \mathrm{pc}$ with an estimated age of $185 \mathrm{Myr}$ with lower and upper limits of 50 and 277 Myr, respectively (Song et al. 2001). There are, however, other studies that suggest the star could be as old as $450 \mathrm{Myr}$ (Moór et al. 2015). The $850 \mu \mathrm{m}$ observations reported in this paper are deeper than those previously published (Panić et al. 2013), and show a marginally extended structure, with the peak emission having a flux density of $4.8 \pm 0.8 \mathrm{mJy}^{\text {beam }}{ }^{-1}$ (Fig. A11b). The integrated flux, measured in an aperture of 50 arcsec in diameter and centred on the peak of the emission is $7.1 \pm 1.0 \mathrm{mJy}$, indicating that the structure is resolved. Interpreted as a disc about the star, the radial extent gives a deconvolved radius of $7.8 \operatorname{arcsec}(\sim 250 \mathrm{au})$ at a PA of $75^{\circ}$, compared to $\sim 85$ au assuming pure blackbody emission from the dust grains (see Table 3).

Spitzer/MIPS observations first resolved the disc showing dust extending out to a large radius of $18 \operatorname{arcsec}(\sim 520 \mathrm{au})$ at $70 \mu \mathrm{m}$, with an inclination angle to the plane of the sky of $\sim 50^{\circ}$ and a PA of $55^{\circ}$ (Su et al. 2008). These data suggest a disc almost twice as large as the SCUBA-2 observations indicate. Herschel/PACS observations showed a disc of radius 4.3-5.5 $\operatorname{arcsec}(\sim 135-172 \mathrm{au})$ over the observed wavelength range of 70-160 $\mu \mathrm{m}$ (Moór et al. 2015 ), inclined at $58^{\circ}$ to the plane of the sky and at a PA of $61^{\circ}$ (Moór et al. 2015). Subsequent modelling of the Herschel/PACS results suggested inner and outer radii for the disc of 49 and 278 au, respectively (Moór et al. 2015). A background object has also been identified at a position 21 arcsec to the east of the star (Moór et al. 2015), and hence it is possible that the low-level emission (which is less than $3 \sigma$ significance) seen in the $850 \mu \mathrm{m}$ image at this position is due to this.

The far-IR SED is well constrained by measurements from Spitzer/MIPS (Su et al. 2008; Chen et al. 2014) and Herschel observations from PACS and SPIRE (Pawellek et al. 2014; Moór et al. 2015). The fit to the SED suggests a dual-component disc with the cold component having a characteristic dust temperature of $68 \mathrm{~K}$, a $\beta$ value in the range of $0.8-1.4$, and an estimated dust mass of $0.025 \mathrm{M}_{\oplus}$. The warmer component, fitting to the near-mid IR photometric points, has a dust temperature of $174 \mathrm{~K}$ at an estimated radius from the star of $13 \mathrm{au}$, assuming blackbody emission from the grains.

\subsection{Hd 170773}

HD 170773 is an F5 star at a distance of 37 pc with an estimated age of $200 \mathrm{Myr}$ (Rhee et al. 2007) but which could be as young as $150 \mathrm{Myr}$ and as old as $300 \mathrm{Myr}$ (Zuckerman \& Song 2004). The SONS survey $850 \mu \mathrm{m}$ image shows a resolved structure, well-centred on the stellar position but extending to the south-east (Fig. A11c). The peak flux is $16.5 \pm 1.8 \mathrm{mJy}^{\text {beam }}{ }^{-1}$, whilst the integrated flux, measured in an aperture of 40 arcsec in diameter, is $26.0 \pm 2.3 \mathrm{mJy}$. Assuming the structure represents a disc about the star the deconvolved radius is $6.8 \operatorname{arcsec}(\sim 250 \mathrm{au})$ at a PA of $140^{\circ}$ (roughly aligned in a south-east direction; see Table 4), compared to a radius of 73 au obtained assuming pure blackbody emission 


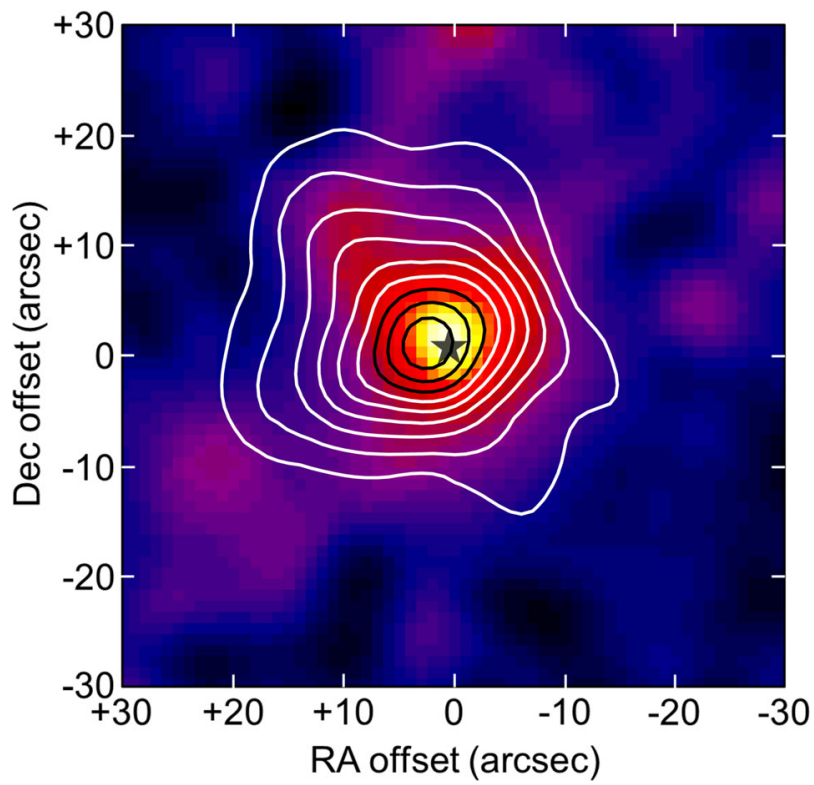

Figure 22. The $450 \mu \mathrm{m} \mathrm{S} / \mathrm{N}$ image from observations of Vega ( $\alpha$ Lyr; HD 172167) with contours from the $850 \mu \mathrm{m}$ image overlaid. The contours are scaled from $-3 \sigma$ to the maximum $\mathrm{S} / \mathrm{N}$ in the image. The contours start at $-3 \sigma$ (dashed white) and then solid colours from $4 \sigma$ to the maximum in $1.5 \sigma$ steps. The star symbol represents the position of the star with respect to the disc.

from the dust grains. The $850 \mu \mathrm{m}$ peak flux also agrees well with a previous photometry using LABOCA on APEX at $870 \mu \mathrm{m}$ of $18 \pm 5.4$ mJy beam $^{-1}$ (Liseau et al. 2010).

Herschel/PACS observations reveal a disc of radius $5.9 \operatorname{arcsec}(\sim 217 \mathrm{au})$ over the observed wavelength range of 70 $160 \mu \mathrm{m}$ (Moór et al. 2015), inclined at $31^{\circ}$ to the plane of the sky and at a PA of $118^{\circ}$ (Moór et al. 2015). These are in reasonably good agreement with the SCUBA-2 results. Modelling of the Herschel/PACS results suggested inner and outer radii for the disc of 81 and $265 \mathrm{au}$, respectively (Moór et al. 2015). The far-IR SED is well-characterized by measurements from Spitzer/MIPS (Chen et al. 2014) and Herschel PACS and SPIRE (Pawellek et al. 2014; Moór et al. 2015). The fit to the SED gives a single-component disc with a dust temperature of $46 \mathrm{~K}$, a $\beta$ value of $0.5-1.4$, and an estimated dust mass of $0.19 \mathrm{M}_{\oplus}$.

\subsection{Vega (HD 172167, $\alpha$ Lyr)}

Vega (HD 172167) is a luminous A0 star at a distance of $7.7 \mathrm{pc}$ with an estimated age of $700 \mathrm{Myr}$ with a range extending from 625 to $850 \mathrm{Myr}$ (Monnier et al. 2012). The SONS image at $850 \mu \mathrm{m}$ reveals a well-resolved, largely circularlysymmetric, smooth structure, with the peak in emission offset from the proper motion-corrected stellar position by only $2 \operatorname{arcsec}$ (Fig. A11d). The peak flux is $13.7 \pm$ $0.8 \mathrm{mJy}$ beam $^{-1}$, whilst the integrated flux, measured within a 50 arcsec diameter aperture, is $34.4 \pm 1.4 \mathrm{mJy}$. Both of these flux measurements are approximately 25 per cent lower than the original SCUBA measurements (Holland et al. 1998), and just outside the threshold of the estimated measurement errors. Emission was also detected with high $\mathrm{S} / \mathrm{N}$ at $450 \mu \mathrm{m}$ with an integrated flux density of $229 \pm 14 \mathrm{mJy}$ (Fig. 22). Interpreting the structure as a disc about the star gives deconvolved fitted radii, based on the measured 450 and $850 \mu \mathrm{m}$ images, of 9.5 and $17.5 \operatorname{arcsec}(\sim 73$ and $135 \mathrm{au})$, respectively.
The original SCUBA $850 \mu \mathrm{m}$ result suggested a more clumpy structure than seen in the new SONS images, with the main peak being offset from the star by $\sim 6$ arcsec (Holland et al. 1998). With approximately 13 years separating the observations, it is unlikely that there would be any significant change to the physical structure of the disc that might affect the offset. Both the SONS 450 and $850 \mu \mathrm{m}$ images appear to be slightly elongated in the north-east direction, and it is possible that this extension is caused by another source. Given the proper motion of the star over the period between the SCUBA and SCUBA- 2 observations $(+2,+3.6$ arcsec in RA and Dec., respectively) a possible scenario is that the north-east 'clump', seen in the original SCUBA $850 \mu \mathrm{m}$ image, is a background object (see Section 6.5). There are, however, issues with this explanation in terms of the relative brightness of the peaks, and it is perhaps more plausible that the difference is due to a combination of statistical errors and pointing shifts from the two data sets (see Section 6.4 for a further discussion of offsets).

The Vega debris disc has been extremely well-studied in the near-IR to millimetre region since the original discovery of its large IR excess by IRAS (Aumann et al. 1984). Interferometric observations at $1.3 \mathrm{~mm}$ detected dust peaks offset from the star by 8-14 arcsec that were speculated to be part of a ring of emission at a radius of 60-95 au (Koerner, Sargent \& Ostroff 2001; Wilner et al. 2002). Multiband imaging using Spitzer/MIPS in the mid-farIR revealed a resolved, smooth disc, devoid of any clumpiness ( $\mathrm{Su}$ et al. 2005). The disc also appeared to be much larger than originally thought, with measurements at $160 \mu \mathrm{m}$ suggesting a radius as large as $800 \mathrm{au}$. Further observations with the CSO at 350/450 $\mu \mathrm{m}$ appeared to show a ring-like morphology with inhomogeneous azimuthal structure, having inner and outer radii of 6.9 and 13.9 arcsec ( $\sim 53$ and $\sim 107 \mathrm{au}$ ), respectively (Marsh et al. 2006). These observations tended to support a hypothesis that the disc changes from a smooth, axisymmetric structure in the IR to a more clumpy structure in the millimetre, perhaps with an intermediate structural state governed by a grain population of around $1 \mathrm{~mm}$ in size at short submillimetre wavelengths. Theories to explain such a structure included a collision between two massive planetesimals (statistically unlikely) or that the clumps (dominated by grains of $1 \mathrm{~mm}$ and larger) are trapped in resonance with a Neptune-mass planet, whilst smaller grains are perturbed by radiation pressure and have a more uniform distribution in the disc (Wilner et al. 2002; Marsh et al. 2006; Wyatt 2006).

Herschel observations with PACS and SPIRE brought into question the clumpy nature of the disc at submillimetre wavelengths. All images between 70 and $500 \mu \mathrm{m}$ show a well-resolved, but largely smooth disc, leading to the conclusion that the disc is steady-state in nature (Sibthorpe et al. 2010). The star-subtracted Herschel images at 70 and $160 \mu \mathrm{m}$ show that the peak surface brightness of the disc occurs at a radius of $\sim 11 \operatorname{arcsec}(\sim 85 \mathrm{au})$ from the star. Furthermore, observations from SMA, CARMA and GBT at wavelengths of $870 \mu \mathrm{m}, 1.3 \mathrm{~mm}$ and $3 \mathrm{~mm}$, respectively, do not reveal any clumpy structure on the size-scale expected from previous observations (Hughes et al. 2012). Analysis of these more recent interferometric results, in particular, demonstrate that the observations are consistent with a smooth disc having an inner radius of between 20 and 100 au and a broad width estimated at $>50 \mathrm{au}$.

Modelling of the well-sampled SED of Vega suggests a twocomponent fit to the overall IR excess. The fit to the warmer, inner component is consistent with the analysis of Spitzer/IRS and $24 \mu \mathrm{m}$, and MSX mid-IR photometry, carried out by Su et al. (2013), in which the existence of a warm, unresolved 'asteroid belt' at a radius of $\sim 14$ au was postulated. The colder component is modelled 
as a disc/belt with a dust temperature $46 \mathrm{~K}$, and a $\beta$ value in the range of 1.2-1.6, with an estimated dust mass of $0.011 \mathrm{M}_{\oplus}$ derived from the $850 \mu \mathrm{m}$ flux. The derived dust temperature for the cold disc is also in good agreement with previous estimates ( $\mathrm{Su}$ et al. 2005). The relatively high $\beta$ range means that the equivalent disc radius, assuming blackbody emission, is $260 \mathrm{au}$, this being significantly lower than the values measured from the images. Since the measured disc radius at $450 \mu \mathrm{m}$ is also less than that at $850 \mu \mathrm{m}$ by almost a factor of 2, one possibility to explain this discrepancy is a lack of sensitivity in the SONS observations to low-level, extended emission, i.e. the overall emission extends well beyond the lowest contours of the current maps, possibly to several hundred au. This is further discussed in Section 6.2.

The overall conclusion, supported by the new SONS images, is that the Vega debris disc is largely smooth and circularly symmetric at IR to millimetre wavelengths. Vega is, however, certainly a complex system, most likely containing an inner 'asteroid belt' analogue at a radius of $<14 \mathrm{au}$, in addition to a smooth, wide outer disc, perhaps containing another belt at 30-100 au, as well as a diffuse halo extending to many hundreds of au.

\subsection{Hd 181327}

HD 181327 is an F5 star and a member of the $\beta$ Pictoris moving group, at a distance of $52 \mathrm{pc}$ with an estimated age of $23 \pm 3$ Myr (Mamajek \& Bell 2014). At $850 \mu \mathrm{m}$ the SONS image shows a bright, unresolved peak, well-centred on the stellar position with a flux density of $23.6 \pm 3.4 \mathrm{mJy}$ (Fig. A12a). The relatively high noise level is due to the short integration time $(1 \mathrm{~h})$ and the low declination of the source. Interpreted as a disc about the star, the upper limit to the radius from the $850 \mu \mathrm{m}$ image is $390 \mathrm{au}$. The LABOCA camera on APEX measured a peak flux of $24.2 \mathrm{mJy}$ beam $^{-1}$ at $870 \mu \mathrm{m}$ (Nilsson et al. 2009), consistent with the $850 \mu \mathrm{m}$ SONS result. The integrated flux from the LABOCA measurement, however, is far higher at $51.7 \pm 6.2 \mathrm{mJy}$, which suggests that the disc is well resolved. This is contrary to the result from the SONS $850 \mu \mathrm{m}$ image.

The debris disc around HD 181327 has been well-studied in the IR through to the millimetre. The disc was first resolved by HST/NICMOS and modelling revealed a face-on ring peaking in intensity at a radius of $1.7 \operatorname{arcsec}(\sim 86 \mathrm{au})$ with a width of $0.7 \operatorname{arcsec}(\sim 36 \mathrm{au})$, inclined at $32^{\circ}$ to the plane of the sky and at a PA of $107^{\circ}$ (Schneider et al. 2006; Stark et al. 2014). More recently, the disc has been resolved using ALMA at $1.3 \mathrm{~mm}$ revealing a circular ring with the emission radially confined between 1.0 and $2.4 \operatorname{arcsec}(\sim 50-125 \mathrm{au})$ with a peak intensity occurring at a radius of $1.7 \operatorname{arcsec}(\sim 86 \mathrm{au})$ from the star at a PA of $99^{\circ}$ (Marino et al. 2016). The ALMA observations also detected ${ }^{12} \mathrm{CO}(2-1)$ emission around an $\mathrm{F}$ star for the first time.

Both the HST/NICMOS and ALMA results suggest the existence of one or more compact, background objects that could have contaminated the LABOCA photometry, leading to an artificially high flux estimate for the disc. There are also some low-level $(<3 \sigma)$ features in the vicinity of the disc in the SONS $850 \mu \mathrm{m}$ image, which could provide extra evidence to support this hypothesis. For the modelling of the SED it is therefore assumed that the peak flux value from LABOCA of $24.2 \mathrm{mJy}^{\text {beam }}{ }^{-1}$ at $870 \mu \mathrm{m}$ is more representative of a true disc flux (although the SED in Fig. A11 a shows the integrated flux measurement this is not used in the fit). The SED is modelled as a two-component disc with the warmer element having a dust temperature of $95 \mathrm{~K}$ and located at a radius of $16 \mathrm{au}$ from the star (assuming the grains behave like perfect blackbod- ies). Photometry from Spitzer/MIPS at 24, 70 and $160 \mu \mathrm{m}$ (Chen, Fitzgerald \& Smith 2008; Chen et al. 2014), and Herschel/PACS observations (archival data) help constrain the mid-far-IR part of the SED, in addition to a $7 \mathrm{~mm}$ photometric point from the VLA MacGregor et al. (2016a). Fitting the SED submillimetre points using the SONS result at $850 \mu \mathrm{m}$ suggests a cold disc component with a dust temperature of $63 \mathrm{~K}$, a $\beta$ value tightly constrained between 0.4 and 0.6 , and an estimated dust mass of $0.25 \mathrm{M}_{\oplus}$. The total flux measured of $7.9 \pm 0.2 \mathrm{mJy}$, measured by ALMA at $1.3 \mathrm{~mm}$, is also consistent with the fit to the SED (Marino et al. 2016).

\subsection{Hd 182681}

HD 182681 is a luminous B8 star at a distance of $70 \mathrm{pc}$ with an estimated age of $145 \mathrm{Myr}$ (Chen et al. 2014), but is sometimes referred to as a pre-main-sequence star with an age as young as $19 \mathrm{Myr}$, but also as old as $208 \mathrm{Myr}$ (Moór et al. 2015). The $850 \mu \mathrm{m}$ image shows an unresolved disc, just offset from the stellar position, with a flux density of $6.8 \pm 1.2 \mathrm{mJy}$ (Fig. A12b). There is a second source offset by 19 arcsec to the south-east, which is likely to be a background object, although there is a low-level ( $<3 \sigma$ significance) ridge running approximately north-west to south-east. Assuming that only the central peak is part of a disc structure, the upper limit to the disc radius from the $850 \mu \mathrm{m}$ image is $525 \mathrm{au}$.

The disc was resolved by Herschel/PACS with the $160 \mu \mathrm{m}$ image indicating a disc of radius $3.3 \operatorname{arcsec}(\sim 230 \mathrm{au})$, inclined at $67^{\circ}$ to the line-of-sight and at a PA of $56^{\circ}$ (Moór et al. 2015). Modelling of the PACS images suggests a structure where the flux density peaks at $159 \mathrm{au}$, with inner and outer radii of 52 and 263 au, respectively. The south-east peak is not visible in any of the Herschel images, including longer wavelength results from SPIRE at 350 and $450 \mu \mathrm{m}$. Photometry from Herschel also helps to constrain the mid-far-IR part of the SED (Pawellek et al. 2014; Moór et al. 2015). Fitting the SED suggests a single-component disc with a dust temperature of $80 \mathrm{~K}$, a $\beta$ value in the range of $0.2-1.0$ and an estimated dust mass of $0.104 \mathrm{M}_{\oplus}$.

\subsection{Hd 191089}

HD 191089 is an F5 star and a member of the $\beta$ Pictoris moving group. It lies at a distance of $52 \mathrm{pc}$ with an estimated age of $23 \mathrm{Myr}$ (Mamajek \& Bell 2014). The SONS survey image at $850 \mu \mathrm{m}$ reveals a compact, unresolved structure, with a flux density of $4.9 \pm 0.9 \mathrm{mJy}$ (Fig. A12c). The peak emission is offset from the star position by $\sim 6$ arcsec, i.e. greater than the maximum offset of 3.5 arcsec, caused by statistical uncertainties and expected pointing errors (see Section 6.4 for a discussion of offsets). Based on other observations (see below), it is believed that the observed structure is indeed a disc associated with the star, but there exists the possibility that there could be some contamination from a background source to account for the offset (see Section 6.5). The upper limit to the disc radius from the observed $850 \mu \mathrm{m}$ image is $395 \mathrm{au}$.

The disc has been resolved from observations made with the T-ReCS instrument on the Gemini South telescope at $18.3 \mu \mathrm{m}$, which show the structure extending to a maximum radius of 0.85 $\operatorname{arcsec}(\sim 90 \mathrm{au})$ at PA of $80^{\circ}$ (Churcher, Wyatt \& Smith 2011). Modelling indicates that the emission arises from a dust belt with radius $28-90$ au from the star, inclined at $35^{\circ}$ to edge on, and with a central cavity largely devoid of emission. The disc has also been imaged in scattered light using the HST/NICMOS, revealing a ringlike structure that peaks in scattered light at $\sim 73 \mathrm{au}$, consistent with the mid-IR image (Soummer et al. 2014). The far-IR part of 


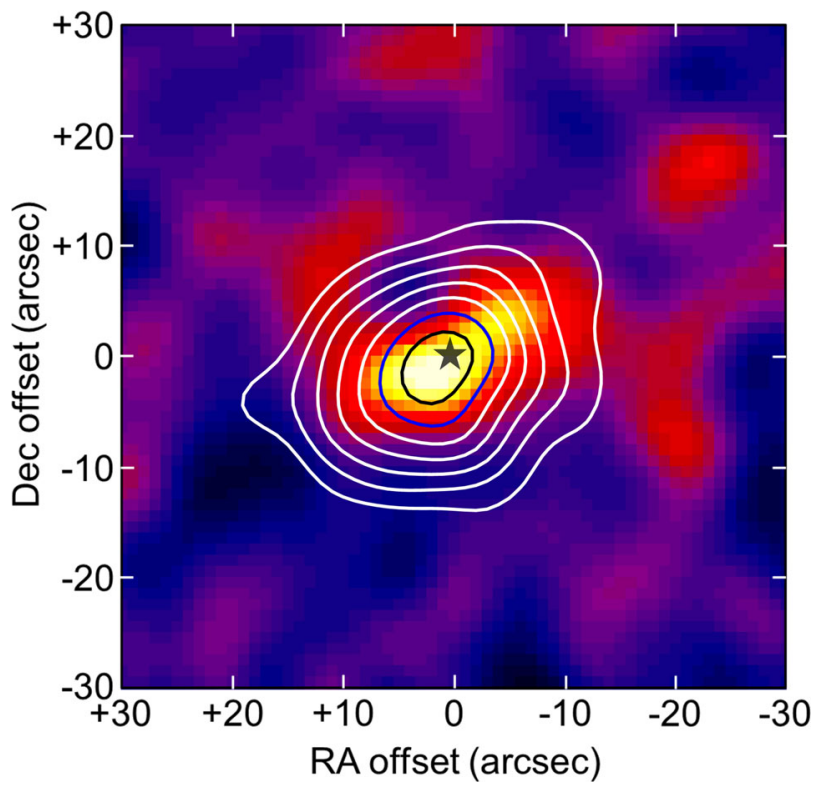

Figure 23. The $450 \mu \mathrm{m} \mathrm{S} / \mathrm{N}$ image from observations of HD 197481 (AU Mic) with contours from the $850 \mu \mathrm{m}$ image overlaid. The colours are scaled from $-3 \sigma$ to the maximum $\mathrm{S} / \mathrm{N}$ in the image. The contours start at $-3 \sigma$ (dashed white) and then solid colours from $4 \sigma$ to the maximum in $2 \sigma$ steps. The star symbol represents the position of the star with respect to the disc.

the SED is well characterized by measurements from Spitzer/MIPS (Chen et al. 2014), the CSO SHARC-II instrument (Roccatagliata et al. 2009) and Herschel PACS and SPIRE (archival data). The fit to the SED gives a single-component disc with a dust temperature of $89 \mathrm{~K}$, a $\beta$ value in the range $0.3-2.3$ and an estimated dust mass of $0.037 \mathrm{M}_{\oplus}$.

\subsection{AU Microscopii (HD 197481)}

AU Mic (HD 197481) is a young M1 star and a member of the $\beta$ Pictoris moving group, at a distance of $10 \mathrm{pc}$ with an estimated age of 23 Myr (Mamajek \& Bell 2014). The disc appears to be unresolved at $850 \mu \mathrm{m}$ with a flux density of $12.9 \pm 0.8 \mathrm{mJy}$ (Fig. A12d), despite apparently showing a slight extension roughly in the northwest to south-east direction (Matthews et al. 2015). Indeed, this extension is confirmed with $450 \mu \mathrm{m}$ observations that reveal a disc of deconvolved radius $7.1 \operatorname{arcsec}\left(\sim 70 \mathrm{au}\right.$ ) at a PA of $\sim 127^{\circ}$ (as shown in Fig. 23, with details of the disc size given in Table 4). The $450 \mu \mathrm{m}$ integrated flux, measured within a 40 arcsec diameter aperture centred on the star, is $57 \pm 9 \mathrm{mJy}$, consistent with previous photometry using JCMT/SCUBA at 450 and $850 \mu \mathrm{m}$ (Liu et al. 2004) and CSO/SHARC-II at $350 \mu \mathrm{m}$ (Chen et al. 2005).

The far-IR excess from AU Mic was first detected by IRAS (Moshir et al. 1990), and since then it has been studied across the spectrum from the optical to millimetre, with much of the interest focusing on the fact that it was the first M star to show evidence of a debris disc. Scattered light images of the disc from HST/ACS, the second of which to be resolved at optical wavelengths, revealed an edge-on disc extending to a radius of $10 \operatorname{arcsec}(\sim 100 \mathrm{au})$ at a PA of $128^{\circ}$ (Krist, Ardila \& Golimowski 2005). The disc was also resolved from Herschel/PACS observations at 70 and $160 \mu \mathrm{m}$, confirming the wide edge-on belt extending roughly from 1 to 4 arcsec $(\sim 9-40 \mathrm{au})$ at a PA of $135^{\circ}$ (Matthews et al. 2015), somewhat less in size than the SONS $450 \mu \mathrm{m}$ image shows. The HST results, and those from Herschel at $70 \mu \mathrm{m}$, suggest the presence of an extra component of small grains (sometimes referred to as a 'halo'), extending beyond the radius of the main AU Mic belt, possibly to a radius of 140 au (Matthews et al. 2015).

The disc was first resolved at millimetre wavelengths by the SMA, revealing the broad belt with the emission peaking at a radius of $3.5 \operatorname{arcsec}(\sim 35 \mathrm{au})$ at a PA of $130^{\circ}$ (Wilner et al. 2012). Subsequent imaging with ALMA at $1.3 \mathrm{~mm}$ shows a 10:1 aspect ratio for the edge-on belt, extending from a radius of $0.9 \operatorname{arcsec}(\sim 9 \mathrm{au})$ outwards to $4 \operatorname{arcsec}(\sim 40 \mathrm{au})$, at a PA of $128^{\circ}$ (MacGregor et al. 2013) in agreement with the Herschel results. The ALMA image was modelled as a narrow 'birth-ring' or 'parent belt' of planetesimals at 40 au (MacGregor et al. 2013). The SED is well-characterized in the far-IR with additional photometry from Herschel/SPIRE (Matthews et al. 2015), and is fit by an almost pure blackbody spectrum $(\beta=$ $0-0.3$; see Section 6.3 for further discussion) at a dust temperature of $50 \mathrm{~K}$, and a estimated dust mass of $6 \times 10^{-3} \mathrm{M}_{\oplus}$. The radius of the disc determined from the fit to the SED, and assuming perfect blackbody emission from the dust grains, is 9 au, consistent with the inner edge estimates from ALMA imaging.

\subsection{Hd 205674}

HD 205674 is an F0 star and possible member of the AB Doradus moving group lying at a distance of $52 \mathrm{pc}$ with an estimated age of $130 \mathrm{Myr}$ in a range of 110-150 Myr (Barrado y Navascués et al. 2004). The image at $850 \mu \mathrm{m}$ shows that the the emission is largely concentrated in two peaks running in a line from north to south (Fig. A13a). The image presented in this paper benefits from a considerable increase in integration time over that published in Panić et al. (2013), which clearly showed a peak to the south offset by 10 arcsec from the star. The main dust peak has a flux of $4.0 \pm 0.7 \mathrm{mJy}$ beam $^{-1}$, and is offset by $\sim 6$ arcsec from the stellar position, greater than the maximum offset of 3.2 arcsec expected due to statistical uncertainties and pointing errors (see Section 6.4). The total flux, including the second peak to the south, and measured in a 40 arcsec diameter aperture centred between the two peaks, is $7.5 \pm 1.1 \mathrm{mJy}$. Both the offset of the main peak from the star and the elongated structure are difficult to explain. The observed structure certainly hints at two distinct peaks, perhaps suggesting that the peak to the south could be a background source.

Herschel/PACS imaging at 100 and $160 \mu \mathrm{m}$ reveals an emission peak that is well-centred on the star position, and does not show any evidence of an extension or second source to the south (see Fig. 24). This strengthens the argument that the the peak seen to the south in the SONS $850 \mu \mathrm{m}$ image is most likely a background source, although no such source has been identified from catalogues. Hence, in this paper the emission surrounding HD 205674 is interpreted as an offset disc, assuming that the southern peak is a background source. The far-IR SED is well constrained by measurements from Spitzer/MIPS (Moór et al. 2011b) and archival data from Herschel PACS and SPIRE but the $850 \mu \mathrm{m}$ SONS flux is the only other long wavelength photometric data. Fitting the SED suggests a disc with a dust temperature of $60 \mathrm{~K}, \beta$ in the range $0.1-1.1$ and an estimated dust mass of $0.044 \mathrm{M}_{\oplus}$.

\subsection{Hd 206893}

HD 206893 is an F5 star, lying at a distance of 38 pc with an age estimated to be $860 \mathrm{Myr}$ (Pace 2013), but could be as young as 200 Myr (Zuckerman \& Song 2004) or as old as 2.1 Gyr (David $\&$ Hillenbrand 2015). The SONS image at $850 \mu \mathrm{m}$ shows a faint, 


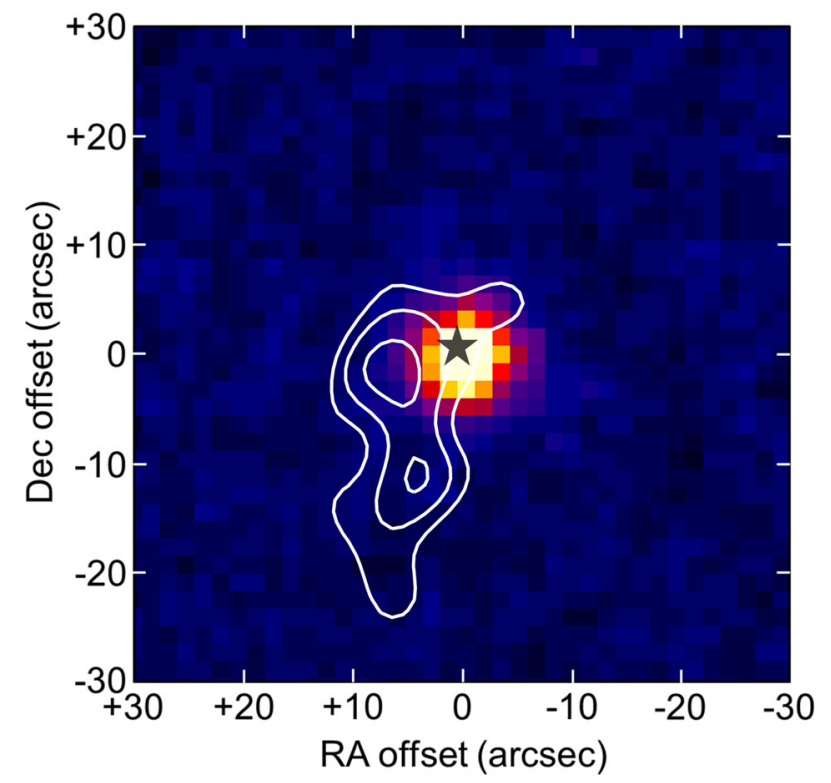

Figure 24. Herschel/PACS $100 \mu \mathrm{m}$ image of HD 205674 with the SONS survey $850 \mu \mathrm{m}$ contours overlaid. The contours start at $3 \sigma$ and increase in $1 \sigma$ steps. The Herschel image is taken from the Herschel Science Archive.

unresolved peak of flux density $4.5 \pm 1.1 \mathrm{mJy}$, offset from the stellar position by 4 arcsec (Fig. A13b). Interpreting the peak as an unresolved disc associated with the star, gives an upper limit for the disc radius of $290 \mathrm{au}$.

Spitzer detected excess flux from the star at wavelengths between 3.6 and $160 \mu \mathrm{m}$ (Moór et al. 2006). The disc has also been marginally resolved by Herschel/PACS observations at $70 \mu \mathrm{m}$, revealing a dust ring extending from 1.3 to $5.2 \operatorname{arcsec}(\sim 50-200 \mathrm{au})$ at a PA of $60^{\circ}$ (Milli et al. 2017). The disc has also been imaged in scattered light extending to a radius of $4-5 \operatorname{arcsec}(\sim 150-190 \mathrm{au})$ at a very similar PA (Milli et al. 2017). Recently, a low-mass companion brown-dwarf (mass in the range 24-73 $M_{\text {Jup }}$ ) was also detected using VLT/SPHERE at H-band, lying in an orbit of radius $10 \mathrm{au}$ from HD 206893 (Milli et al. 2017). The detection of a low-mass companion inside a massive debris disc makes this system analogous to other young planetary systems, such as $\beta$ Pictoris and HR 8799.

The SED is reasonably well-sampled in the near-far-IR, but only has the SONS $850 \mu \mathrm{m}$ photometric point at long wavelengths. The SED is modelled with a single-temperature modified blackbody deriving a dust temperature of $54 \mathrm{~K}$, a $\beta$ value only loosely constrained between values of 0.5 and 2.7 and an estimated dust mass of $0.030 \mathrm{M}_{\oplus}$ derived from the $850 \mu \mathrm{m}$ flux.

\subsection{Hd 207129}

HD 207129 is a Solar analogue (G2 star) at a distance of 16 pc with an estimated age of $3800 \mathrm{Myr}$ but with large uncertainties spanning the range of 1300-7400 Myr (Holmberg, Nordström \& Anderson 2007). The image at $850 \mu \mathrm{m}$ shows emission in two $4 \sigma-5 \sigma$ peaks with the star equidistant between the two. The peak to the west has a flux of $7.0 \pm 1.2 \mathrm{mJy}$ beam $^{-1}$ (Fig. A13c), whilst the integrated flux within a 40 arcsec diameter aperture, covering both peaks, is $10.8 \pm 1.8 \mathrm{mJy}$, indicating the disc is marginally resolved by the JCMT beam. The symmetric nature of the peaks about the star suggests a possible inclined toroidal geometry for the structure (perhaps similar to Fomalhaut, as described in Section 3.3). Interpreting the structure as a disc, extended in a roughly east-west direction, gives a deconvolved radius of $10 \operatorname{arcsec}(\sim 160 \mathrm{au})$ at a PA angle of $\sim 115^{\circ}$.

Being a relatively close solar analogue, HD 207129 has been wellstudied at most wavelengths between the optical and millimetre. The debris disc was first discovered by IRAS (Walker \& Wolstencroft 1988) with subsequent far-IR photometry from ISO (Jourdain de Muizon et al. 1999) and Spitzer (Krist et al. 2010). The disc is also well resolved in scattered light observations revealing a narrow ring of radius $10 \operatorname{arcsec}(\sim 160 \mathrm{au})$ at a PA of $127^{\circ}$ (Krist et al. 2010). The HD 207129 disc has been resolved by Herschel/PACS as part of the DUNES key programme (Marshall et al. 2011; Löhne et al. 2012). Modelling of the $100 \mu \mathrm{m}$ PACS image suggests a ring of radius $8.7 \operatorname{arcsec}(\sim 140 \mathrm{au})$, consistent with an interpretation of collisional dust being produced in an icy 'exo-Kuiper' belt. The disc appears inclined at $53^{\circ}$ from pole-on with a PA of $122^{\circ}$. The SCUBA-2 image at $850 \mu \mathrm{m}$ is in good agreement with both the scattered light and the Herschel results. The SED is well-sampled by Spitzer/MIPS (Trilling et al. 2008; Tanner et al. 2009; Krist et al. 2010) and Herschel PACS and SPIRE (Marshall et al. 2011; Eiroa et al. 2013) in the far-IR. Fitting the SED suggests a disc with a single dust temperature of $46 \mathrm{~K}$, a $\beta$ value of $0.4-1.1$, and an estimated dust mass of $0.015 \mathrm{M}_{\oplus}$.

\subsection{Hd 212695}

HD 212695 is an old F5 star at a distance of $47 \mathrm{pc}$ with an estimated age of $2300 \mathrm{Myr}$ (Trilling et al. 2007). The $850 \mu \mathrm{m}$ image shows unresolved emission, just offset from the star, with a flux density of $5.7 \pm 1.1 \mathrm{mJy}$ (Fig. A13d). Interpreting this structure as a disc about the star gives an upper limit to the disc radius from the $850 \mu \mathrm{m}$ image of $350 \mathrm{au}$. The SED is not well-constrained in the far-IR, only having photometry from Spitzer/MIPS at $70 \mu \mathrm{m}$ (Chen et al. 2014). A fit to this limited SED suggests that the single-component disc is cold with a dust temperature of $35 \mathrm{~K}$ (but with errors of $\pm 19 \mathrm{~K}$ ) and an estimated dust mass of $0.086 \mathrm{M}_{\oplus}$.

\subsection{Pegasi (HD 213617)}

$39 \mathrm{Peg}$ is a F1 star at a distance of $50 \mathrm{pc}$ with an estimated age of $1200 \pm 300$ Myr (Moór et al. 2011b). The SONS image at $850 \mu \mathrm{m}$ shows a marginallydetected, unresolved peak of flux density $4.6 \pm$ $1.3 \mathrm{mJy}$, offset from the stellar position by 4 arcsec (Fig. A14a). Interpreting the peak as an unresolved disc associated with the star gives an upper limit for the disc radius of $380 \mathrm{au}$.

Spitzer detected excess flux from the star at wavelengths up to $70 \mu \mathrm{m}$ (Moór et al. 2011b), but the SONS $850 \mu \mathrm{m}$ photometry is the only data at longer wavelengths. Modelling of the SED suggests a single-component fit having a dust temperature of $59 \mathrm{~K}$, a $\beta$ value only loosely constrained between values of 0 and 2.1 , and an estimated dust mass of $0.049 \mathrm{M}_{\oplus}$ derived from the $850 \mu \mathrm{m}$ flux.

\subsection{Fomalhaut (HD 216956, $\alpha$ PsA)}

Fomalhaut (HD 216956) is a luminous A4 star at a distance of $7.7 \mathrm{pc}$ with an estimated age of $440 \mathrm{Myr}$ of ( $\pm 40 \mathrm{Myr}$ ) (Mamajek \& Bell 2014). The SCUBA-2 $850 \mu \mathrm{m}$ image shows the familiar doublelobed disc structure at a PA of $160^{\circ}$, with the star positioned equidistant between the lobes (Fig. A14b). The two peaks have fluxes of $26.3 \pm 1.0 \mathrm{mJy}$ beam $^{-1}$ (north-west) and $25.2 \pm 1.0 \mathrm{mJy} \mathrm{beam}^{-1}$ (south-east), in good agreement with previous submillimetre results (Holland et al. 1998, 2003). The integrated flux within a 60 arcsec 
diameter aperture is $91.2 \pm 2.5 \mathrm{mJy},{ }^{4}$ also in good agreement with the previous estimates (Section 3.3). As previously discussed in Section 4.3 the emission morphology is not well-represented by a Gaussian profile, and the resultant model-subtracted map shows a residual peak at the position. Nevertheless, for this paper such an approximation gives a reasonable estimation for the overall size of the structure. Interpreted as a disc/ring about the star, the deconvolved major and minor radii from the $2 \mathrm{D}$ Gaussian fitting are 19.7 and $7.5 \operatorname{arcsec}(\sim 151$ and $\sim 57 \mathrm{au})$, respectively.

The IR excess from Fomalhaut was first discovered and resolved by IRAS (Gillet 1986; Backman \& Paresce 1993). Further indication that the excess could be an extended disc structure came from pointby-point photometry using the JCMT (Zuckerman \& Beckin 1993). In the late 1990s, the SCUBA camera (Holland et al. 1998) provided the first true image of the thermal emission from the Fomalhaut debris disc, resolving the double-lobed structure. The SCUBA image suggested a massive torus-like Kuiper Belt surrounds the star, with the possibility of one or more planets acting as shepherds to the disc structure. The first optical observations of the disc in scattered light revealed a narrow elliptical belt with semimajor and minor axes of 18.5 and 7.6 arcsec $(\sim 141$ and $\sim 58 \mathrm{au})$, respectively (Kalas, Graham \& Clampin 2005), at a PA of $156^{\circ}$ and with an estimated thickness of $15 \mathrm{au}$. Modelling of the geometry of the dust belt concluded that the observed features, such as the sharp inner edge and variation in the azimuthal brightness, were likely attributable to a planetary system.

The discovery of the first planet in the system, Fomalhaut b, was also made via $H S T$ observations with a location just inside the inner radius of the ring (Kalas et al. 2008), and subsequent observations have provided further insights on both the main belt structure and the possible planetary system (Kalas et al. 2013). However, the orbit of Fomalhaut b is not apsidally aligned with the dust ring, and so it may be the case that additional planets are responsible for shaping the dust morphology. It has also been suggested that Fomalhaut $b$ is not actually a planet, but either a dust cloud (perhaps orbiting a planet) (Kennedy \& Wyatt 2011) or the dusty aftermath of a collision between two Kuiper belt-like objects (Galicher et al. 2013).

Herschel observations provided high dynamic range images of the dust belt in the far-IR, as well as evidence that the system is remarkably active with dust grains being produced at a very high rate by a collisional cascade of planetesimals (Acke et al. 2012). Modelling of the $70 \mu \mathrm{m}$ PACS image showed a ring with a mean radius of $18 \operatorname{arcsec}(\sim 138$ au $)$ at a $\mathrm{PA}$ of $157^{\circ}$, consistent with the submillimetre and optical images. Further constraints on the Fomalhaut planetary system have been obtained by high angular resolution observations with ALMA of the north-west side of the belt. Observations at $850 \mu \mathrm{m}$ with $\sim 1.5$ arcsec resolution show a ring that peaks in surface brightness at a radius of 18.4 arcsec $(\sim 142 \mathrm{au})$, with a PA of $168^{\circ}$. The ring is inclined to the plane of the sky by $66^{\circ}$ and has a width of $\sim 17$ au (Boley et al. 2012). Moreover, the image showed that almost all of the emission is confined to the ring, with the flux estimates also agreeing with previous submillimetre measurements at $850 \mu \mathrm{m}$ (for the half of the ring that was observed). More recent observations with ALMA on the entire ring at $1.3 \mathrm{~mm}$, tightly constrain the radius to $\sim 136$ au with a width of 13.5 au (MacGregor et al. 2017; White et al. 2017).

The Fomalhaut disc was also detected with high $\mathrm{S} / \mathrm{N}$ at $450 \mu \mathrm{m}$, and Fig. 25 shows the $450 \mu \mathrm{m}$ S/N image with the $850 \mu \mathrm{m}$ con-

\footnotetext{
4 The calibration uncertainty at $850 \mu \mathrm{m}$ adds an additional 7 per cent to this
} error estimate.

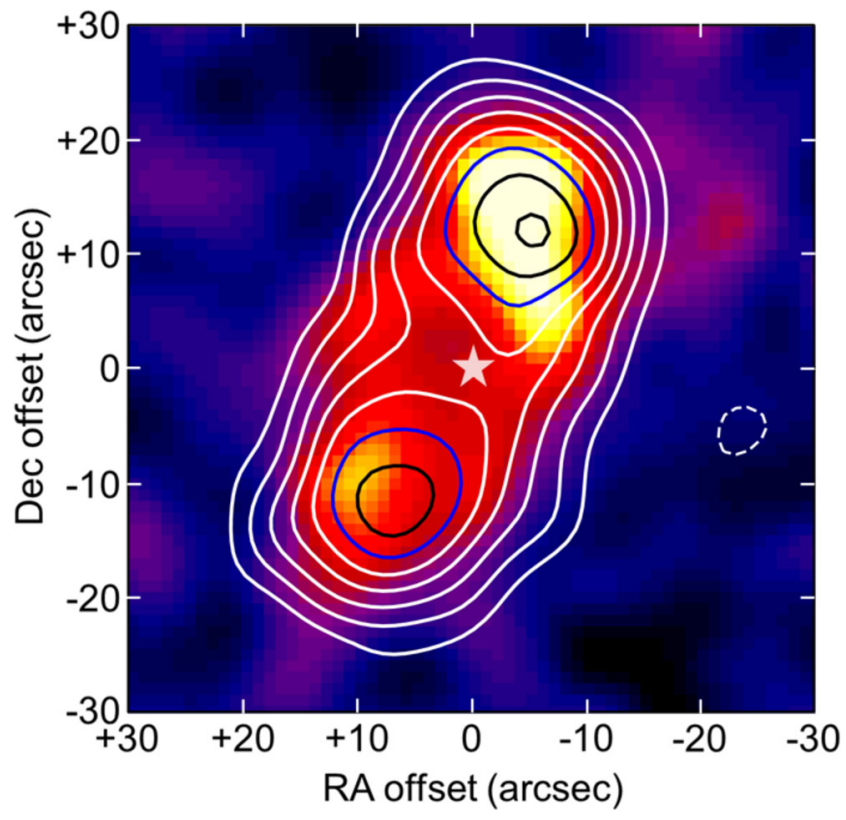

Figure 25. The $450 \mu \mathrm{m} \mathrm{S} / \mathrm{N}$ image from observations of Fomalhaut (HD 216956) with contours from the $850 \mu \mathrm{m}$ image overlaid. The colours are scaled from $-3 \sigma$ to the maximum $\mathrm{S} / \mathrm{N}$ in the image. The contours start at $-5 \sigma$ (dashed white) and then solid colours from $5 \sigma$ to the maximum in $5 \sigma$ steps. The star symbol represents the position of the star with respect to the disc.

tours overlaid. The general morphology is consistent with previous SCUBA observations, and there is even evidence of a slight flux excess to the east (e.g. a flux difference of $10 \mathrm{mJy}$ compared to that at the equivalent radius from the star on the west side) as reported in the previous asymmetric description of the disc about the star (Holland et al. 2003). The striking difference here, however, is that the new image appears to show a brightness asymmetry between the north-west and south-east lobes at approximately the $3 \sigma$ level. This asymmetry is also supported by CSO observations of the Fomalhaut disc (Marsh et al. 2005). The theory of 'apocentre glow' predicts that a steady-state disc will show an overdensity of dust at apocentre, due to the Keplerian orbital velocity in an eccentric disc being slower at apocentre rather than at pericentre (Pan, Nesvold \& Kuchner 2016). The most recent results from high-resolution observations with ALMA provide conclusive evidence that the apocentre (north-west peak) has excess submillimetre/millimetre dust (MacGregor et al. 2017; Matrà et al. 2017). The SED in the far-IR and submillimetre is extremely well-sampled with a fit suggesting a dominant cold component disc with a dust temperature of $41 \mathrm{~K}$, a $\beta$ value well-constrained in the range of 1.0-1.3 and an estimated dust mass of $0.032 \mathrm{M}_{\oplus}$.

\subsection{HR 8799 (HD 218396)}

HR 8799 (HD 218395) is a young luminous A5 star in the Columba Moving Group (Doyon et al. 2010; Zuckerman et al. 2011), lying at a distance of $39 \mathrm{pc}$ with an estimated age of $30 \mathrm{Myr}$ (Torres et al. 2008; Marois et al. 2010). The SONS survey image at $850 \mu \mathrm{m}$ reveals a significantly extended structure, with a peak flux of $10.9 \pm 1.0 \mathrm{mJy}$ beam $^{-1}$, well-centred on the stellar position (Fig. A14c). The integrated flux, measured in a 60 arcsec diameter aperture, is $23.0 \pm 2.0 \mathrm{mJy}$, which takes in all the structure including the extension (possibly a separate background source) to 
the north-west (see discussion below). The peak flux is also consistent with the previous SCUBA flux estimate of $10.3 \pm 1.8 \mathrm{mJy}$ (Williams \& Andrews 2006), which was measured in photometry mode and so not sensitive to any extended structure. Fitting the radial extent, but without including the north-west peak, gives a deconvolved disc radius of $10 \operatorname{arcsec}(\sim 395 \mathrm{au})$ at a PA of $71^{\circ}$ (see Table 4).

The HR 8799 system also contains at least four known giant planets, all of which have orbital radii within $\sim 70$ au of the star (Marois et al. 2008, 2010). The thermal emission from the debris disc was first detected by IRAS (Sadakane \& Nishida 1986). The disc was first resolved by Spitzer at 24 and $70 \mu \mathrm{m}$ and the results of SED modelling suggested there were three distinct components to the debris system ( $\mathrm{Su}$ et al. 2009). The first is a warm $(\sim 150 \mathrm{~K})$ dust cloud orbiting within planets 'd' and 'e' at a radius of $<0.6 \operatorname{arcsec}(\sim 24 \mathrm{au})$ from the star. The second is a broad zone of cold $(\sim 45 \mathrm{~K})$ dust with a sharp inner edge and orbiting just outside the outermost planet ('planet b') at a radius of $2.3 \operatorname{arcsec}(\sim 90 \mathrm{au})$ but extending out to $7.7 \operatorname{arcsec}(\sim 300 \mathrm{au})$. The final component is a halo of small grains, perhaps originating in the cold belt, but extending from 7.7 arcsec $(\sim 300 \mathrm{au})$ to at least a radius of 25 arc$\sec (\sim 1000 \mathrm{au})$, and suggesting dust grains are prevalent at very large radii from the host star ( $\mathrm{Su}$ et al. 2009). Subsequent observations by Herschel also resolved the disc between wavelengths of 70 and $250 \mu \mathrm{m}$ (Matthews et al. 2014) with deconvolved radii of 7.2 and $7.7 \operatorname{arcsec}(\sim 284$ and $300 \mathrm{au})$ at a PA of $62^{\circ}$, based on the $70-160 \mu \mathrm{m}$ PACS results. Modelling the images also supported the Spitzer findings that the disc consists of three distinct components: a warm inner asteroid belt analogue, a planetesimal belt extending from 100 to $310 \mathrm{au}$ and an outer halo reaching a radius of $1500 \mathrm{au}$ from the star. Both the Spitzer and Herschel observations also show a compact source some 15 arcsec to the north-west, coincident with the extension seen in the $850 \mu \mathrm{m}$ image. It is therefore likely that this source is a separate background object.

The HR 8799 disc has also recently been observed with ALMA at $1.3 \mathrm{~mm}$, resolving the planetesimal belt. The emission was modelled as a broad ring at a radius of between 3.7 and $10.7 \operatorname{arcsec}(\sim 145$ and $429 \mathrm{au}$ ), with a PA of $51^{\circ}$ (Booth et al. 2016). The outermost planet in the system ('planet b') was expected to be responsible for shaping the inner edge of the belt, but the size of the belt seems inconsistent with the planet's orbit. This inconsistency suggests that either the orbit has varied over time or there is another (smaller) planet further out from the star. The measured flux density of $2.8 \mathrm{mJy}$ at $1.3 \mathrm{~mm}$ also seems inconsistent with the well-sampled SED, suggesting that the ALMA data are missing significant flux on a scale larger than its primary beam.

The SED in the far-IR is well-sampled, including the aforementioned photometry from Spitzer and Herschel. The disc is also detected with SCUBA-2 at $450 \mu \mathrm{m}$ with a peak flux of $122 \pm$ $22 \mathrm{mJy}$ beam $^{-1}(\sim 5 \sigma)$ and Fig. 26 shows the $450 \mu \mathrm{m}$ image with the $850 \mu \mathrm{m}$ contours overlaid. In common to the other (mainly shorter wavelength) observations from Herschel, there appears to be significant low-level extended emission seen to the south and south-west at $450 \mu \mathrm{m}$, outside of the reasonably compact central source. This wide extent is reflected in an integrated flux of $346 \pm 34 \mathrm{mJy}$, measured in a 60 arcsec diameter aperture centred on the star. This emission is likely due to a known dust cloud in the vicinity of HR 8799 (Matthews et al. 2014). Hence, taking this cloud into account, the peak flux value has been adopted as the photometric point at $450 \mu \mathrm{m}$. At $850 \mu \mathrm{m}$, the flux of the background source to the north-west (5.6 $\mathrm{mJy}$ ) has been removed from the total flux estimate (revised value is $17.4 \pm 1.5 \mathrm{mJy}$ ), and it has been assumed that there is no

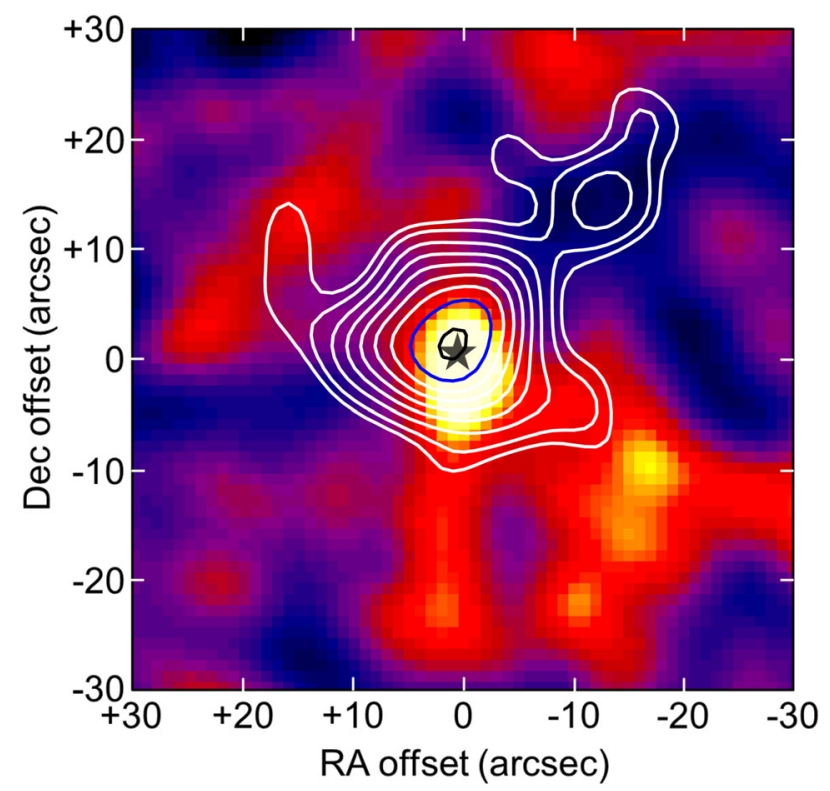

Figure 26. The $450 \mu \mathrm{m} \mathrm{S} / \mathrm{N}$ image from observations of HR 8799 (HD 218396) with contours from the $850 \mu \mathrm{m}$ image overlaid. The colours are scaled from $-3 \sigma$ to the maximum $\mathrm{S} / \mathrm{N}$ in the image. The contours start at $-3 \sigma$ (dashed white) and then solid colours from $4 \sigma$ to the maximum in $2 \sigma$ steps. The star symbol represents the position of the star with respect to the disc.

contribution from the local dust cloud at this wavelength. Based on the modelling of the SED, there appears to be evidence of two components, and fitting to the near to mid-IR photometry suggests an inner disc of $190 \mathrm{~K}$ at a radius of $5 \mathrm{au}$ from the star. The colder component, representative of the planetesimal disc, has a dust temperature of $43 \mathrm{~K}$, a $\beta$ value in the range $0.6-1.9$, and an estimated dust mass of $0.156 \mathrm{M}_{\oplus}$. Assuming the dust grains to be pure blackbodies implies that the disc would be at a radius of 80 au from the star.

\section{DISCUSSION}

\subsection{Detection rate}

The detection rate for discs at $850 \mu \mathrm{m}(3 \sigma+$ peaks $)$ within a halfbeam diameter of the stellar position is 49 per cent (i.e. not including the five cases where the peak is significantly offset from the star that they are considered to be background objects, namely HD 17093, HD 22179, HD 128167, HD 141378 and HD 150378, and also Algol, where the emission is most likely related to the radio variability). Given that all the targets in the sample are known disc hosts, and assuming the fits to the photometric points in the SEDs are accurate, it is clear that the SONS observations do not go deep enough for the non-detected disc cases. Fig. 27 shows that the number of discs detected is largely uniform as a function of spectral type (stellar luminosity) that is to be expected from such a targeted survey. Table 5 summarizes the sample numbers and detection rate by spectral type. Approximately 30 per cent of the targets that were detected at $850 \mu \mathrm{m}$ also showed flux excesses at $450 \mu \mathrm{m}$, i.e. 14 out of the original list of 100 stars showed excess flux at the $5 \sigma+$ level within a 7 arcsec radius of the stellar position. As discussed for individual targets (Section 5), the $450 \mu \mathrm{m}$ photometry has been used to constrain further the fitting of the SED and in some cases offers improved angular resolution ( $~ 7.5$ or $\sim 10$ arcsec with smoothing) for extended structures. 


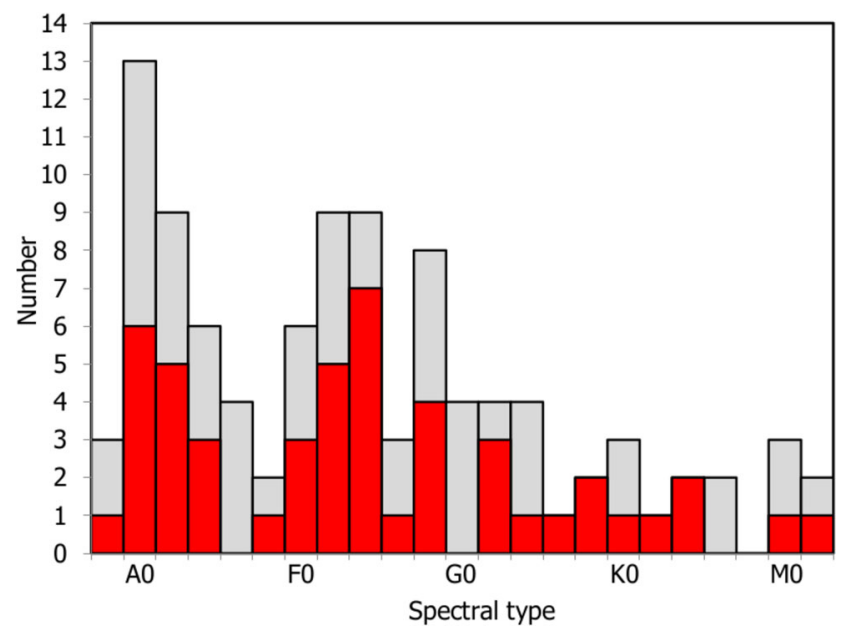

Figure 27. The number of discs detected at $850 \mu \mathrm{m}$ (red bars) as a function of host star spectral type. The grey bars represent the non-detections within the survey.

Table 5. Summary of the sample numbers and detections (at $850 \mu \mathrm{m}$ ) by spectral type. The detections do not include the extreme offset cases (HD 17093, HD 22179, HD 128167, HD 141378 and HD150378), as well as Algol (HD 19356) where the emission is likely linked to radio variability i.e. not originating from a circumstellar disc. The errors in the detection fraction are derived assuming a binomial distribution according to $d f=$ $\sqrt{f(1-f) / N}$, where $f$ is the detection fraction and $N$ the number in the sample.

\begin{tabular}{lccc}
\hline $\begin{array}{l}\text { Spectral } \\
\text { type }\end{array}$ & $\begin{array}{c}\text { Number } \\
\text { in sample }\end{array}$ & $\begin{array}{c}\text { Number } \\
\text { detected }\end{array}$ & $\begin{array}{c}\text { Detection } \\
\text { fraction (per cent) }\end{array}$ \\
\hline B & 3 & 1 & $33 \pm 4.7$ \\
A & 34 & 15 & $44 \pm 5.0$ \\
F & 35 & 20 & $57 \pm 5.0$ \\
G & 15 & 7 & $47 \pm 5.0$ \\
K & 8 & 4 & $50 \pm 5.0$ \\
M & 5 & 2 & $40 \pm 4.9$ \\
\hline
\end{tabular}

\subsection{Disc morphology and sizes}

Within the sample, 16 discs have been spatially resolved, with deconvolved measured radii ranging from 40 to 800 au (as shown in Tables 3 and 4). If the disc is composed of small grains, these will be hotter than their blackbody equivalent, and hence the disc size will be larger than that determined from the SED fit, according to equation 1 (see Section 4.3). Larger grains will behave more like blackbodies with the ratio of the measured (observed) radius $\left(R_{\mathrm{fit}}\right)$ to that derived from the SED fit $\left(R_{\mathrm{BB}}\right)$ approaching unity. Fig. 28 shows the ratio of $R_{\mathrm{fit}} / R_{\mathrm{BB}}$ plotted against stellar luminosity. It can be seen that the ratio is greater than unity in the vast majority of cases and typically less than 10 . This behaviour implies the existence of planetesimals at larger radii than those derived by assuming a simple blackbody fit to the SED.

The general trend is for the discs around lower luminosity stars to be larger than expected based on their blackbody dust grain properties. The ratio is seen to decline as $\propto L_{*}^{-0.2}$, as shown in Fig. 28. A number of papers have compared the disc sizes resolved by, for example, Herschel, relative to the expectation from blackbody emission, and found that, although the ratio was typically less than 4, there was clear evidence of a decline with increasing stellar luminosity (Booth et al. 2013; Morales et al. 2013; Pawellek et al. 2014; Pawellek \& Krivov 2015). This decline was interpreted as being

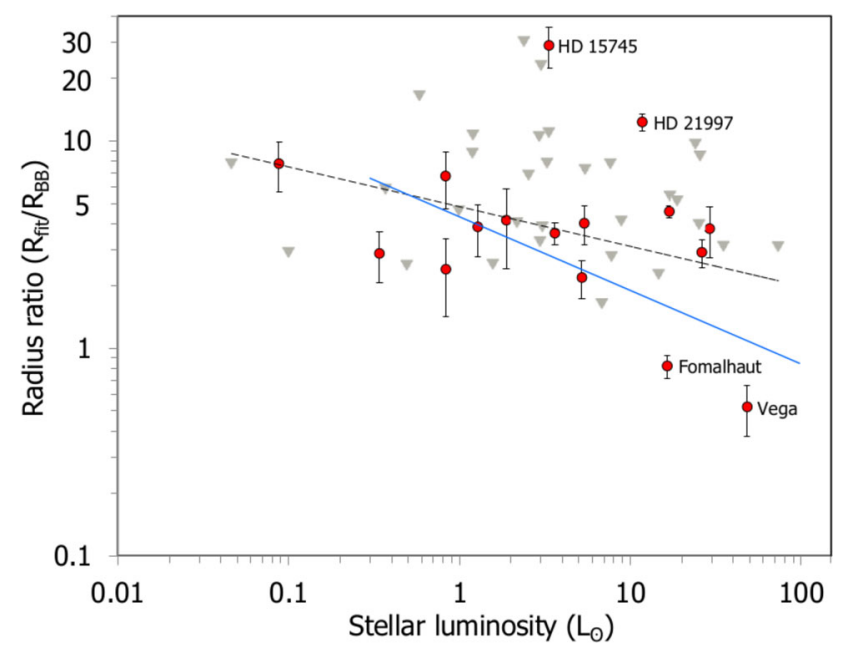

Figure 28. The ratio of the measured disc radius $\left(R_{\mathrm{fit}}\right)$ to that derived from the SED (assuming the dust grains behave as perfect blackbodies; $R_{\mathrm{BB}}$ plotted against stellar luminosity. The inverted triangles represent upper limits for the cases in which the disc is unresolved. The dashed line is a fit to the data points for which the disc is resolved (i.e. the upper limits are not included in the fit). The solid blue line is based on fitting the observed SED from Herschel observations assuming a dust composition dominated by ice and silicates (Pawellek \& Krivov 2015).

consistent with the expectation from blowout, where higher luminosity stars have removed the smallest grains via radiation pressure, leaving relatively large, blackbody-like dust grains, whilst stars of lower luminosity should have super-heated grains. For comparison, Fig. 28 also shows the ratio based on fitting the observed SED from Herschel observations, assuming a model in which the dust composition mixture is dominated by ice and silicates (Pawellek \& Krivov 2015). The SONS results support a similar behaviour, although with a larger scatter than seen in the Herschel data and a lower number of resolved sources. Some of the difference between the SCUBA-2 and Herschel results is to be expected due to the different methods used to measure the resolved disc sizes. The Herschel papers listed above all fit narrow rings to the data, whilst in this paper 2D Gaussians are adopted. In cases where the disc is wide, a Gaussian fit will tend to give a radius closer to the outer radius, whereas a narrow ring fit will give a radius close to that dominating the emission and hence closer to the equivalent blackbody radius.

There are two prominent (high) outliers in the plot with ratios above a value of 10 (namely HD 15745 and HD 21997). As discussed in Section 5.12, the disc around HD 15745 appears to be extended at $850 \mu \mathrm{m}$, but shows no such morphology at farIR wavelengths (appearing unresolved in Herschel/PACS 100 and $160 \mu \mathrm{m}$ observations). The scattered light image (Kalas et al. 2007b; Schneider et al. 2014) seems to suggest that the disc does extend to a radius close to that seen in the SCUBA-2 image ( $\sim 500 \mathrm{au})$, although the scattered light disc has a PA of $\sim 22.5^{\circ}$, compared to the $\sim 164^{\circ}$ from the SONS $850 \mu \mathrm{m}$ image. It is unclear what could cause such a discrepancy, although it has been hypothesized that it may be related to interstellar winds (Schneider et al. 2016). HD 21997 is only marginally resolved based on an extended $3 \sigma$ contour to the south (Section 5.15). Nonetheless, a previous multiwavelength analysis does suggest the possibility of a disc extending to at least 490 au (Moór et al. 2013), and it is likely that the radius ratio is so high because the disc is very wide and the SED is dominated by warmer grains at the inner edge $(\sim 50 \mathrm{au})$. 


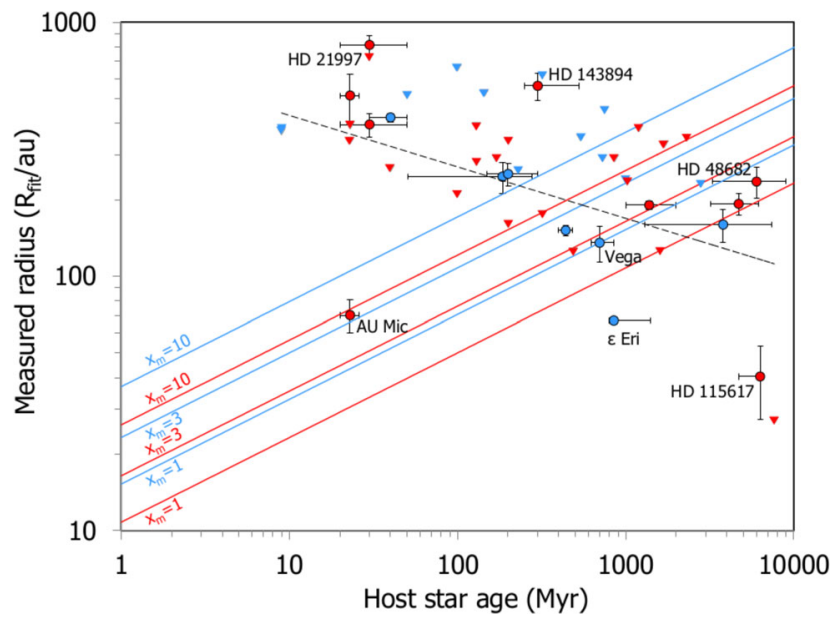

Figure 29. The measured radius as a function of host star age for the 16 targets in the SONS survey for which the disc radius was resolved, as represented by the red and blue circles (with error bars). The inverted triangles are upper limits to the radius for the cases in which the disc is unresolved. For both the circles and triangles, red symbols represent stars in the mass range of $0.3-1.5 \mathrm{M}_{\odot}$, whilst blue covers the $1.5-3.3 \mathrm{M}_{\odot}$ range. The red and blue lines represent self-stirring models computed for the host stars with masses of $1 \mathrm{M}_{\odot}$ (red) and $2 \mathrm{M}_{\odot}$ (blue) and for three different scaling factors, $x_{m}$, adapted from Moór et al. (2015). The dashed line is a fit to the resolved disc data points, and tentatively indicates a decline in disc radius as a function of stellar age $\propto t^{-0.2}$, where $t$ is the host star age.

Vega, on the other hand, has a low ratio of 0.52 based on the fitted $850 \mu \mathrm{m}$ image (or 0.28 based on the $450 \mu \mathrm{m}$ image), meaning that the observed disc is significantly smaller than that implied assuming the dust grains are radiating as pure blackbodies. As discussed in Section 5.44, one possibility is that there is undetected, low-level emission in the SONS images, extending well beyond the lowest contours of the map, resulting in an underestimate for the measured radius of the disc. It is already known that the disc radius could extend to many hundreds of au (Su et al. 2005), and so it is possible that the far-IR emission (which sets the temperature) is dominated by halo grains at a radius significantly larger than the main belt (peaking around 200-300 au). This would, however, mean that the halo properties for Vega are different from those around other stars (e.g. AU Mic) in that such grains would have to be cooler than their blackbody equivalents. This remains an unexplained issue.

In summary, the median $R_{\mathrm{fit}}$ to $R_{\mathrm{BB}}$ ratio for all the targets is 3.8 (or 3.6 if the high and low outliers are excluded). The greater than unity ratio in the vast majority of cases implies the existence of planetesimals at larger radii than those derived from a blackbody fit. There are several anomalies that still need to be explained, including both high ratio outliers such as HD 15745 and targets, such as Vega, that have a low ratio.

After the gas dissipates from a circumstellar disc, planetesimals need to be stirred to initiate a collisional cascade. Moór et al. (2015) investigate stirring in a number of massive discs (many of which are also in this survey). Fig. 29 shows a tentative decline in the measured radius of the resolved discs in the SONS sample as a function of the host star age. The red and blue lines represent 'selfstirring' models ${ }^{5}$ computed for the host stars with masses of $1 \mathrm{M}_{\odot}$ (red) and $2 \mathrm{M}_{\odot}$ (blue) and for three different scaling factors, $x_{m}$,

\footnotetext{
${ }^{5}$ To achieve 'self-stirring' requires the formation of Pluto-sized planetesimals.
}

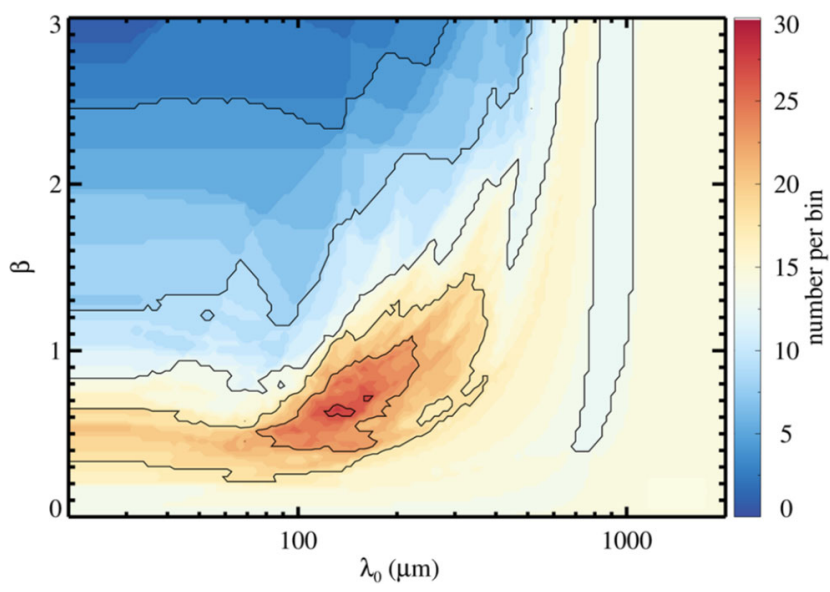

Figure 30. The $3 \sigma$ constraints of dust emissivity $(\beta)$ plotted against critical wavelength $\left(\lambda_{0}\right)$ for all the detections in the SONS survey. The contours are at levels of $5,10,15,20,25$ and 30.

adapted from Moór et al. (2015), and where $x_{m}$ is the ratio of the assumed mass of the initial protostellar disc to the minimum mass Solar nebula. The results are also in agreement with Moór et al. (2015) in that that self-stirring can explain large discs but only around old systems. For young stars with discs extending out to radii of greater than $\sim 100 \mathrm{au}$, planet stirring is currently the only explanation for the detection of dust at such large distances from the star. In this survey, such an explanation would apply to the discs detected around 49 Cet, HD 15745, HD 21997, 44 Ser, $\gamma$ Oph, HD 170773 and HR 8799.

\subsection{Spectral slopes and grain properties}

There are many instances where the absence of photometric data beyond $160 \mu \mathrm{m}$ means that the critical wavelength $\left(\lambda_{0}\right)$ and the dust emissivity index $(\beta)$ are degenerate and independent constraints are not possible. The $850 \mu \mathrm{m}$ (and $450 \mu \mathrm{m}$, when available) data greatly improve this situation for the majority of the targets in the SONS survey. Fig. 30 shows a combined contour plot of the $3 \sigma$ constraints on $\beta$ against $\lambda_{0}$ for all the detections in the survey, using the SED modelling described in Section 4.2. The plot highlights that whilst there are plenty of systems in which $\lambda_{0}$ could be quite long (and hence $\beta$ quite high), where there are constraints they tend to be around $\lambda_{0} \sim 100-300 \mu \mathrm{m}$ and $\beta \sim 0.5-1.0$. Since it is unlikely that $\lambda_{0}$ will be much greater than $300 \mu \mathrm{m}$, this assumption means that there are reasonable constraints on $\beta$ being less than 1.5. The spectral index (defined as $\alpha$, where $\alpha=2+\beta$ ) of grains in the interstellar medium typically has a value of $\alpha=4$ (i.e. $\beta=2$ ) whilst debris discs tend to have shallower spectral indices ( $\operatorname{smaller} \beta$ ), as shown by the majority of discs having $\alpha<4(\beta<2)$ in Fig. 31 . There are a few cases for which the spectral index could approach 4 or more, based on the range of $\beta$ values derived from the SED modelling. Such a steep spectrum, if confirmed, could signify a disc dominated by small grains, most likely not greater than $10 \mu \mathrm{m}$ in size (Ertel et al. 2012; Booth et al. 2013).

It is clear that there is a paucity of data between 160 and $850 \mu \mathrm{m}$ for all but the brightest discs. For example, the Herschel/SPIRE data at 250, 350 and $500 \mu \mathrm{m}$ reach the background confusion limit too quickly for fainter disc candidates to be confirmed, and so the SCUBA-2 $450 \mu \mathrm{m}$ data potentially become critical in sampling this important region of the SED for debris discs. In addition, the fact that the 450 and $850 \mu \mathrm{m}$ data are obtained simultaneously 


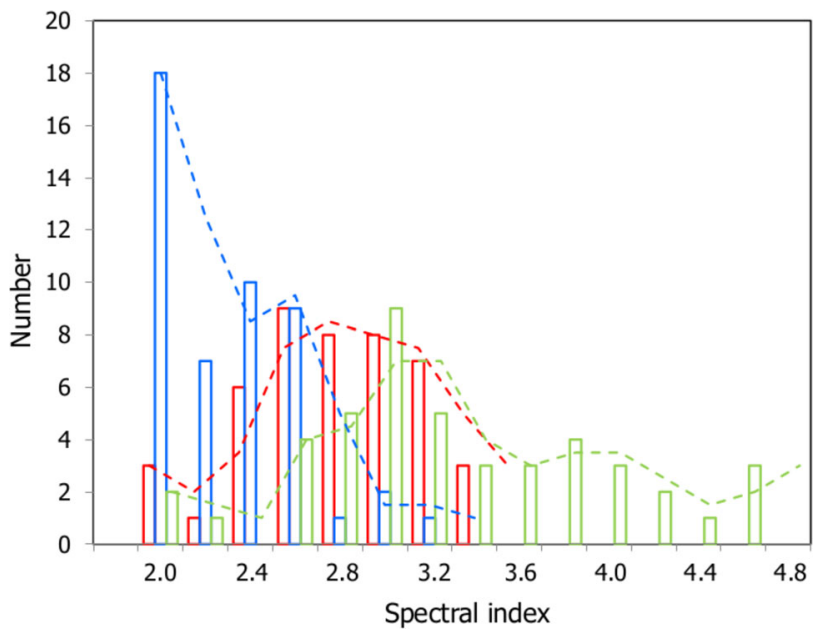

Figure 31. Histogram of the spectral indices derived from the SED modelling. The three coloured bars represent the range of $\alpha(\alpha=2+\beta)$ based on the derived $\beta$ values (blue the minimum value, red the median and green the maximum of the range).

with SCUBA-2 means that systematic biases in flux calibration should impact both wavelengths in the same way. This inherent behaviour would suggest that any high spectral indices derived directly from the ratio of 450-850 $\mu \mathrm{m}$ fluxes would appear to be genuine, but defy interpretation in terms of our understanding of the dust size distribution for discs. From the SONS survey, there is only one example in which the spectral index, derived directly from the 450:850 flux ratio, is 4 or higher, namely HD 76582. This source has a spectral index of $4.3 \pm 1.5$, with the estimated error representing a significant uncertainty in the value derived. There are also examples from Herschel observations where a steep spectrum is observed beyond $70 \mu \mathrm{m}$ (Ertel et al. 2012). Further data are needed to confirm disc signatures and to improve the $\mathrm{S} / \mathrm{N}$ ratio of (at least) some of the $450 \mu \mathrm{m}$ detections to make any definitive conclusions.

The use of the modified blackbody is a simplified way of taking into account the absorption and emission efficiencies of the grains in the disc (Backman \& Paresce 1993). The radiative efficiency of individual grains depends on a variety of different properties including the molecular composition, molecular structure, size and porosity (e.g. Draine 2006). Even if it is assumed that the composition and structure of particles are the same throughout the disc, there will be a distribution of particle sizes, and, potentially, grains at a wide variety of distances from the star. Hence, the shape of the SED for the disc as a whole will depend on the summation of the emission from particles at a range of temperatures (Backman \& Paresce 1993; Gáspár et al. 2012). Resolved images can help break this degeneracy. For example, the low $\beta$ value for AU Mic is largely due to the main belt being wide, extending from $\sim 8$ to $40 \mathrm{au}$, with a halo of small grains beyond this radius (Matthews et al. 2015; Schüppler et al. 2015). This means there are grains at a wide range of temperatures contributing to the SED fit, resulting in a shallow spectral slope that masks any size distribution effects. Conversely, if it is known that the ring is narrow then the spectral slope is likely to be largely due to the size distribution (Draine 2006). For a small sample of discs, MacGregor et al. (2016a) found values of $\beta$ between roughly 0.4 and 1.1 , and showed how these suggest size distributions with power law indices around 3.4, slightly less than the canonical Dohnanyi et al. (1969) value of 3.5, and considerably less than the values predicted by Gáspár et al. (2012) and Pan \&

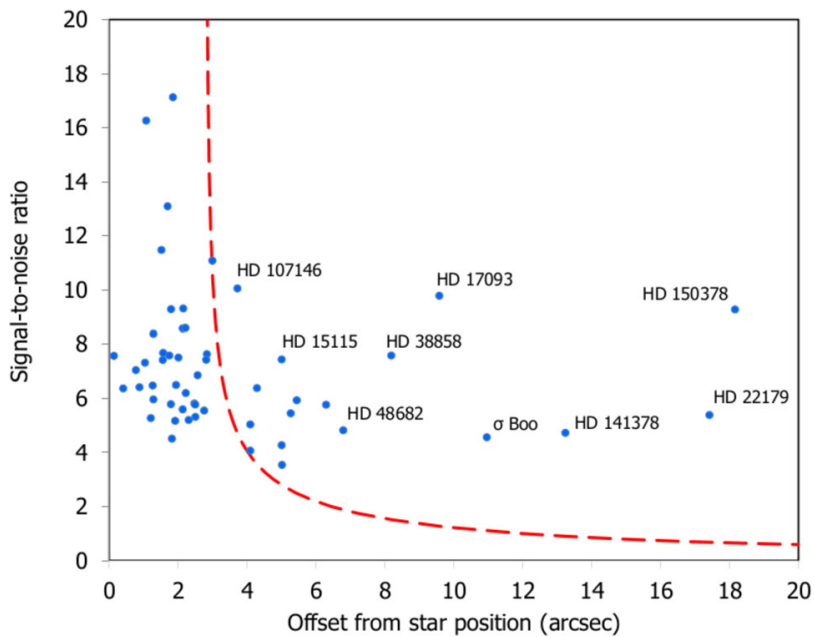

Figure 32. The offset of the flux peak from the star position as a function of $\mathrm{S} / \mathrm{N}$ at $850 \mu \mathrm{m}$ measured from the SONS survey images. The red curve represents the expected positional offset based on the statistical uncertainties and RMS telescope pointing errors of 2 arcsec in both azimuth and elevation. The peaks detected in the vicinity of HD 22179, HD 17093, $\sigma$ Boo, HD 141378 and HD 150378 are all believed to be background objects.

Schlichting (2012), taking into account more complicated physical processes. The authors propose that a wavy size distribution is a possible cause of these lower $\beta$ values. The SONS survey larger sample backs up the results of MacGregor et al. (2016a) as most discs have $\beta$ values in the $0.4-1.1$ range.

\subsection{Flux peak offsets}

As discussed in Section 5 a significant number of detections show emission offset from the star, at a distance not explainable by positional errors. The statistical uncertainty in a position is commonly expressed by $0.6 \theta(\mathrm{S} / \mathrm{N})^{-1}$ (Ivison et al. 2007), where $\theta$ is the FWHM of the telescope beam and $\mathrm{S} / \mathrm{N}$ is the peak $\mathrm{S} / \mathrm{N}$ ratio, under the assumption of Gaussian noise properties in the maps. In addition, there is also a potential error associated with the telescope pointing accuracy, which, being uncorrelated, is added in quadrature with the positional error. In the case where a target is observed over multiple nights, leading to several hours of integration time, it is possible that cumulative pointing errors may conspire to create an artificial offset between the star and the disc. No correlation, however, has been found between the magnitude of the offset and the total number of observations.

As an example, taking the RMS pointing accuracy of the telescope as 2 arcsec in azimuth and elevation, ${ }^{6}$ then at $850 \mu \mathrm{m}$ the overall positional uncertainty for a peak detected at $4 \sigma$ should be no greater than 3.5 arcsec for the effective FWHM beam size of 15 arcsec (after smoothing). Fig. 32 shows the measured offset of the flux peak from the stellar position as a function of the peak $\mathrm{S} / \mathrm{N}$ of the detection. ${ }^{7}$ Also plotted (red line) is the expected positional offset based purely on the statistical uncertainties and an RMS telescope pointing error of 2 arcsec in both azimuth and elevation. As can be

\footnotetext{
${ }^{6}$ See: http://www.eaobservatory.org/jcmt/about-jcmt/

${ }^{7}$ For HD 207129 and Fomalhaut the offset is measured from the geometric centre between the two peaks, i.e. it is assumed that the emission is distributed in a ring about the star. Likewise, for $\epsilon$ Eri the offset is measured from the geometric centre of the dust ring.
} 


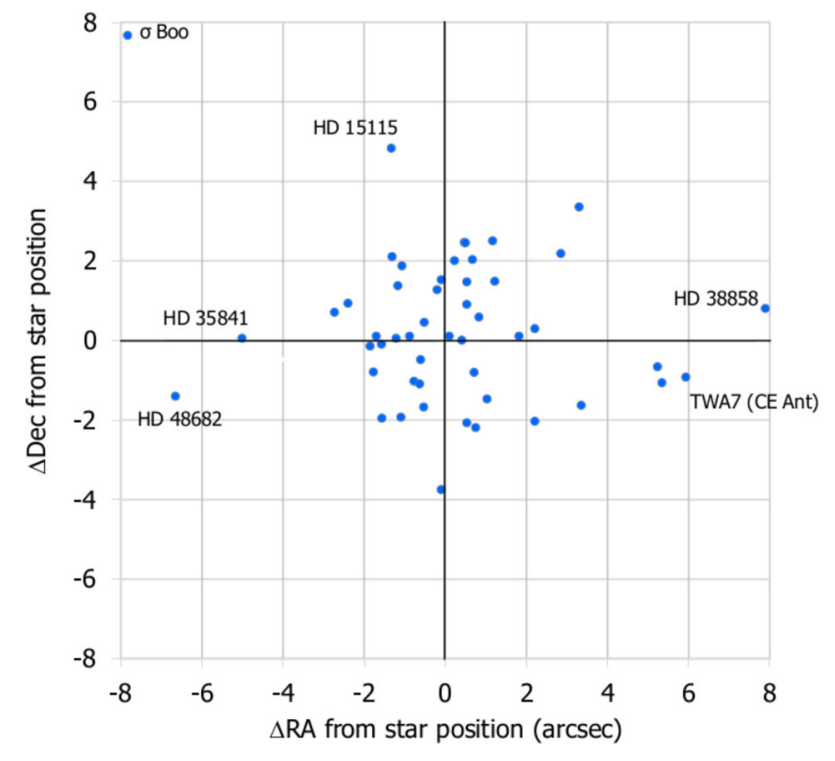

Figure 33. Correlation between the measured difference in RA and Dec. of the flux peak from the stellar position for the SONS survey sample. The names of some of the stars that show a significant offset in RA or Dec. are also given. Three of the extreme offset cases (as indicated in Fig. 32, namely HD 17093, HD 22179 and HD 150378) are not shown.

seen, the majority of the points lie to the left of the curve, suggesting that the pointing accuracy of the telescope is routinely better than 2 arcsec in both axes (which based on the pointings in this survey, taken prior to target observations, seems to be the case). In some of the more extreme cases (i.e. HD 17093, HD 22179, $\sigma$ Boo, and HD 150378 ), where the offset is $\sim 10$ arcsec or more, it is very unlikely that the detected peak is associated with the star (as discussed for individual cases in Section 5). In total, 12 out of 49 peaks fall strictly outside the expected positional error (not including the five extreme cases) based on the statistical and nominal telescope pointing uncertainties.

Why there are so many 'offset' disc cases remains a mystery. Fig. 33 shows the measured difference in RA and Dec. of the flux peak from the stellar position (ensuring that corrections are made for the proper motion of the star). It is interesting to note that there is a slight tendency for the offsets to occur predominantly in RA, i.e. in an east-west direction in the images. Periodic tracking errors from the telescope are ruled out as the the JCMT is an Alt-Az mounted telescope, and indeed, rebinning a selection of the offset case data sets in an Azimuth-Elevation coordinate frame showed no indication of any preferential positional shift. Some of these cases have speculative (i.e. unproven) explanations, usually citing the possible presence of a background source as in the case of several of the more extreme offset cases shown (see Section 6.5). The association of an offset peak with a putative disc about the star can also have a physical explanation. For instance, such emission might indicate a perturbed disc that could have detectable volatiles. Within the SONS survey sample there are a few examples of systems containing molecular $\mathrm{CO}$ gas, including the young ( $<50 \mathrm{Myr})$ A-stars HD 21997 and 49 Ceti (Zuckerman et al. 1995; Moór et al. 2011a). No CO, however, has been detected around HD 38858, the most extreme offset disc in the sample that is not believed to be a background object based on current understanding (Kennedy et al. 2015).

\subsection{Background contamination}

At the sensitivity levels achieved within the SONS survey (e.g. an average $1 \sigma$ noise level of $1.2 \mathrm{mJy} \mathrm{beam}^{-1}$ at $850 \mu \mathrm{m}$ in the central $3 \operatorname{arcmin}^{2}$ region), images will have some level of 'contamination' by high redshift galaxies (submillimetre galaxies; SMGs). Indeed, SMG occurrence is a growing issue in terms of the interpretation of debris discs as surveys go deeper. There are a number of examples in the SONS survey where asymmetric features or close-by peaks could be due to background galaxies. An example is the disc around $\mathrm{q}^{1}$ Eri (Fig. A2a) as already discussed in Section 5.5. In this case, $870 \mu \mathrm{m}$ observations with APEX/LABOCA (Liseau et al. 2010) showed evidence of the east-west extension, which was very clearly seen by SCUBA-2, and identified as an isolated point-source at shorter wavelengths by Herschel (Liseau et al. 2010).

It is possible to estimate the chances of a background galaxy lying, say, within a half-beam distance from the star of interest. Assuming an average detection threshold of $4 \mathrm{mJy}(3 \sigma)$ at $850 \mu \mathrm{m}$, the source count number is $\sim 1400 \mathrm{deg}^{-2}$ (e.g. Blain et al. 1999). This surface density corresponds to $\sim 2.1$ sources in the $3 \operatorname{arcmin}^{2}$ central area of a SONS field. Alternatively, simply counting the number of $3.5 \sigma$ peaks in the same area (avoiding the central region of radius 15 arcsec, i.e. a distance of $\sim 2$ beam radii from the star), gives an average of 2.3 peaks per field, in good agreement with the galaxy count models. Therefore, the probability of a background galaxy lying within a half-beam distance of any star is crudely estimated to be around 5 per cent. This expectation means that 2-3 fields within the SONS sample could have a background galaxy within a half-beam distance of the star. Based on the discussion outlined for individual targets in Section 5, there are potentially as many as 9 out of the 49 cases (ignoring the five extreme offsets and Algol) for which the interpretation of a disc structure could be influenced by a background object. The stars for which the observed structure (or part thereof) is believed to be a background object, supported by multiwavelength imaging, are $q^{1}$ Eri, $\epsilon$ Eri, HD 107146 and $\sigma$ Boo, whilst those that require further investigation are HD 38858, HD 92945, HD 104860, HD 127821 and HD 205674.

Finally, the SONS survey amounts to 100 fields of $\sim 10 \mathrm{arcmin}^{2}$, equating to $\sim 3 \mathrm{deg}^{2}$ of total area. Even though the use of the DAISY mode means that the noise increases almost linearly with radius outside the central $3 \operatorname{arcmin}^{2}$ region (estimated maximum $1 \sigma$ noise level of $2 \mathrm{mJy}$ beam $^{-1}$ at the edge of the field; Holland et al. 2013), this performance still represents a significant depth and area to carry out an unbiased survey count of SMGs. Such a study is planned in the future.

\subsection{Disc mass evolution}

In describing how a disc evolves the key parameters to investigate are how the fractional luminosity $(f)$ and the dust mass $\left(M_{\mathrm{d}}\right)$ vary with stellar age. Understanding how stellar ages are derived and the accuracy (and consistency) of the results is fundamental in this regard (Zuckerman \& Song 2004). Different diagnostics can give conflicting results (Moór et al. 2006) and so conclusions about disc evolution depend on stellar ages being determined in a consistent manner. The values adopted in this paper, and referenced for individual stars in Section 5, have been deliberately conservative in terms of the quoted uncertainties in stellar age. For the cases in which no age uncertainty is available, a value of \pm 25 per cent has been adopted. 


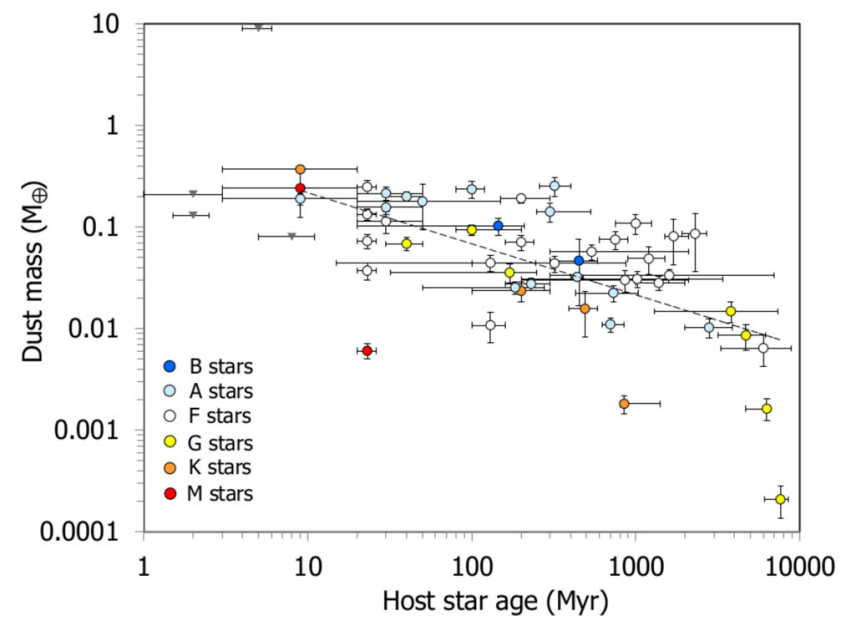

Figure 34. The derived dust mass variation with host star age for the SONS survey sample. The circular symbols represent the dust mass estimates for the debris discs in the SONS sample (all the stars have estimated ages of $>9 \mathrm{Myr}$ ). A fit to the SONS results (shown by the dashed line) suggests a decline in dust mass according to $0.8 t^{-0.5}$, where $t$ is the host star age. The inverted grey triangles represent the approximate $3 \sigma$ mass sensitivity levels from recent published surveys of circumstellar discs within clusters/associations: Upper Scorpius around $8 \pm 3 \mathrm{Myr}$ (Barenfeld et al. 2016), $\lambda$ Orionis around $5 \pm 2 \mathrm{Myr}$ (Ansdell, Williams \& Cieza 2015), Lupus $2 \pm 1 \mathrm{Myr}$ (Ansdell et al. 2016) and Chamaeleon I around $2 \pm 0.5 \mathrm{Myr}$ (Pascucci et al. 2016). Although these targets are mainly classified as premain sequence protoplanetary discs, it should be noted that some debris disc candidates are also contained in the Upper Scorpius sample.

Emission from debris discs is expected to diminish over time as the reservoir of large planetesimal bodies is depleted and the collisional time-scales increase (Wyatt 2008). Fig. 34 shows how the dust mass, calculated from the $850 \mu \mathrm{m}$ flux and the dust temperature derived from the SED fit, varies with stellar age for the SONS survey sample (circular symbols). The largest measured dust masses within the sample over the range $10-10000 \mathrm{Myr}$ are around $0.4 \mathrm{M}_{\oplus}$, whilst the smallest are around the two old G-stars, namely HD 115617 and $\tau$ Cet, with a minimum value of $2 \times 10^{-4} \mathrm{M}_{\oplus}$ ) for the latter. Although there is considerable scatter (as shown by the errors), the general trend is for a decline in dust mass as the star gets older according to $\sim t^{-0.5}$, where $t$ is the host star age. This trend is less steep than expected from a condition of steady-state collisional evolution in which $M_{\mathrm{d}} \propto t^{-1}$ (Wyatt 2008). As discussed in Panić et al. (2013), there is, however, a degree of uncertainty in this interpretation due to observational bias. As shown in Fig. 35, the younger stars in the sample tend to be more distant, meaning that the mass sensitivity for these targets is lower than for older, but closer stars (around which low-mass discs are easier to detect). This effect can certainly be seen in Fig. 34 for a few very old stars with derived masses lower than $0.01 \mathrm{M}_{\oplus}$, but not around younger stars since such stars are more distant. Hence, it is likely that at least some of the variation seen in the dust mass with stellar age plot is due to an observational bias, and not a true indication of disc mass evolution.

In more general terms, the mass in millimetre-sized grains within circumstellar discs has previously been shown to apparently decline sharply in the period around $10 \mathrm{Myr}$ (generally considered to be the 'transitional phase' between protoplanetary and debris discs; Wyatt 2008), experiencing a drop of at least two orders of magnitude to the level shown for the youngest stars (e.g. Panić et al. 2013). It is suggested that this age may represent a period of rapid accretion of

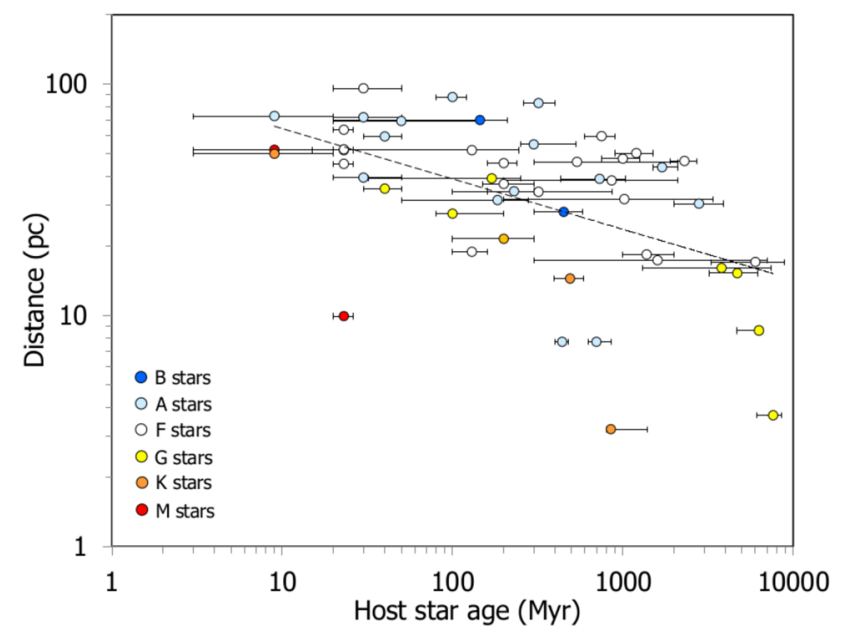

Figure 35. Distance of the star plotted against host star age for the SONS survey sample. The dashed trend line $\left(\propto t^{-0.2}\right)$ illustrates the bias of younger stars being more distant within the survey sample.

material on to planetesimals, potentially one of the final phases in the evolution of a transition disc to a debris disc (Wyatt et al. 2015).

Until the recent advent of interferometers the mass sensitivity of single-dish telescopes in the submillimetre was rather limited for protoplanetary discs since they tend to be more distant (e.g. $2 \mathrm{kpc}$ for Taurus) than the debris discs chosen in most surveys (e.g. for SONS, the criterion was a distance of $<100 \mathrm{pc}$ ). The new surveys with SMA and ALMA have shown mass sensitivities to levels as low as, for example, $\sim 0.1 \mathrm{M}_{\oplus}$ for the Upper Scorpius OB association (Barenfeld et al. 2016). Fig. 34 also shows the $3 \sigma$ mass sensitivity from a number of recent such surveys of early circumstellar disc candidates (denoted by the inverted grey triangles in the plot). The improved mass sensitivity now suggests the decline in mass is not as steep as previously suggested i.e. the concept that protoplanetary discs suddenly dissipate at $\sim 10 \mathrm{Myr}$ is no longer strongly supported by the data. Given that there are also debris discs in the recent interferometer surveys of young associations, it is likely that some such discs form at a much earlier stage than previously believed ( $<10 \mathrm{Myr}$ ), and therefore co-exist with protoplantary discs within star-forming regions. Finally, the significant fraction of the mass is expected to be contained in larger (km-sized) planetesimals, particularly for debris discs, and so the total mass of the disc may be considerably higher than the estimates presented in this paper.

Future surveys might target younger stars, allowing us to quantify how disc properties, such as the size distribution and mass, vary with age, at stages when the evolution of these properties is most rapid. At such ages, the discs may not yet have reached equilibrium following the dispersal of the protoplanetary disc, a point that would be evidenced in a difference in the properties derived for the youngest discs in this sample. For example, ALMA observations of the young disc HD 21997 have shown that whilst the dust emission is consistent with a debris disc (i.e. the fractional luminosity is too low for a protoplanetary disc), it also hosts a CO disc (Greaves et al. 2016), with well-defined Keplerian rotation that may be primordial (Kóspál et al. 2013). It is not unequivocal that systems do take on 'debris-like' dust properties before the primordial gas is dispersed, and it is clear from the most recent surveys that there is a less significant change in the bulk of the millimetre-sized grains over a short period of time around the $10 \mathrm{Myr}$ mark than previously hypothesized (Panić et al. 2013). 
Table 6. A summary of the information on the planet hosts in the SONS survey sample.

\begin{tabular}{|c|c|c|c|c|c|}
\hline $\begin{array}{l}\text { HD } \\
\text { number }\end{array}$ & $\begin{array}{l}\text { Other } \\
\text { names }\end{array}$ & Planet name & $\begin{array}{l}\text { Orbit semimajor } \\
\text { axis (au) }\end{array}$ & Eccentricity & $\begin{array}{c}M_{\text {sini }} \\
\left(M_{\text {Jup }}\right)\end{array}$ \\
\hline 10647 & $\mathrm{q}^{1}$ Eri & HD 10647 b & $2.022 \pm 0.082$ & $0.16 \pm 0.20$ & $0.93 \pm 0.24$ \\
\hline \multirow[t]{5}{*}{10700} & $\tau$ Cet & $\tau$ Cet b & $0.105 \pm 0.005$ & $0.16 \pm 0.22$ & $0.006 \pm 0.002$ \\
\hline & & $\tau$ Cet $\mathrm{c}$ & $0.195 \pm 0.011$ & $0.03 \pm 0.28$ & $0.010 \pm 0.005$ \\
\hline & & $\tau$ Cet d & $0.374 \pm 0.02$ & $0.08 \pm 0.26$ & $0.011 \pm 0.005$ \\
\hline & & $\tau$ Cet e & $0.552 \pm 0.03$ & $0.05 \pm 0.22$ & $0.014 \pm 0.007$ \\
\hline & & $\tau$ Cet $\mathrm{f}$ & $1.35 \pm 0.09$ & $0.03 \pm 0.26$ & $0.021 \pm 0.011$ \\
\hline 22049 & $\epsilon$ Eri & $\epsilon$ Eri b & $3.38 \pm 0.32$ & $0.25 \pm 0.23$ & $1.05 \pm 0.19$ \\
\hline 38858 & & HD 38858 b & $1.038 \pm 0.019$ & $0.27 \pm 0.17$ & $0.096 \pm 0.012$ \\
\hline \multirow[t]{2}{*}{82943} & & HD 82943 c & $0.742 \pm 0.013$ & $0.43 \pm 0.03$ & $1.590 \pm 0.103$ \\
\hline & & HD 82943 b & $1.185 \pm 0.022$ & $0.20 \pm 0.07$ & $1.589 \pm 0.097$ \\
\hline 113337 & & HD 113337 b & $1.033 \pm 0.035$ & $0.46 \pm 0.04$ & $2.83 \pm 0.24$ \\
\hline \multirow[t]{6}{*}{115617} & $61 \mathrm{Vir}$ & 61 Vir b & $0.050 \pm 0.001$ & $0.12 \pm 0.11$ & $0.016 \pm 0.002$ \\
\hline & & 61 Vir c & $0.217 \pm 0.004$ & $0.14 \pm 0.06$ & $0.033 \pm 0.004$ \\
\hline & & 61 Vir d & $0.475 \pm 0.008$ & $0.35 \pm 0.09$ & $0.072 \pm 0.009$ \\
\hline & GJ 581 & GJ581 e & $0.028 \pm 0.001$ & $0.03 \pm 0.01$ & $0.006 \pm 0.001$ \\
\hline & & GJ581 b & $0.041 \pm 0.001$ & $0.03 \pm 0.01$ & $0.050 \pm 0.002$ \\
\hline & & GJ581 c & $0.073 \pm 0.002$ & $0.07 \pm 0.06$ & $0.017 \pm 0.001$ \\
\hline 216956 & Fomalhaut & Fomalhaut b & 115 & $0.12 \pm 0.01$ & $<0.5^{a}$ \\
\hline \multirow[t]{4}{*}{218396} & HR 8799 & HR 8799 e & $14.5 \pm 0.5$ & 0 & $7 \pm 3^{a}$ \\
\hline & & HR 8799 d & $24 \pm 0$ & 0.1 & $7 \pm 3^{a}$ \\
\hline & & HR 8799 c & $38 \pm 0$ & 0 & $7 \pm 3^{a}$ \\
\hline & & HR 8799 b & $68 \pm 0$ & 0 & $5 \pm 2^{a}$ \\
\hline
\end{tabular}

${ }^{a}$ The mass is not $M_{\text {sini }}$ in this case, but model estimates based on their photometry.

\subsection{Planet hosts}

As outlined in Section 2, the targets in the SONS survey are selected on detectability at $850 \mu \mathrm{m}$ and distance, and hence do not represent a comprehensive exoplanet sample (i.e. it is a reasonably unbiased sample with respect to known disc-planet occurrences). There are, however, 10 known planet hosts in the sample (as summarized in Table 6 and including the $\tau$ Ceti planetary system and planet ' $b$ ' around $\epsilon$ Eridani, which remain controversial) with seven having a detected debris disc based on the observations carried out in this survey. A Fisher exact probability test (Fisher 1922), carried out on the planet/disc data within the survey, suggests the likelihood of the planet-hosting stars having a higher disc frequency than the rest of the sample is only 7.7 per cent. Three other planet hosts were not detected within the SONS survey, HD 82943 (Kennedy et al. 2013), GJ 581 (Lestrade et al. 2012) and HD 113337 (Su et al. 2013), all of which have known debris discs from Herschel observations, but were too faint to be detected at $850 \mu \mathrm{m}$ based on the current observations. The majority of stars with known planets and a debris disc are naturally chosen to be close to Sun, as shown in Fig. 36 for both SONS and recent Herschel surveys (Marshall et al. 2014a; Moro-Martín et al. 2015). It is also the case that the few directly imaged exoplanets (i.e. HD 216956 and HR 8799) are also found in debris systems. Indeed, with the exception of these systems, the planets discovered tend to be on orbits of just a few astronomical units and so will have little influence on discs with size scales of 50 au or more (see Table 6).

Other work has derived detection rates for disc systems around planet-hosting stars of 20-30 per cent, based on more targeted surveys of planet hosting stars using Spitzer and Herschel surveys (Trilling et al. 2008; Eiroa et al. 2013; Marshall et al. 2014a). Further studies identified possible trends between low-mass planets and the presence of cool dust (Wyatt et al. 2012). Since debris discs and planets form and evolve together, the amount of solids

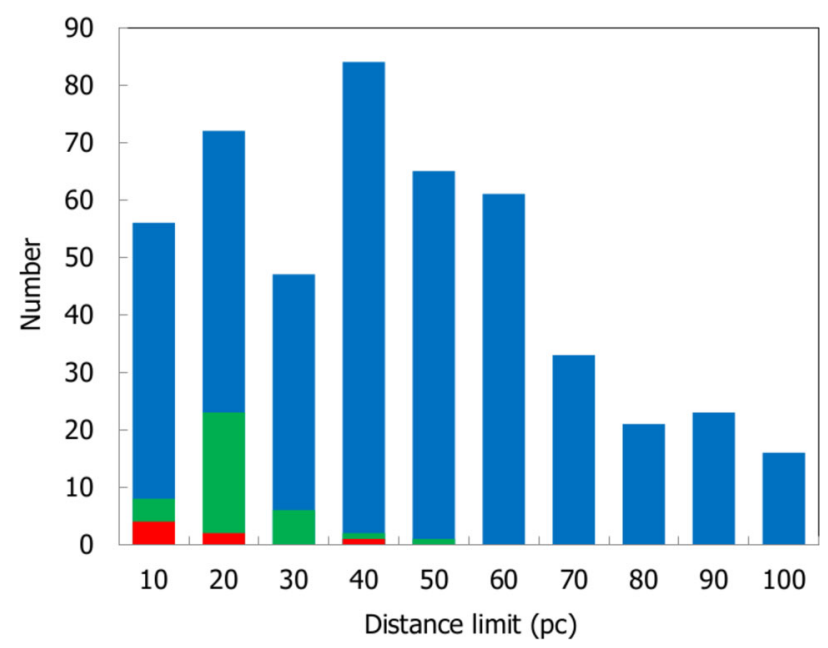

Figure 36. Histogram of the known planet hosts (blue bars) in distance bins of $10 \mathrm{pc}$ from Sun (Han et al. 2014) (i.e. the distance bin labelled ' 10 ' contains stars up to $10 \mathrm{pc}$ distance from Sun). The green and red bars represent the stars that have both known planets and detected debris discs based on recent Herschel surveys (Marshall et al. 2014a; Moro-Martín et al. 2015) and the SONS survey, respectively.

present largely determines the outcome of each, implying that discs around planet hosting stars will be brighter than those around stars with no planets (Wyatt, Clarke \& Greaves 2007). Fig. 37 shows the measured $850 \mu \mathrm{m}$ flux density for all the detected SONS targets as a function of stellar distance. Based on the small number of planet hosts in this sample, there is no obvious evidence to support this hypothesis. A more recent study of 204 FGK stars also did not find any compelling evidence that debris discs are more common (or, indeed, more dusty) around stars harbouring planets 


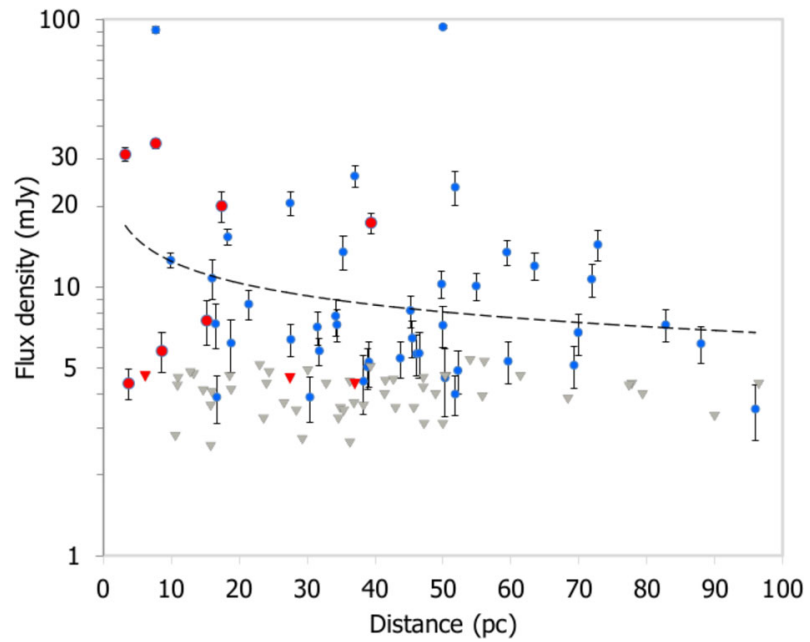

Figure 37. The measured $850 \mu \mathrm{m}$ flux density against distance for the detected discs in the SONS survey. The blue circles represent disc detections, whilst the inverted triangles are the $3 \sigma$ upper flux limits. The red filled circles and triangles indicate the known planet hosts in the sample. The dashed curve is a fit to the detected discs only as a function of distance.

(Moro-Martín et al. 2015). In the current era of planet hunting, dynamical studies of debris discs can provide a unique avenue for the detection of sub-Jupiter mass planets at large orbital radii (e.g. see fig. 11 in Wyatt 2008).

\section{CONCLUSIONS}

The SONS survey has detected $850 \mu \mathrm{m}$ emission from the vicinity of 55 stars in a sample of 100 targets. At least five of these detections are believed to be background sources, and one is thought to be due to radio emission variability rather than emission from a disc. The remainder trace dust grains up to a few millimetres in size and, by inference, the distribution of planetesimals around the host stars. The legacy of the survey is a catalogue of fluxes and images, and better characterization of the detected discs via modelling of their SEDs to derive dust temperatures and masses. Key results from the SONS survey include:

(1) The number of detected discs at $850 \mu \mathrm{m}$ from single-dish telescope observations has doubled from 24 known pre-SONS (mainly from JCMT, CSO and APEX) to 49;

(2) Approximately one-third (16 out of 49 detected discs) have been spatially resolved by the $850 \mu \mathrm{m}$ beam of the JCMT, allowing a measurement of the disc size;

(3) From the observed radial profiles, the discs are around (110) $\times$ the size of the Edgeworth-Kuiper belt in our own Solar system (assumed diameter of $100 \mathrm{au}$ );

(4) The majority of the measured $850 \mu \mathrm{m}$ disc radii are substantially larger than expected based on the blackbody fit to the SED, implying the existence of planetesimals at large radii from the host stars;

(5) The ratio of the observed (measured) disc radius to that derived from the blackbody fit also declines with stellar luminosity according to $L_{*}^{-0.2}$. This trend is consistent with the higher luminosity stars having removed the smallest grains, leaving behind relatively large (blackbody-like) dust grains;

(6) The dust spectral index $(\alpha)$ is constrained to be between values of 2.5 and 3.5 for the vast majority of the discs in the SONS survey sample, less than the typical value of 4 found in the interstellar medium;

(7) As surveys go deeper in terms of sensitivity, interpretation of disc structures becomes more difficult due to the inevitable contamination by background (notably high redshift) galaxies. There are a number of examples within SONS for which the presence of a background galaxy could help to explain the observed disc morphology;

(8) The fractional dust luminosity covers the wide range of $10^{-1}$ $10^{-5}$ compared to a value of $\sim 10^{-7}$ for the Edgeworth-Kuiper belt. All of the discs in the survey have $f$ values of less than the $10^{-2}$ threshold usually adopted for debris discs, with the exception of HD $98800(f \sim 0.1)$ and is usually classified as a protoplanetary rather than a debris disc;

(9) The most massive discs are around $0.5 \mathrm{M}_{\oplus}$ whilst the least massive are in the $10^{-2} \mathrm{M}_{\oplus}$ range (with one exception, a tiny $2 \times$ $10^{-4} \mathrm{M}_{\oplus}$ ). The least massive range of $10^{-2} \mathrm{M}_{\oplus}$ is a similar level to the Edgeworth-Kuiper belt;

(10) The amount of dust appears to decline roughly as $t^{-0.5}$, where $t$ is the host star age, consistent with theories of steady state collisional evolution models (with the caveat that at least some of this variation may be due to an observational bias); and

(11) Although the SONS survey does not represent a planet-host sample, a disc detection around many of the nearest stars means that such systems will become key planet-search targets for the future.

Just as diverse outcomes of planetary system architectures are now recognized (e.g. via Kepler; Batalha 2014), so have recent debris disc surveys (including SONS) revealed a large diversity in disc geometries. The observed diversity in exo-planetary system architectures implies that the debris material should be distributed in different ways. Establishing how varied debris discs are is thus a key piece of information that will help link the formation and evolution of planetary systems with the evolution of planetary building blocks (planetesimals). Building such empirical bridges between discs and planets brings us closer to unravelling the physical processes that led to the formation of planetary systems. Surveys such as SONS, via the study of the location, mass, morphology, and dust properties of these discs, highlight the submillimetre as an important waveband to address these questions. One of the key legacy goals of the SONS survey was indeed to lay the foundation for future, high angular resolution studies with existing and new facilities such as SMA, ALMA and JWST, with a view, for example, to studying belts of planetesimals in fine detail.

\section{ACKNOWLEDGEMENTS}

During the period of these observations the James Clerk Maxwell Telescope was operated by the Joint Astronomy Centre on behalf of the Science and Technology Facilities Council of the United Kingdom, the National Research Council of Canada and the Netherlands Organisation for Pure Research. Additional funds for the construction of SCUBA-2 were provided by the Canada Foundation for Innovation. MCW acknowledges the support of the European Union through ERC grant number 279973. GMK is supported by the Royal Society as a Royal Society University Research Fellow. MB acknowledges support from a FONDECYT Postdoctoral Fellowship, project no. 3140479 and the Deutsche Forschungsgemeinschaft through project $\mathrm{Kr} 2164 / 15-1$. JPM is supported by a UNSW Vice Chancellor's postdoctoral research fellowship. The work of OP is supported by the Royal Society through a Royal Society Dorothy Hodgkin fellowship. GJW gratefully acknowledges support from 
the Leverhulme Trust. This research has made use of the Exoplanet Orbit Database, the Exoplanet Data Explorer at exoplanets.org and the SIMBAD data base operated at CDS, Strasbourg, France. The Starlink software (Currie et al. 2014) is currently supported by the East Asian Observatory. This research used the facilities of the Canadian Astronomy Data Centre operated by the National Research Council of Canada with the support of the Canadian Space Agency.

\section{REFERENCES}

Acke B. et al., 2010, A\&A, 540, A125

Andrews S., Czekala I., Wilner D. J., Espaillat C., Dullemond C. P., Hughes A. P., 2010, ApJ, 710, 462

Ansdell M., Williams J. P., Cieza L. A., 2015, ApJ, 806, 221 A

Ansdell M. et al., 2016, ApJ, 828, 46

Ardila D. R. et al., 2004, ApJ, 617, L147

Aumann H. H. et al., 1984, ApJ, 278, L23

Backman D., Paresce F., 1993, in Levy E. H., Lunine J. I., eds, Protostar and Planets III, Univ. Arizona Press, Tucson, p. 1253

Backman D. et al., 2016, ApJ, 690, 1522

Barenfeld S. A., Carpenter J. M., Ricci L., Isella A., 2016, ApJ, 827, 142

Barrado y Navascués D., 2006, A\&A, 459, 511B

Barrado y Navascués D., Stauffer J. R., Jayawardhana R., 2004, ApJ, 614, 386

Barry D. C., 1988, ApJ, 334, 436

Batalha N., 2014, PNAS, 111, 35, 12647

Beichman C. et al., 2006, ApJ, 652, 1674

Benedict G. F. et al., 2006, AJ, 132, 2206

Béthermin M. et al., 2012, A\&A, 542, 58

Bintley D. et al., 2014, in Holland W. S., Zmuidzinas J., eds, Proc SPIE Conf. Ser. Vol. 9153, Millimeter, Submillimeter and Far-Infrared Detectors and Instrumentation for Astronomy VII, SPIE, Bellingham, p. 3

Blain A. W., Smail I., Ivison R. J., Kneib J.-P., 1999, ApJ, 512, L87

Boley A. C., Payne M. J., Corder S., Dent W. R. F., Ford E. B., Shabram M., 2012, ApJ, 750, L21

Bonfanti A., Ortolani S., Piotto G., Nascimbeni V., 2012, A\&A, 575, A18

Bonsor A., Kennedy G. M., Crepp J. R., Johnson J. A., Wyatt M. C., Sibthorpe B., Su K. Y. L., 2013, MNRAS, 431, 3025

Booth M., Wyatt M. C., Morbidelli A., Moro-Martín A., Levison H. F., 2009, MNRAS, 399, 385

Booth M. et al., 2013, MNRAS, 428, 1263

Booth M. et al., 2016, MNRAS, 460, L10

Booth M. et al., 2017, MNRAS, 469, 3200

Bryden G. et al., 2009, ApJ, 705, 1226

Burns J. A., Lamy P. L., Soter S., 1979, Icarus, 40, 1

Butler R. P. et al., 2006, ApJ, 646, 505

Carpenter J., Wolf S., Schreyer K., Launhardt R., Henning T., 2005, AJ, $129,1049 \mathrm{C}$

Carpenter J. et al., 2009, ApJS, 181, 197

Chapin E. L. et al., 2013, MNRAS, 430, 2545

Chavez-Dagostino M. et al., 2016, MNRAS, 462, 2285

Chen C. H. et al., 2005, ApJ, 634, 1372

Chen C. H., Fitzgerald M. P., Smith P. F., 2008, ApJ, 689, 589

Chen C. H. et al., 2014, ApJS, 211, 25C

Choquet E. et al., 2016, ApJ, 817, 2C

Choquet E. et al., 2017, ApJ, 834, 12C

Chrysostomou A., 2010, Highlights Astron., 15, 797

Churcher L., Wyatt M. C., Smith R., 2011, MNRAS, 410, 2

Cieza L. A. et al., 2013, ApJ, 762, 100

Corder S. et al., 2009, ApJ, 660, L65

Cultri R. M. et al., 2003, 2MASS All Sky Catalog of Point Sources, NASA/IPAC Infrared Science Archive

Currie M. J., Berry D. S., 2013, Starlink User Note 95, KAPPA Kernel Application Package, Joint Astronomy Centre, Hilo, Hawaii

Currie M. J., Berry D. S., Jenness T., Gibb A. G., Bell G. S., Draper P. W., 2014, in Manset N., Forshay P., eds, ASP Conf. Ser, Vol. 485,
Astronomical Data Analysis Software and Systems XXIII, Astron. Soc. Pac., San Francisco, p. 391

David T. J., Hillenbrand L. A., 2015, ApJ, 804, 146

Dempsey J. T. et al., 2013, MNRAS, 430, 2534

Dent W. R. F., Walker H. J., Holland W. S., Greaves J. S., 2000, MNRAS, 314,702

Dent W. R. F. et al., 2013, PASP, 125, 477

Dent W. R. F. et al., 2014, Science, 343, 1490

Deschamps R., Braun K., Jorissen A., Siess L., Baes M., Camps P., 2015, A\&A, 577, A55

Desidera S. et al., 2011, A\&A, 529, 54

Di Folco E., Thévenin F., Kervella P., Domiciano de Souza A., Coudé du Foresto V., Ségransan D., Morel P., 2004, A\&A, 426, 601

Dohnanyi S., 1969, JGR, 74, 2531

Doyon R., Lafrenière D., Artigau E., Malo L., Marois C., 2010, in Boccaletti A., ed., Proc. Conf. In the Spirit of Lyoy 2010: Direct Detection of Exoplanets and Circumstellar Disks, Univ. Paris Diderot, Paris, p. 42

Draine B. T., 2006, ApJ, 636, 1114D

Duchêne G. et al., 2014, ApJ, 784, 148

Ducourant C., Teixeira R., Galli P. A. B., Le Campion J. F., Krone-Martins A., Zuckerman B., Chauvin G., Song I., 2014, A\&A, 563, A121

Dulk G. A., 1985, AR\&A, 23, 169

Eiroa C. et al., 2013, A\&A, 555, A11

Ertel S. et al., 2012, A\&A, 541, 148

Farihi J., 2016, A\&A, 541, 148

Fisher R. A., 1922, J. R. Stat. Soc., 85, 87

Fujiwara H. et al., 2012, ApJ, 759, 18

Fujiwara H. et al., 2013, A\&A, 550, A45

Galicher R., Marois C., Zuckerman B., Macintosh B., 2013, ApJ, 769, 42

Gáspár A., Psaltis D., Rieke G. H., Ozel F., 2012, ApJ, 754, 74G

Gibb A. G., Jenness T., Economu F., 2013, Starlink User Note 265, PICARD - A Pipeline for Combining and Analysing Reduced Data, Joint Astronomy Centre, Hilo, Hawaii

Gillet F. C., 1986, in Israel F., ed., Light on Dark Matter: Proceedings of the First Infra-Red Astronomical Satellite Conference, D. Reidel Publishing Co., p. 61

Golimowski D. A. et al., 2011, AJ, 142, 30

Greaves J. S. et al., 1998, ApJ, 506, L133

Greaves J. S., Wyatt M. C., Holland W. S., Dent W. R. F., 2004, MNRAS, 351, L54

Greaves J. S. et al., 2005, ApJ, 619, L187

Greaves J. S. et al., 2016, ApJ, 791, L11

Greaves J. S. et al., 2016, MNRAS, 461, 3910

Habing S. et al., 2001, A\&A, 365, 545

Han E. et al., 2013, PASP, 126, 827

Hatzes A. P. et al., 2000, ApJ, 544, L145

Heap S., Lindler D. J., Lanz T. M., Cornett R. H., Hubeny I., Maran S. P., Woodgate B., 2000, ApJ, 539, 435

Hillenbrand L. A. et al., 2008, ApJ, 677, 630

Hines D. C. et al., 2007, ApJ, 671, 165

Holland W. S. et al., 1998, Nature, 392, 788

Holland W. S. et al., 1999, MNRAS, 303, 659

Holland W. S. et al., 2003, ApJ, 582, 1141

Holland W. S. et al., 2013, MNRAS, 430, 2513

Holmberg J., Nordström B., Anderson J., 2007, A\&A, 475, 519

Holmberg J., Nordström B., Anderson J., 2009, A\&A, 501, 941

Howard A. W. J., Fulton B. J., 2016, PASP, 128, 4401

Hughes A. M., Wilner D. J., Andrews S. M., Williams J. P., Su K. Y. L., Murray-Clay R. A., Qi C., 2011, ApJ, 740, 38

Hughes A. M. et al., 2012, ApJ, 750, 82H

Hughes A. M. et al., 2017, ApJ, 839, 86

Ishihara D. et al., 2010, A\&A, 514, 1I

Ivison R. J. et al., 2007, MNRAS, 380, 199

Jayawardhana R., Fisher S., Hartmann L., Telesco C., Piña R., Fazio G., 1998, ApJ, 503, L79

Jenness T., Ecomomou F., 2015, Astron. Comput., 9, 40

Jenness T. et al., 2015, Astron. Comput., 12, 146

Jones J. et al., 2015, ApJ, 813, 58J 
Jourdain de Muizon M. et al., 1999, A\&A, 350, 875

Kalas P., Graham J. R., Clampin M. C., 2005, Nature, 435, 1067

Kalas P., Duchene G., Fitzgerald M., Haghighipour P., Graham J. R., 2007, ApJ, 671, L161

Kalas P., Fitzgerald M. P., Graham J. R., 2007, ApJ, 661, L85

Kalas P. et al., 2008, Sci, 322, 1345

Kalas P., Graham J. R., Fitzgerald M. P., Clampin M., 2013, ApJ, 775, 56

Kennedy G. M., Wyatt M. C., 2011, MNRAS, 412, 2137

Kennedy G. M., Wyatt M. C., 2014, MNRAS, 444, 3164

Kennedy G. M., Wyatt M. C., Sibthorpe B., Phillips N. M., Matthews B. C., Greaves J. S., 2012, MNRAS, 426, 2115

Kennedy G. M., Wyatt M. C., Bryden G., Wittenmyer R., Sibthorpe B., 2013, MNRAS, 436, 898

Kennedy G. M. et al., 2015, MNRAS, 449, 3121

Koerner D. W., Ressler M. E., Werner M. W., Backman D. E., 1998, ApJ, 503, L83

Koerner D. W., Jensen E. L. N., Cruz K. L., Guild T. B., Gultekin K., 2000, ApJ, 533, L37

Koerner D. W., Sargent A. I., Ostroff N. A., 2001, ApJ, 560, L181

Koerner D. W. et al., 2010, ApJ, 710, 26

Kóspál Á., Ardila D. R., Moóor A., ÁAbrahám P., 2009, ApJ, 700, L73

Kóspál Á. et al., 2013, ApJ, 776, 77

Krist J. E., Ardila D. R., Golimowski D. A., 2005, AJ, 129, 1008

Krist J. E. et al., 2010, ApJ, 140, 1051

Krist J. E. Stapelfeldt K. R., Bryden G., Plavchan P., 2012, AJ, 144, 45

Krivov A. V., 2015, RAA, 10, 383

Lagrange A. M., Backman D. E., Artymowicz P., 2000, in Mannings V., Boss A. P., Russell S. S., eds, Protostar and Planets IV, Univ. Arizona Press, Tucson, p. 639

Lawler S. M. et al., 2014, MNRAS, 444, 2665

Lebouteiller V., Barry D. J., Spoon H. W. W., Bernard-Salas J., Sloan G. C., Houck J. R., Weedman D. W., 2011, ApJS, 196, 8

Lestrade J.-F., Thilliez E., 2015, A\&A, 576, A72

Lestrade J.-F., Mutel R. L., Preston R. A., Phillips R. B., 1988, ApJ, 328, 232

Lestrade J.-F. et al., 2012, A\&A, 548, A86

Liseau R. et al., 2008, A\&A, 480, L47

Liseau R. et al., 2010, A\&A, 518, L132

Liu M. C., Matthews B. C., Williams J. P., Kalas P. G., 2004, ApJ, 608, 526

Löhne T. et al., 2012, A\&A, 537, A110

Low F., Smith P. S., Werner M., Chen C., Krause V., Jura M., Hines D. C., 2005, ApJ, 631, 1170

MacGregor M. A. et al., 2013, ApJ, 762, L21

MacGregor M. A., Wilner D. J., Andrews S. M., Lestrade J.-F., Maddison S., 2015, ApJ, 811, 47

MacGregor M. A. et al., 2016a, ApJ, 823, 79M

MacGregor M. A., Lawler S. M., Wilner D. J., Matthews B. C., Kennedy G. M., Booth M., Di Francesco J., 2016b, ApJ, 828, 113M

MacGregor M. A. et al., 2017, ApJ, 842, 8

Mamajek E. E., Bell C. P. M., 2014, MNRAS, 445, 2169

Mamajek E. E., Hillenbrand L. A., 2008, ApJ, 687, 1264

Marino S. et al., 2016, MNRAS, 460, 2933

Marino S. et al., 2017a, MNRAS, 465, 2595

Marino S., Wyatt M. C., Kennedy G. M., Holland W. S., Matrà L., Shannon A., Ivison R. J., 2017b, MNRAS, 469, 3518

Markwardt C. B., 2009, in Bohlender D. A., Durand D., Dowler P., eds, ASP Conf. Ser. Vol. 411, Astronomical Data Analysis and Systems XVIII, Astron. Soc. Pac., San Francisco, p. 251

Marois C., Macintosh B., Barman T., Zuckerman B., Song I., Patience J., Lafrenière D., Doyon R., 2008, Science, 322, 1348

Marois C., Zuckerman B., Konopacky Q. M., Macintosh B., Barman T., 2010, Nature, 468, 1080

Marsh K. A., Velusamy T., Dowell C. D., Grogan K., Beichman C. A., 2005, ApJ, 620, L47

Marsh K. A., Dowell C. D., Velusamy T., Grogan K., Beichman C. A., 2006, ApJ, 646, L77

Marshall J. P. et al., 2011, A\&A, 529, A117
Marshall J. P. et al., 2014a, A\&A, 565, 15N

Marshall J. P. et al., 2014b, A\&A, 570, A114

Marshall J. P., Booth M., Holland W., Matthews B. C., Greaves J. S., Zuckerman B., 2016, MNRAS, 459, 2893

Matrà L. et al., 2017, ApJ, 842, 9

Matthews B. C. et al., 2007a, PASP, 119, 842

Matthews B. C., Kalas P. G., Wyatt M. C., 2007b, ApJ, 663, 1103

Matthews B. C., Krivov A. V., Wyatt M. C., Bryden G., Eiroa C., 2014, in Beuther H., Klessen R. J., Dullemond C. P., Henning T., eds, Protostar and Planets VI, Univ. Arizona Press, Tucson, p. 521

Matthews B. C. et al., 2015, ApJ, 811, 100

Melis C., Zuckerman B., Rhee J. H., Song I., 2010, ApJ, 717, L57

Miller B. A., Budaj J., Richards M., KoubskÝ P., Peters G. J., 2006, ApJ, 656,1075

Milli J. et al., 2017, A\&A, 597, L2

Monnier J. D. et al., 2012, ApJ, 761, L3

Montesinos B., Eiroa C., Mora A., Merín B., 2009, A\&A, 495, 901

Montesinos B. et al., 2016, A\&A, 593, A51

Moòr A., Abrahm P., Derekas A., Kiss C., Kiss L. L., Apai D., Grady C., Henning T., 2006, ApJ, 644, 525

Moòr A. et al., 2011a, ApJ, 740, L7

Moòr A. et al., 2011b, ApJS, 193, 4

Moòr A. et al., 2013, ApJ, 777, L25

Moòr A. et al., 2015, MNRAS, 447, 577

Morales F., Rieke G. H., Werner M. W., Bryden G., Stapelfeldt K. R., Su K. Y. L., 2011, ApJ, 730, 29

Morales F., Bryden G., Werner M. W., Stapelfeldt K. R., 2013, ApJ, 776, 111

Morales F., Bryden G., Werner M. W., Stapelfeldt K. R., 2016, ApJ, 831, 97

Moro-Martín A. et al., 2015, ApJ, 801, 143

Moshir M. et al., 1990, IRAS Faint Source Catalogue, version 2. Jet Propulsion Laboratory, Pasadena

Mouillet D., Lagrange A.-M., Beuzit J.-L., Renaud N., 1997, A\&A, 324, 1083

Najita J., Williams J. P., 2005, ApJ, 635, 625

Nilsson R., Liseau R., Brandeker A., Olofsson G., Risacher C., Fridlund M., Pilbratt G., 2009, A\&A, 508, 1057

Nilsson R. et al., 2010, A\&A, 518, A40

Olofsson J. et al., 2016, A\&A, 591, 108

Pace G., 2015, A\&A, 551, L8

Pagano M., Truitt A., Young P. A., Shim S.-H., 2015, ApJ, 803, 90

Pan M., Schlichting H. E., 2012, ApJ, 747, 113P

Pan M., Nesvold E. R., Kuchner M. J., 2016, ApJ, 832, 81P

Panić O. et al., 2013, MNRAS, 435, 1037

Pascucci I. et al., 2016, ApJ, 831, 128

Pawellek N., Krivov A. V., 2015, MNRAS, 454, 3207P

Pawellek N. et al., 2014, ApJ, 792, 65

Perryman M. A. C. et al., 1997, A\&A, 323, L49

Phillips N., 2011, PhD thesis, Univ. Edinburgh

Plavchan P., Werner M. W., Chen C. H., Stapelfeldt K. R., Su K. Y. L., Stauffer J. R., Song I., 2009, ApJ, 698, 1068

Pollack J. B., Hollenbach D., Beckwith S., Simonelli D. P., Roush T., Fong W., 1994, ApJ, 421, 615

Quillen A. C., Thorndike S., 2002, ApJ, 578, L149

Reffert S., Quirrenbach A., 2011, A\&A, 527, A140

Rhee J. et al., 2005, ApJ, 620, 1010

Rhee J., Song I., Zuckerman B., McElwain M., 2007, ApJ, 660, 1556

Ricarte A., Moldvai N., Hughes A. M., Duchêne G., Williams J. P., Andrews S. M., Wilner D. J., 2013, ApJ, 744, 80

Ricci L., Carpenter J. M., Fu B., Hughes A. M., Corder S., Isella A., 2015a, ApJ, 798, 124

Ricci L., Maddison S. T., Wilner D., MacGregor M. A., Ubach C., Carpenter J. M., Testi L., et al., 2015b, ApJ, 813, 138

Riviere-Marichalar P., 2013, A\&A, 555, A67

Roberge A. et al., 2013, ApJ, 771, 69

Roccatagliata V., Henning T., Wolf S., Rodmann J., Corder S., Carpenter J. M., Meyer M. R., Dowell D., 2009, ApJ, 497, 409

Rodriguez D. R., Zuckerman B., 2012, ApJ, 745, 147R 
Roseboom I. G. et al., 2013, MNRAS, 436, 430

Sadakane K., Nishida M., 1986, PASP, 98, 685

Schneider G. et al., 1999, ApJ, 513, L127

Schneider G. et al., 2006, ApJ, 650, 414

Schneider G., Weinberger A. J., Becklin E. E., Debes J. H., Smith B. A., 2009, AJ, 137, $53 \mathrm{~S}$

Schneider G. et al., 2014, AJ, 148, 59S

Schneider G. et al., 2016, AJ, 152, 64

Schüppler Ch. et al., 2015, A\&A, 581, A97

Schütz O., Nielbock M., Wolf S., Henning T., Els S., 2004, A\&A, 414, L9

Sheret I., Dent W. R. F., Wyatt M. C., 2004, MNRAS, 348, 1282

Sibthorpe B. et al., 2010, A\&A, 518, L130

Silverstone M. D., 2000, PhD thesis, UCLA

Söderhjelm S., 1980, A\&A, 89, 100

Song I., Caillault J.-P., Barrado y Navascués D., Stauffer J. R., 2001, ApJ, 546,352

Song I., Zuckerman B., Bessell M. S., 2004, ApJ, 614, 125 S

Soummer R. et al., 2014, ApJ, 786, L23

Stapelfeldt K., Krist J., Bryden G., Chen C., 2007, in Kalas P., ed, the Spirit of Bernard Lyot: the Direct Detection of Planets and Circumstellar Discs in the 21st Century, Univ. California, Berkeley, CA, p. 47

Stark C. C., Schneider G., Weinberger A. J., Debes J. H., Grady C. A. Jang-Condell H., Kuchner M. J., 2014, ApJ, 789, 58

Steele A., Hughes A. M., Carpenter J., Ricarte A., Andrews S. M., Wilner D. J., Chiang E., 2016, ApJ, 816, 27

Stencel R. E., Backman D. E., 1991, ApJS, 75, 905

Su K. et al., 2005, ApJ, 628, 487S

Su K. et al., 2006, ApJ, 653, 675

Su K., Rieke G. H., Stapelfeldt K. R., Smith P. S., Bryden G., Chen C. H., Trilling D. E., 2008, ApJ, 679, L125

Su K. et al., 2009, ApJ, 705, 314

Su K. et al., 2013, ApJ, 763, 118S

Sylvester R. J., Skinner C. J., Barlow M. J., Mannings V., 1996, MNRAS, 279, 915

Takeda G., Ford E. B., Sills A., Rasio F. A., Fischer D. A., Valenti J. A., 2007, ApJS, 168, 297

Tanner A., Beichman C., Bryden G., Lisse C., Lawler S., 2009, ApJ, 704, 109

Thebault P., Haghighipour N., 2015, in Jin S., Haghighipour N., Ip W.-H., eds, Planetary Exploration and Science: Recent Results and Advances. Springer Geophysics, Springer-Verlag, Berlin Heidelberg, p. 309

Thureau N. D. et al., 2014, MNRAS, 445, 2558

Torres G., Stefanik R. P., Latham D. W., Mazeh T., 1995, ApJ, 452, 870

Torres C. A. O., Quast G. R., Melo C. H. F., Sterzik M. F., 2008, in Reipurth B. , ed., Handbook of Star Forming Regions, Vol. II: The Southern Sky, ASP Monograph Publications, Vol. 5, p. 757

Trilling D. et al., 2007, ApJ, 658, 1289

Trilling D. et al., 2008, ApJ, 674, 1086

Tuomi M. et al., 2013, A\&A, 551, A79

van Leeuwen F. ed., 2007, Astrophysics and Space Science Library, Vol. 350, Hipparchos, the New Reduction of the Raw Data

Vican L., 2012, AJ, 143, 135

Vican L., Schneider A., Bryden G., Melis C., Zuckerman B., Rhee J., Song I., 2016, ApJ, 833, 263

Wahhaj Z., Koerner D. W., Sargent A. I., 2007, ApJ, 661, 368
Walker H. J., Butner H. M., 1995, Ap\&SS, 224, 389W

Walker H. J., Wolstencroft R. D., 1988, PASP, 100, 1509

Wenger M. et al., 2000, A\&AS, 143, 9

White J. A., Boley A. C., Dent W. R. F., Ford E. B., Corder S., 2017, MNRAS, 466, 4201

Williams J. P., Andrews S. M., 2006, ApJ, 653, 1480

Williams J. P. et al., 2004, ApJ, 604, 414

Wilner D. J., Holman M. J., Kuchner M., Ho P. T. P., 2002, ApJ, 569, L115

Wilner D. J., Andrews S. M., MacGregor M. A., Hughes A. M., 2012, ApJ, 749, L27

Wright E. L. et al., 2010, AJ, 140, 1868

Wyatt M. C., 2003, ApJ, 598, 1321

Wyatt M. C., 2006, ApJ, 639, 1153

Wyatt M. C., 2008, ARA\&A, 46, 339

Wyatt M. C., Dent W. R. F., 2002, MNRAS, 334, 589

Wyatt M. C., Dermott S. F., Telesco C. M., 2000, in Garzon F. et al., eds, ASP Conf. Ser., Vol. 219, Disks, Planetesimals and Planets, p. 289

Wyatt M. C., 2005, A\&A, 433, 1007

Wyatt M. C., Smith R., Su K. Y. L., Rieke G. H., Greaves J. S., Beichman C. A., Bryden G., 2007, ApJ, 663, 365

Wyatt M. C., Clarke C. J., Greaves J. S., 2007, MNRAS, 380, 1737

Wyatt M. C. et al., 2012, MNRAS, 424, 1206

Wyatt M. C., Panić O., Kennedy G. M., Matrà L., 2015, Ap\&SS, 357, 103

Zechmeister M. et al., 2011, A\&A, 552, A78

Zorec J., Royer F., 2012, A\&A, 537, A120

Zuckerman B., Becklin E. E., 1993, ApJ, 414, 793

Zuckerman B., Song I., 2004, ApJ, 603, 738

Zuckerman B., Song I., 2012, ApJ, 758, 77

Zuckerman B., Forveille T., Kastner J. H., 1995, Nature, 373, 494

Zuckerman B., Rhee J. H., Song I., Bessell M. S., 2011, ApJ, 732, 61

\section{APPENDIX A: THE SONS SURVEY IMAGES AND SPECTRAL ENERGY DISTRIBUTIONS}

This Appendix presents the $850 \mu \mathrm{m}$ S/N images from the SONS survey and SEDs together with model fits to the stellar photosphere and infrared to millimetre thermal (disc) excess. Unless otherwise stated, the colours are scaled from $-3 \sigma$ to the maximum $\mathrm{S} / \mathrm{N}$ in the images. Similarly the contours start at $-3 \sigma$ (dashed white) and then solid colours from $3 \sigma$ to the maximum in $1 \sigma$ steps. The white bar represents the spatial scale corresponding to the FWHM beam in astronomical units at the star. The 'star symbol' represents the position of the star with respect to the disc, taking into account any proper motion corrections if required.

For the SEDs, the black symbols represent measured fluxes whilst the brown symbols are stellar photosphere-subtracted values (i.e. disc fluxes from the infrared to millimetre excess). Small green dots are the star-subtracted Spitzer/IRS spectrum (if it exists). The grey and black inverted triangles represent the $3 \sigma$ upper flux limits (again, star-subtracted). Photometry from the SONS survey is highlighted by the blue circles. 

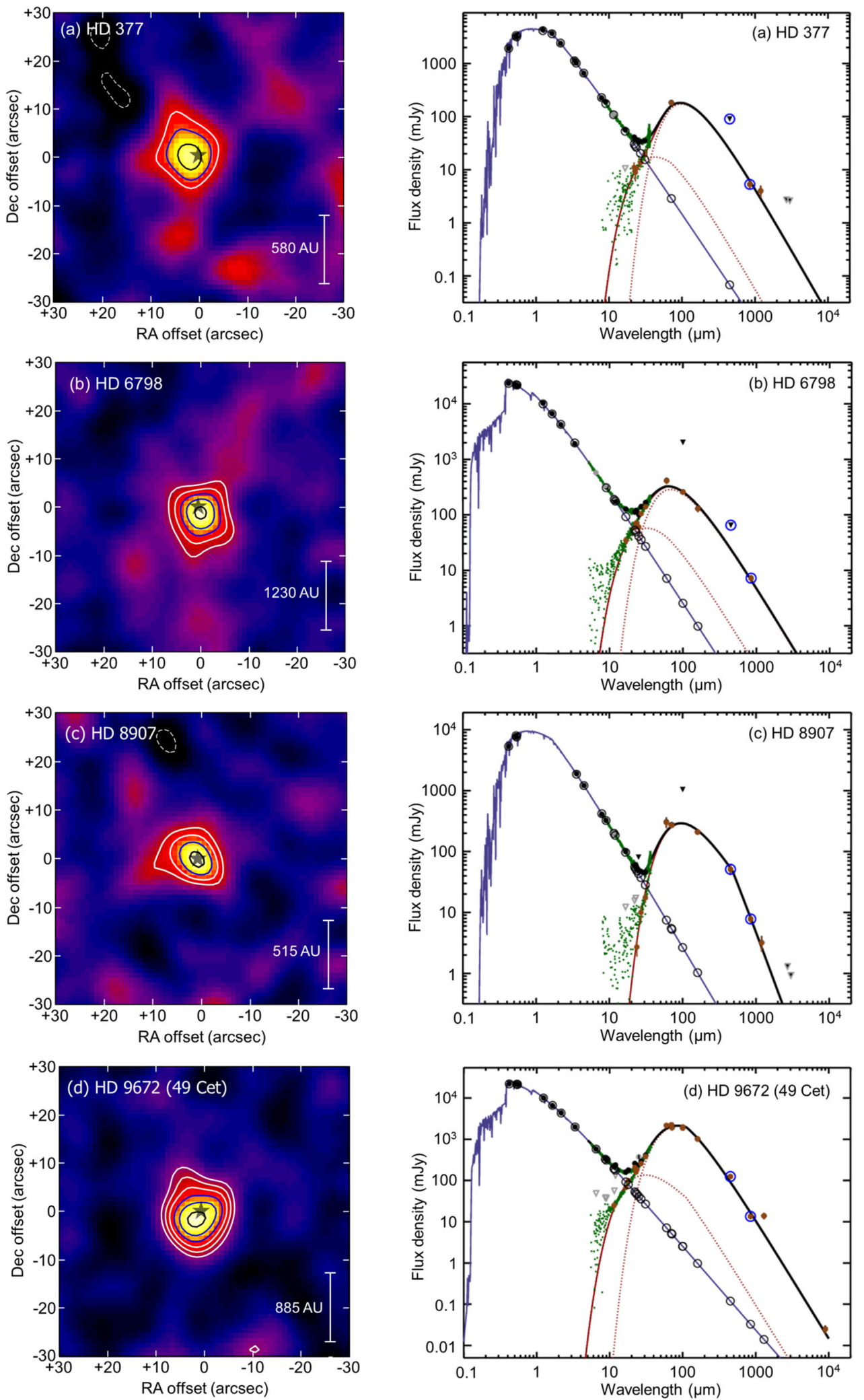

Figure A1. $850 \mu \mathrm{m} \mathrm{S/N}$ images and SEDs for the targets (a) HD 377, (b) HD 6798, (c) HD 8907 and (d) HD 9672 (49 Cet). 

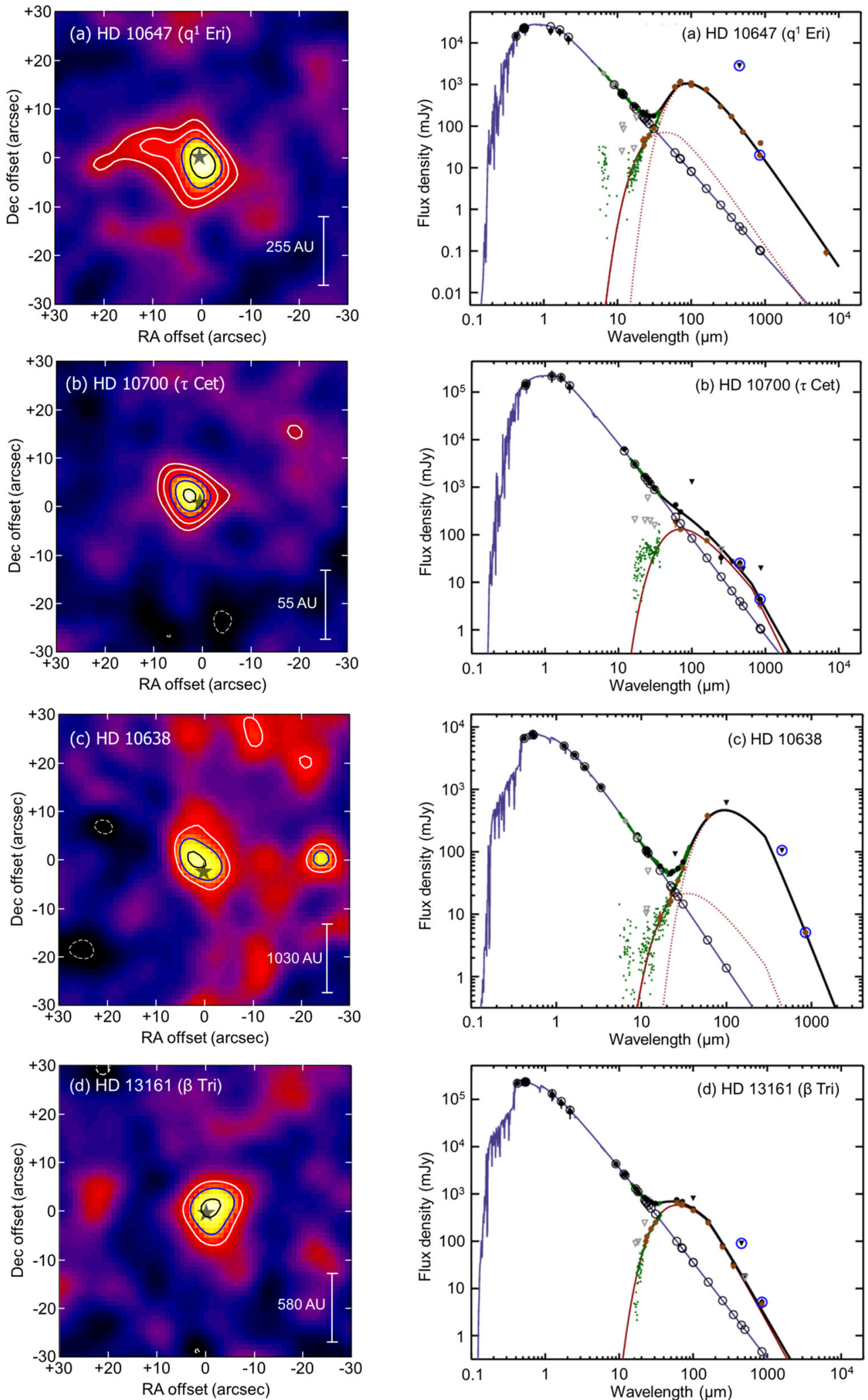

Figure A2. As for Fig. A1 for the targets (a) HD 10647 (q ${ }^{1}$ Eri), (b) HD 10700 ( $\tau$ Cet), (c) HD 10638 and (d) HD 13161 ( $\beta$ Tri). 

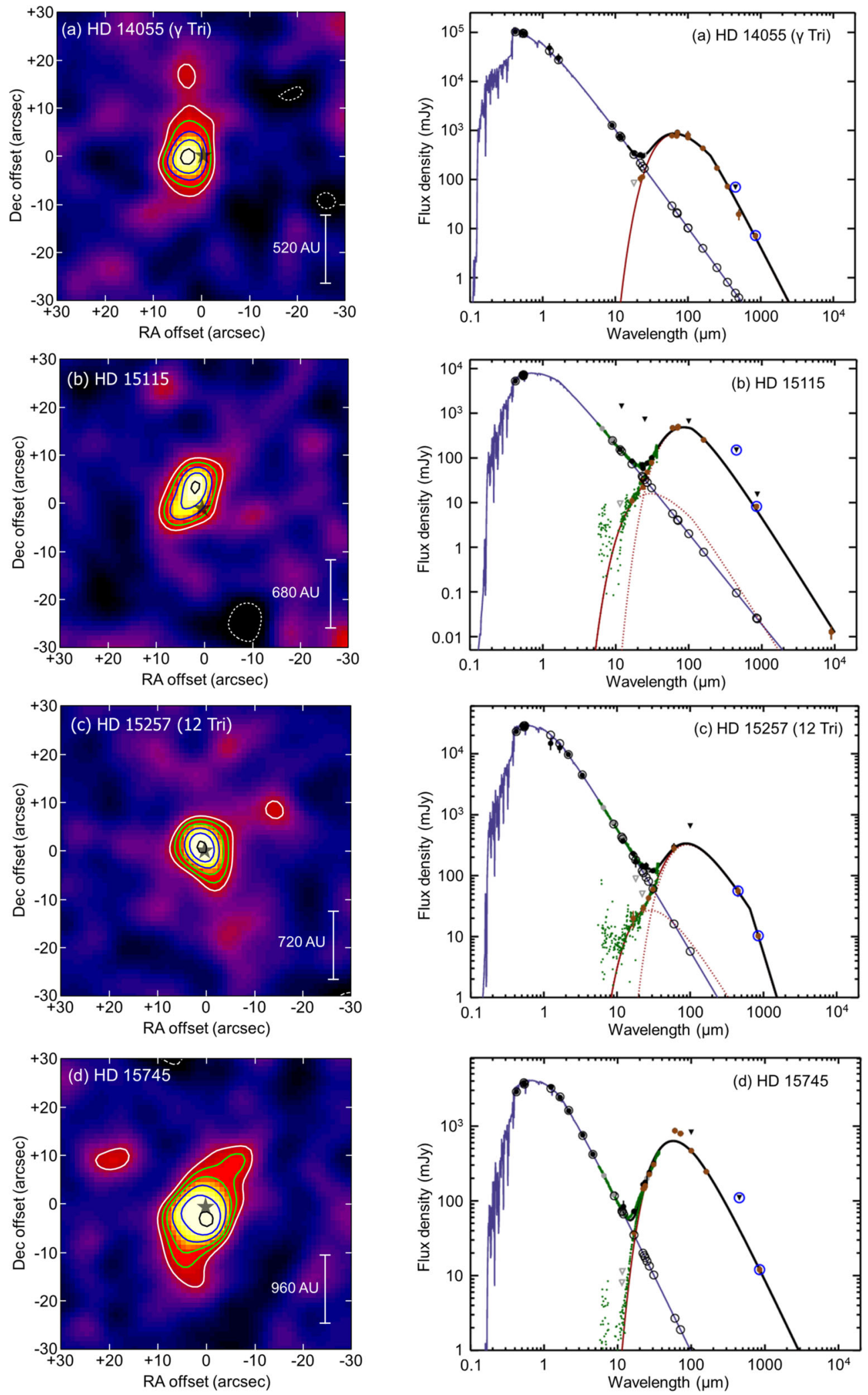

Figure A3. As for Fig. A1 for the targets (a) HD 14055 ( $\gamma$ Tri), (b) HD 15115, (c) HD 15257 (12 Tri) and (d) HD 15745. 

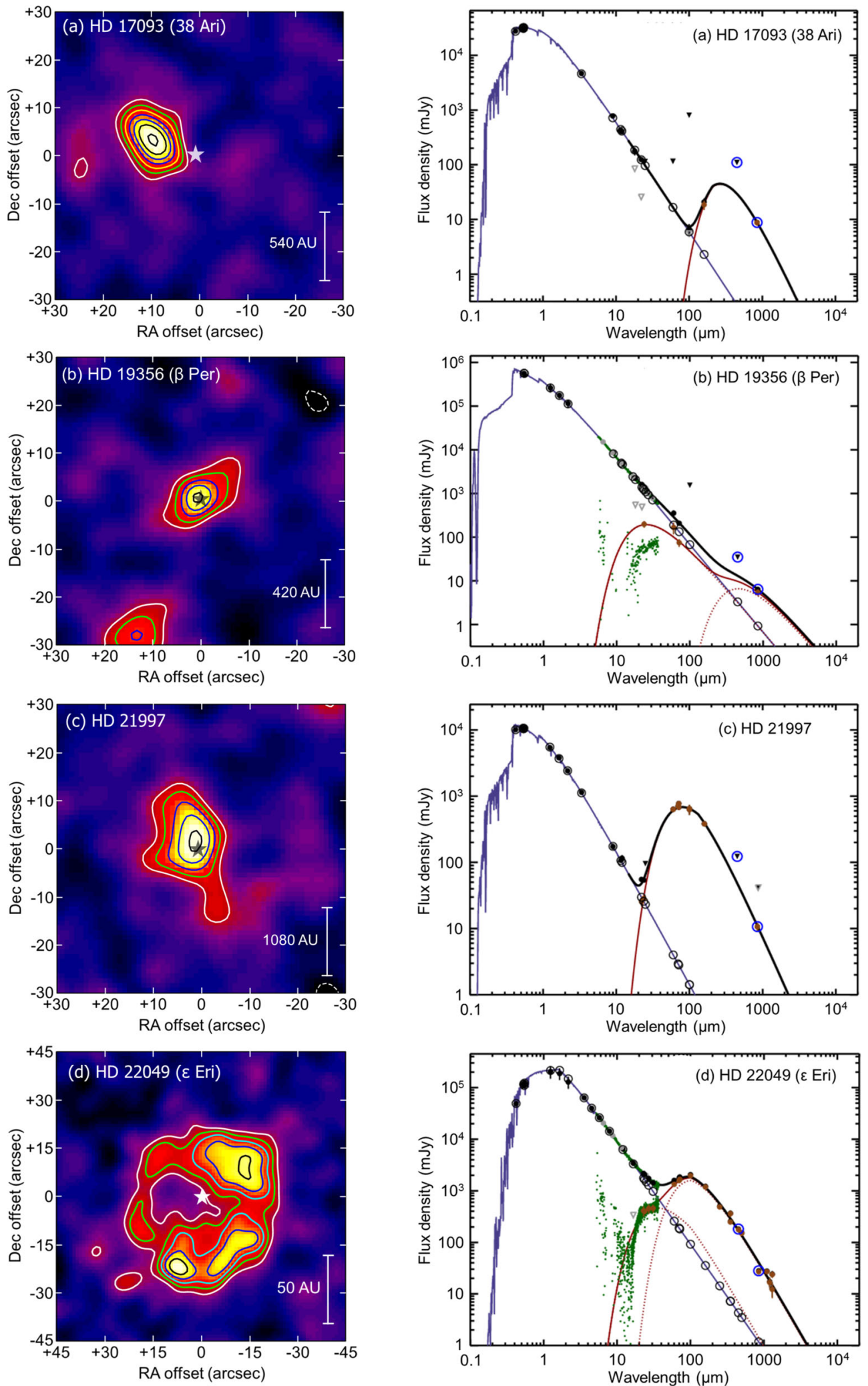

Figure A4. As for Fig. A1 for the targets (a) HD 17093 (38 Ari), (b) HD 19536 ( $\beta$ Per), (c) HD 21997 and (d) HD 22049 ( $\epsilon$ Eri). 

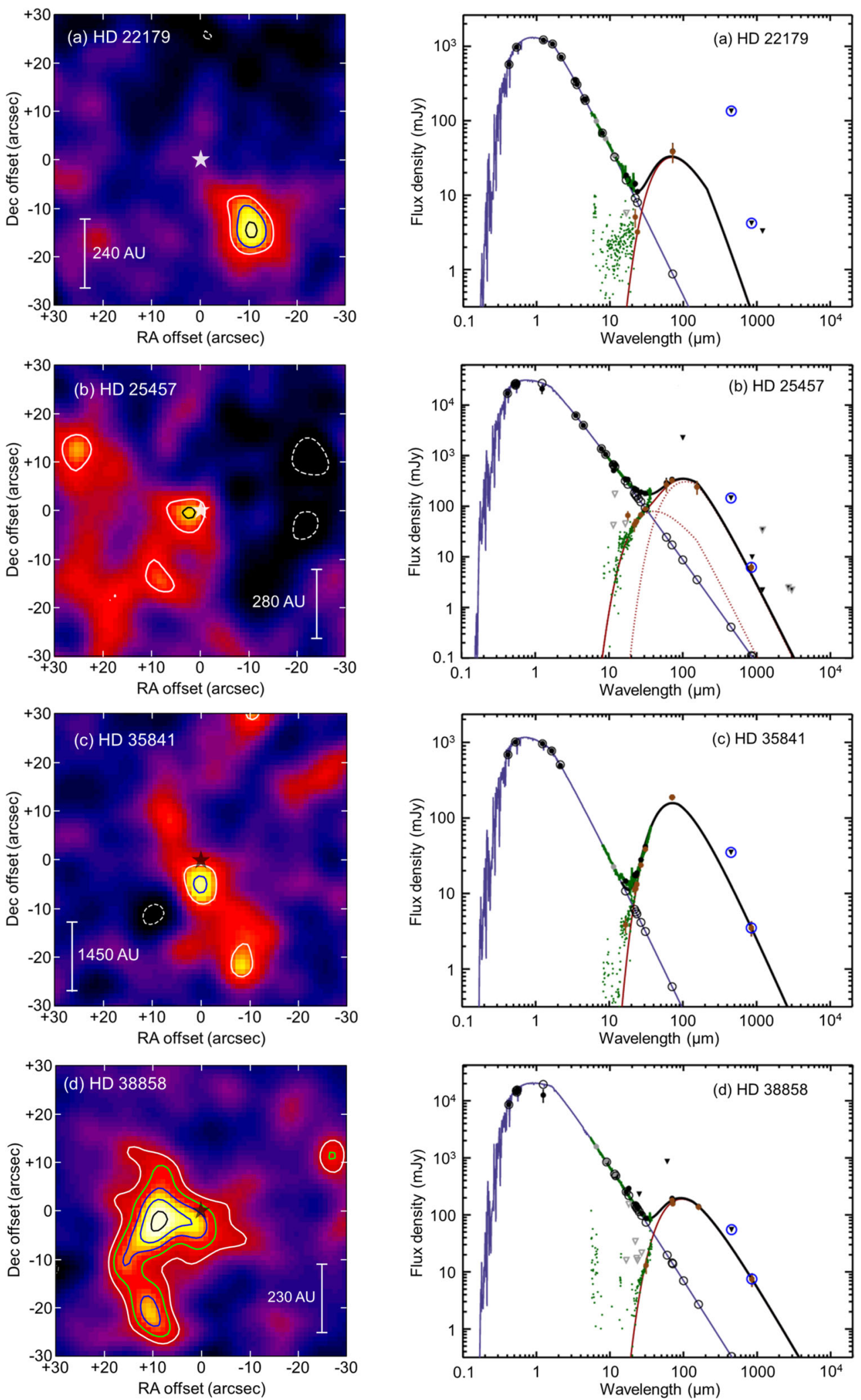

Figure A5. As for Fig. A1 for the targets (a) HD 22179, (b) HD 25457, (c) HD 35841 and (d) HD 38858. 

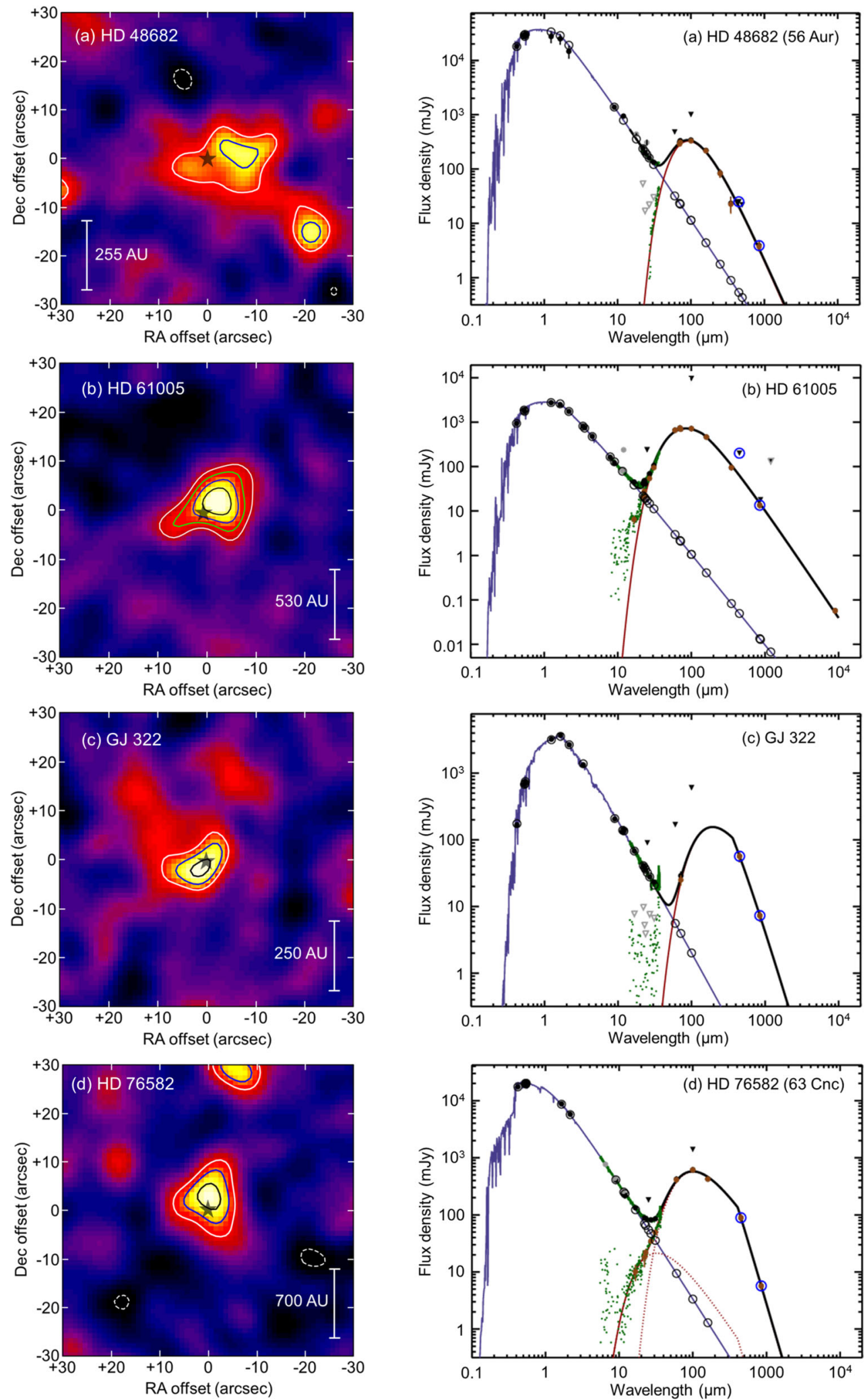

Figure A6. As for Fig. A1 for the targets (a) HD 48682, (b) HD 61005, (c) GJ 322 and (d) HD 76582 (63 Cnc). 

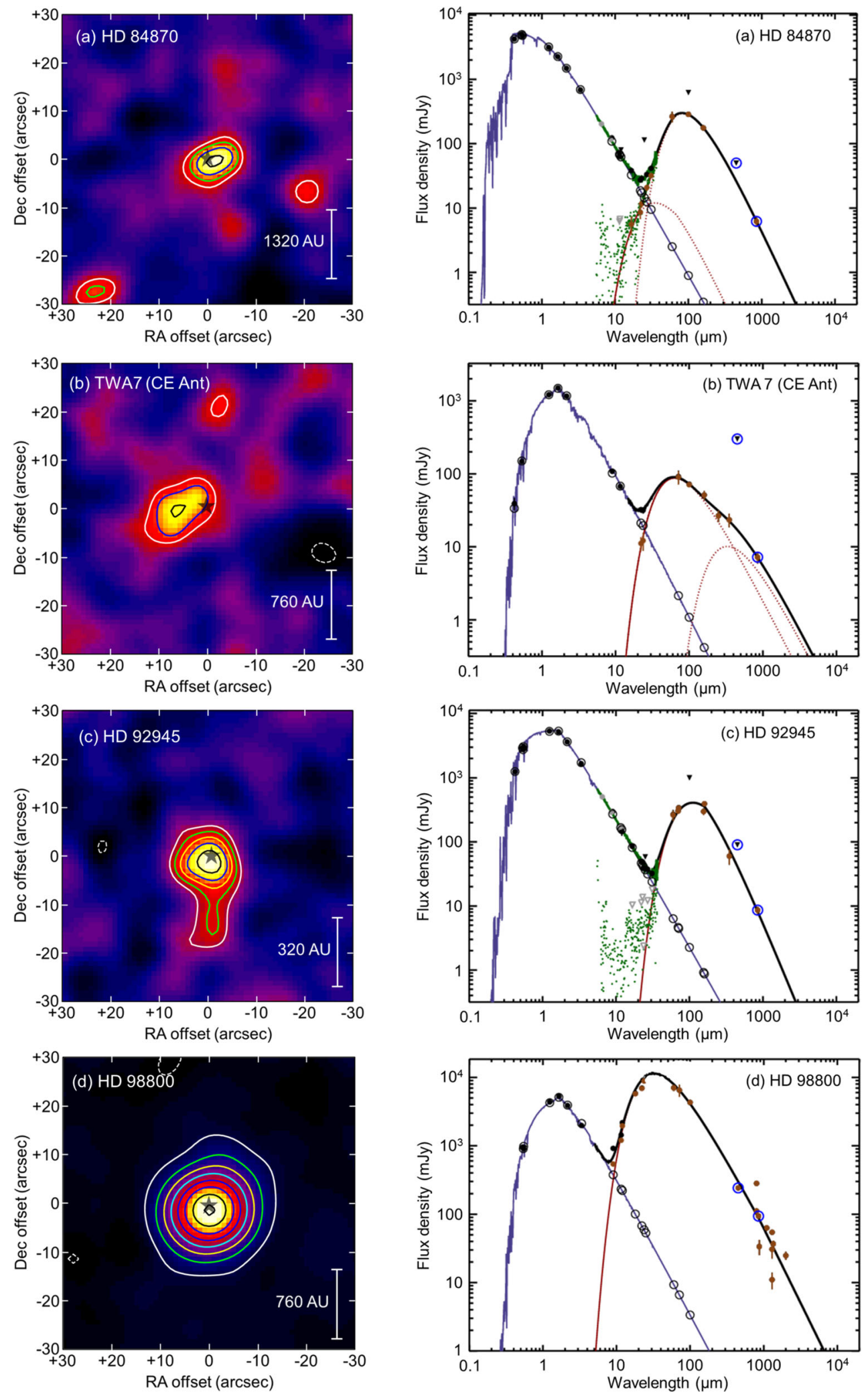

Figure A7. As for Fig. A1 for the targets (a) HD 84870, (b) TWA7 (CE Ant), (c) 92945 and (d) HD 98800 . The contours for HD 98800 are $-4 \sigma$ (dashed) and then solid contours starting at $4 \sigma$ and increasing in $8 \sigma$ steps. 

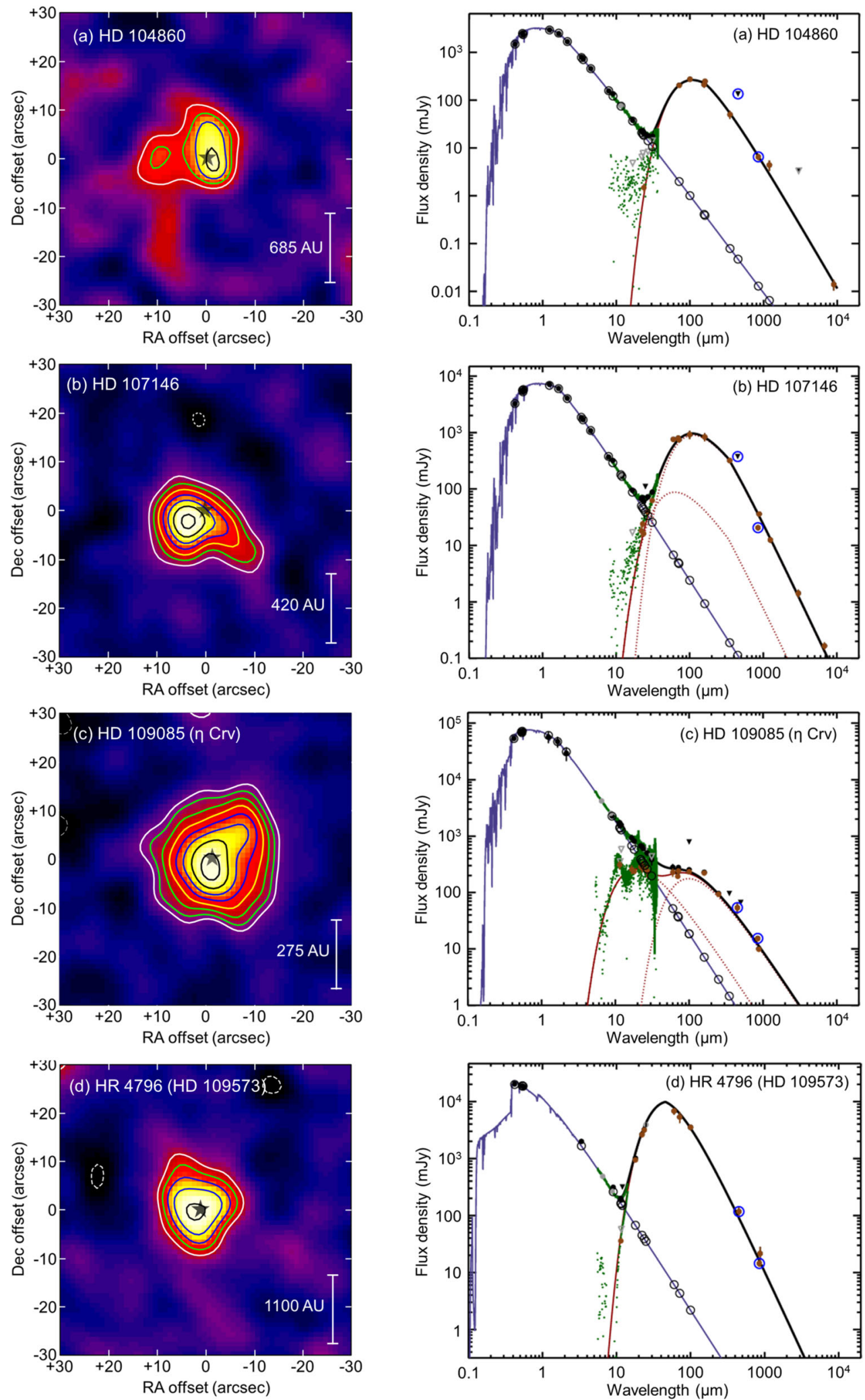

Figure A8. As for Fig. A1 for the targets (a) HD 104860, (b) HD 107146, (c) HD 109085 ( $\eta$ Crv) and (d) HR 4796 (HD 109573 ). 

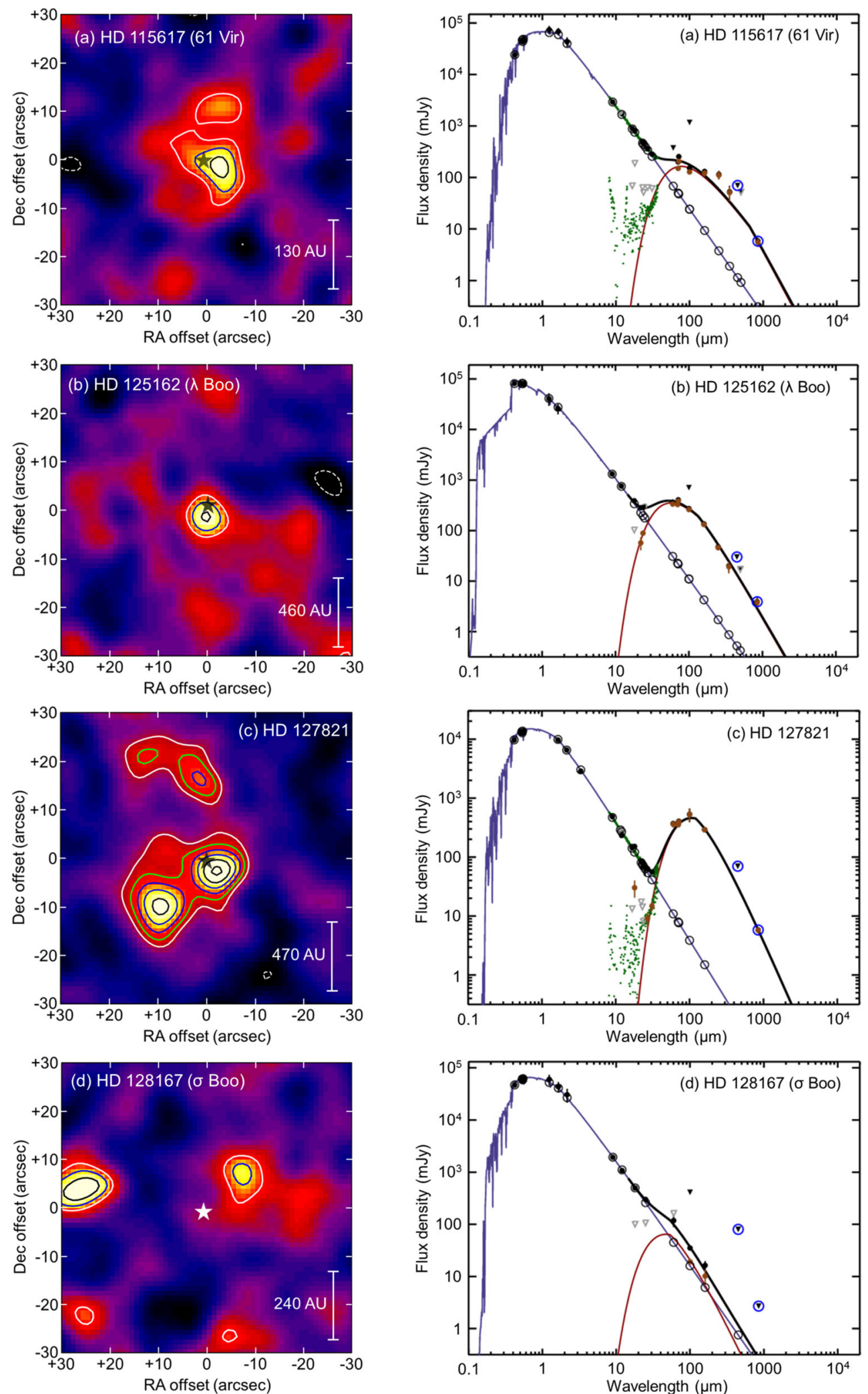

Figure A9. As for Fig. A1 for the targets (a) HD 115617 (61 Vir), (b) HD 125162 ( $\lambda$ Boo), (c) HD 127821 and (d) HD 128167 ( $\sigma$ Boo). 

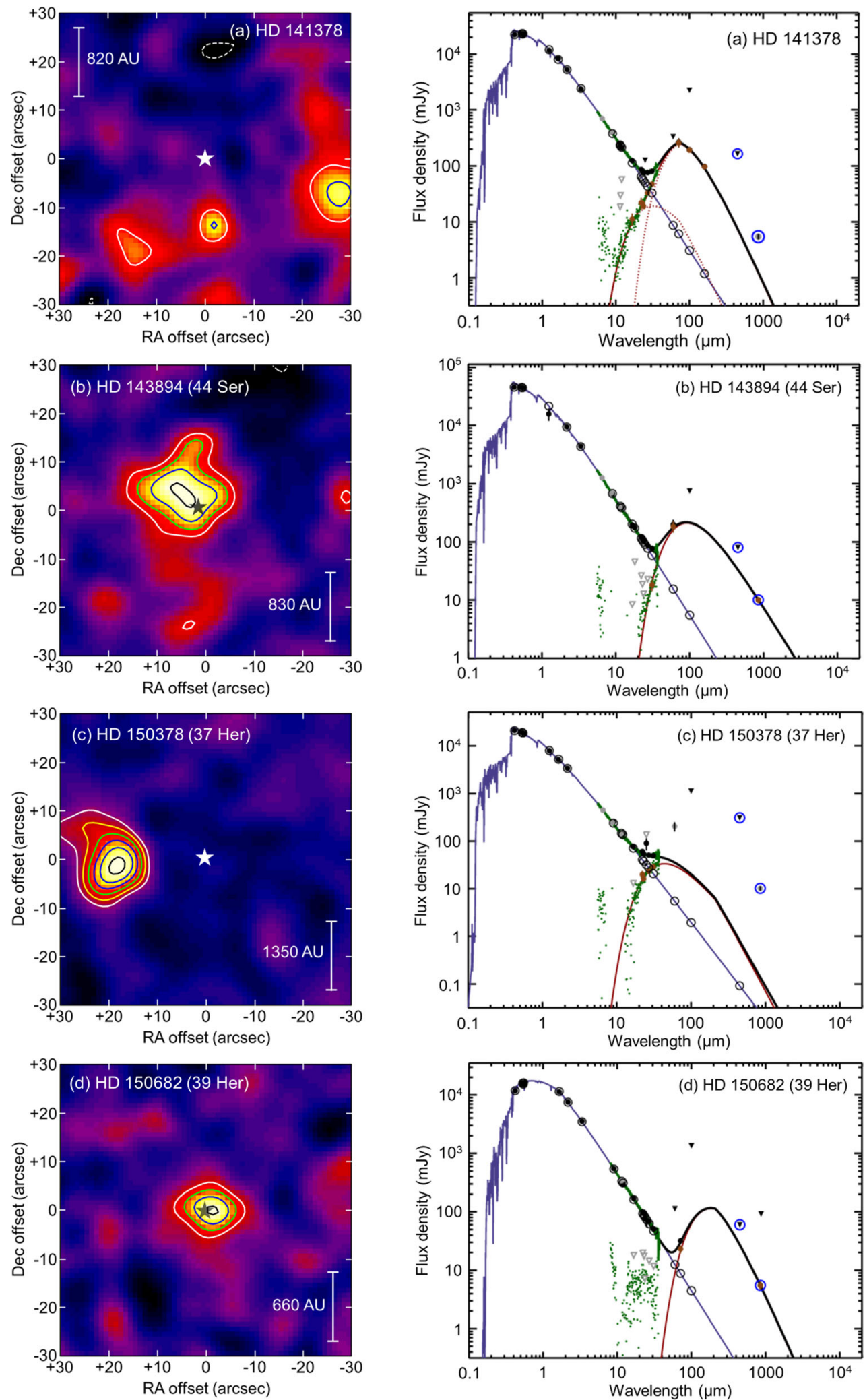

Figure A10. As for Fig. A1 for the targets (a) HD 141378, (b) HD 143894 (44 Ser), (c) HD 150378 (37 Her) and (d) HD 150682 (39 Her). 

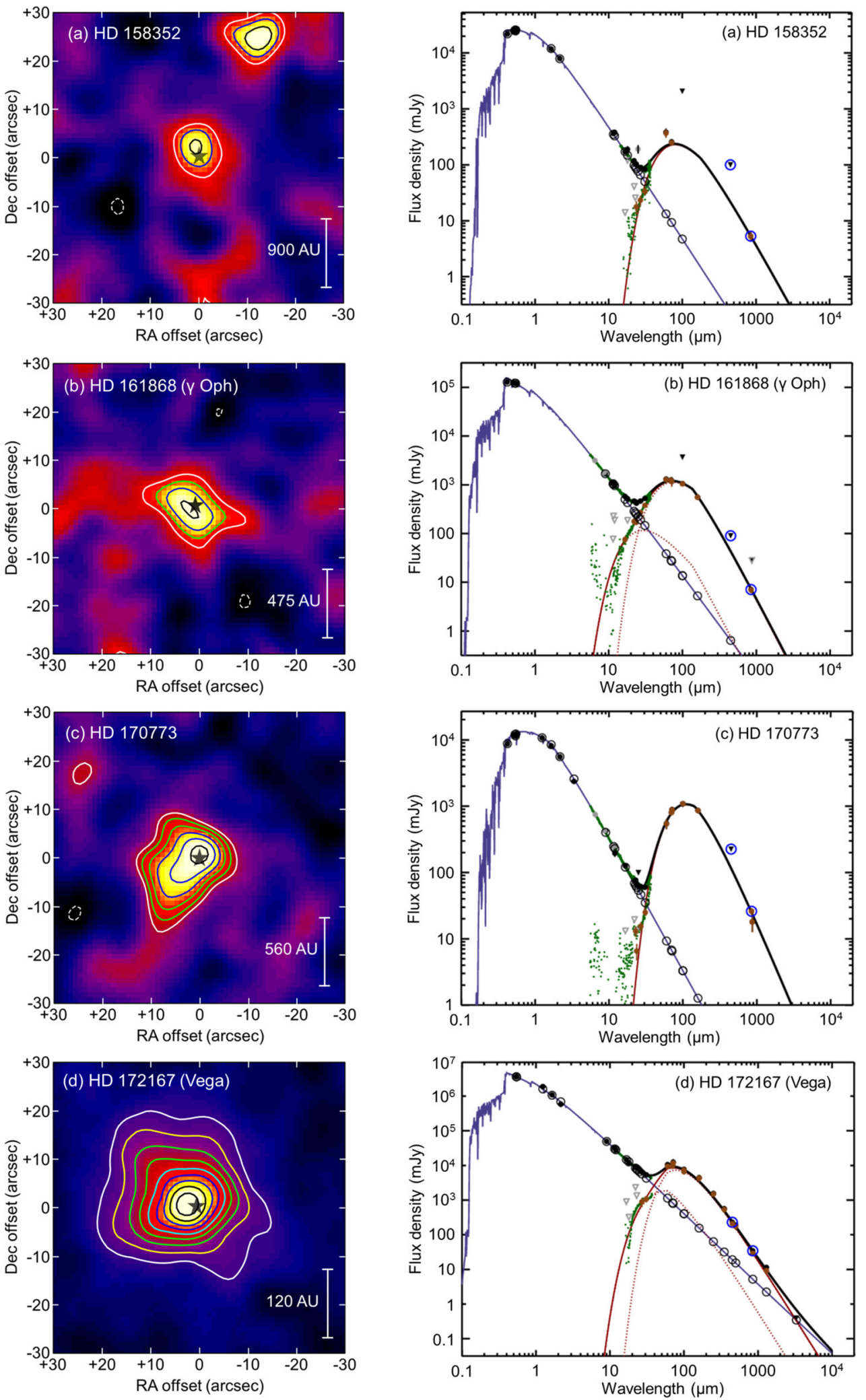

Figure A11. As for Fig. A1 for the targets (a) HD 158352, (b) HD 161868 ( $\gamma$ Oph), (c) HD 170773 and (d) HD 192167 (Vega). 

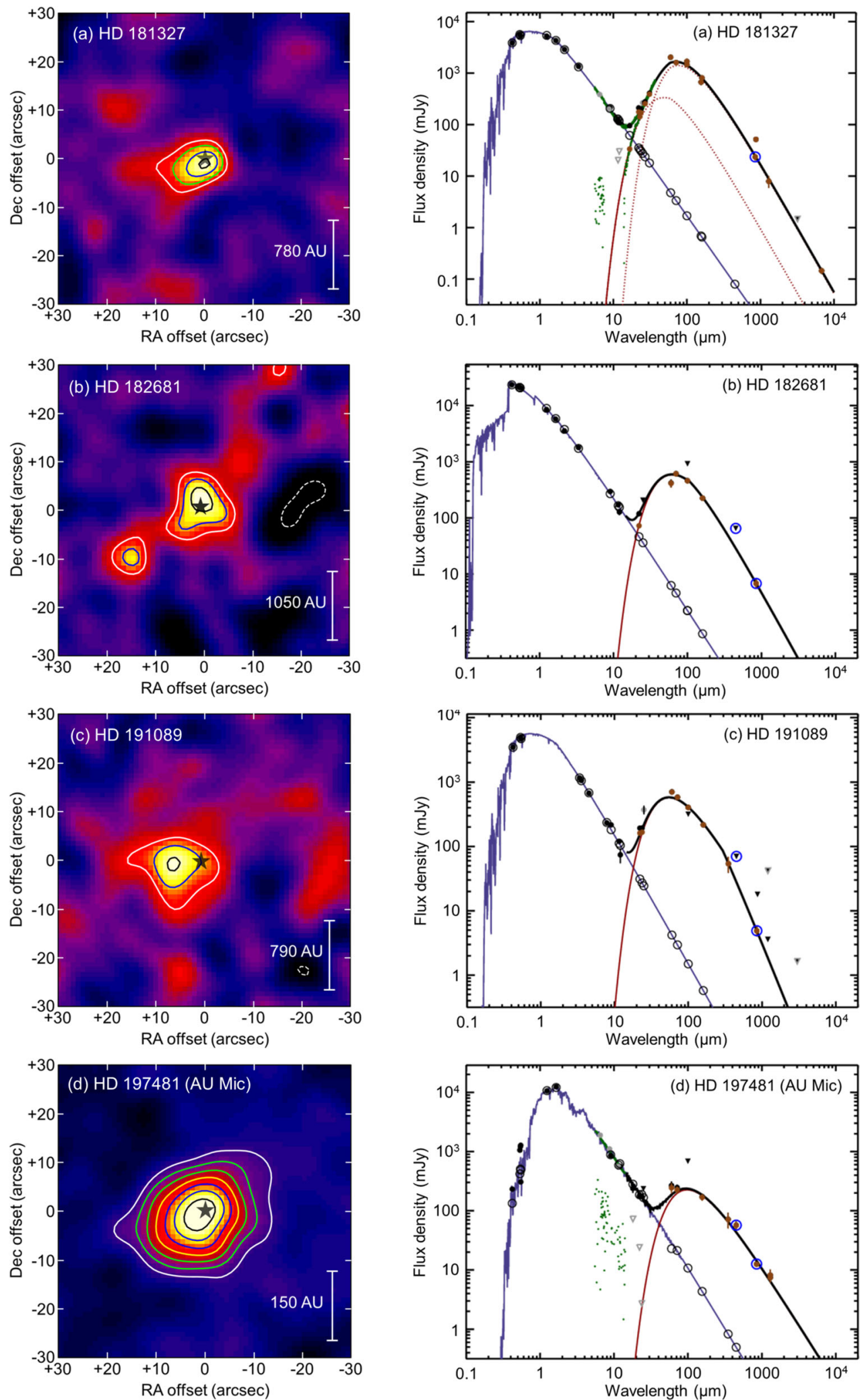

Figure A12. As for Fig. A1 for the targets (a) HD 181327, (b) HD 182681, (c) HD 191089 and (d) HD 197481 (AU Mic). The contours for the HD 197481 are $-3 \sigma$ (dashed) and then from $+4 \sigma$ in $2 \sigma$ steps. 

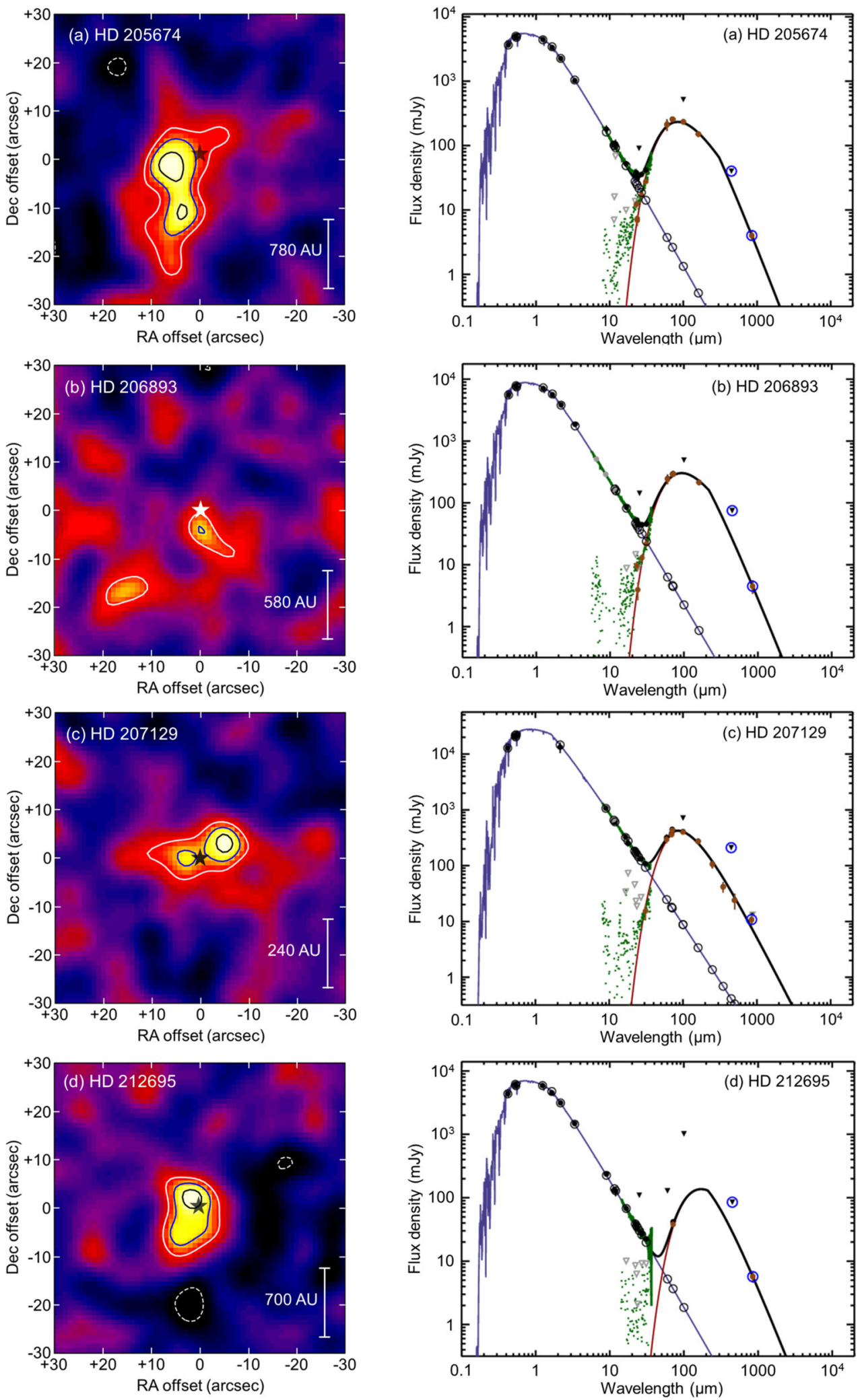

Figure A13. As for Fig. A1 for the targets (a) HD 205674, (b) HD 206893, (c) HD 207129 and (d) HD 212695. 

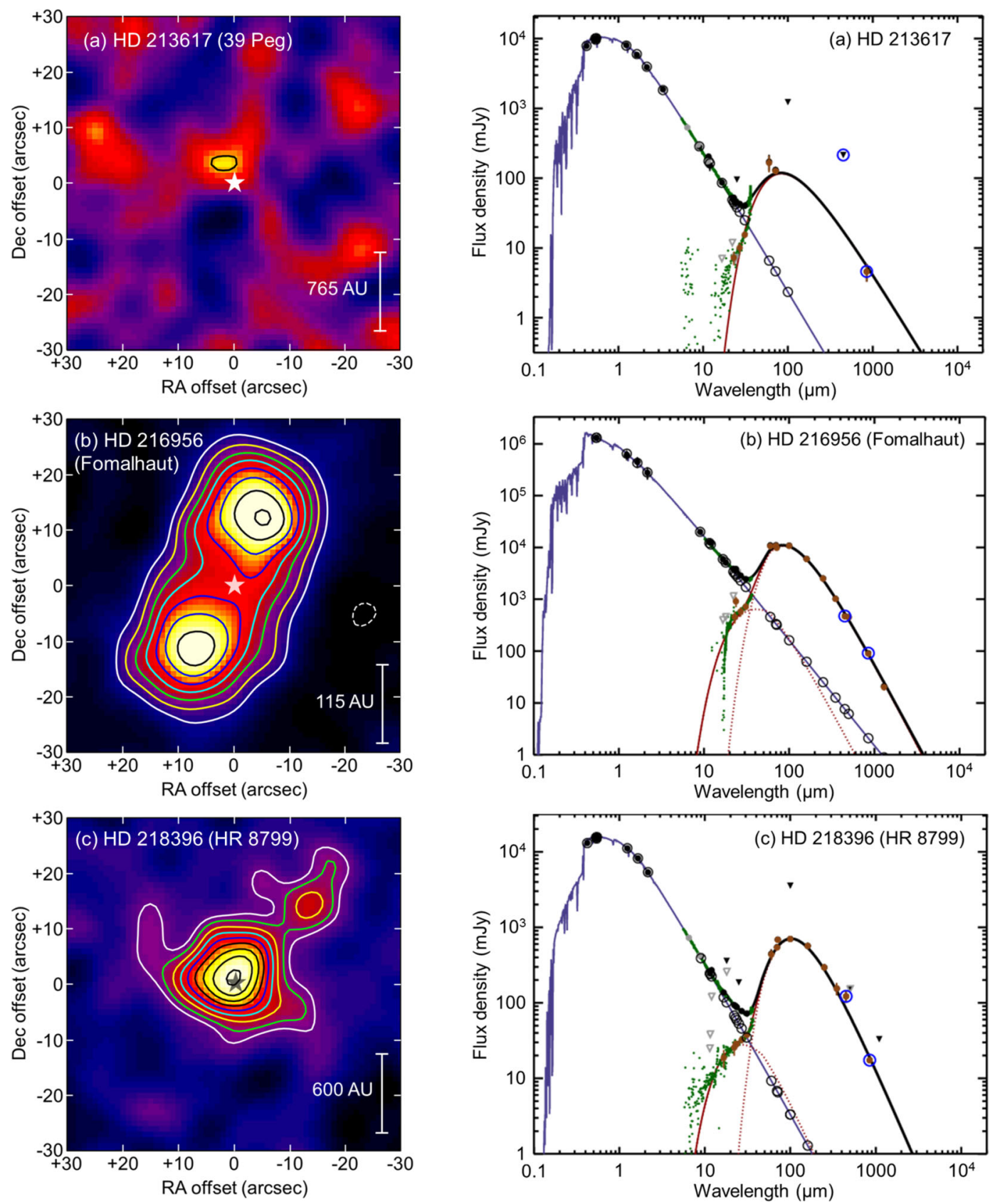

Figure A14. As for Fig. A1 for the targets (a) HD 213617, (b) HD 216956 (Fomalhaut) and (c) HD218396 (HR 8799 ). The contours for the HD 216396 are $-5 \sigma$ (dashed) and then from $+5 \sigma$ in $3 \sigma$ steps.

${ }^{1}$ UK Astronomy Technology Centre, Royal Observatory, Blackford Hill, Edinburgh, EH9 3HJ, UK

${ }^{2}$ Institute for Astronomy, University of Edinburgh, Royal Observatory, Blackford Hill, Edinburgh, EH9 3HJ, UK

${ }^{3}$ National Research Council of Canada Herzberg Astronomy \& Astrophysics Programs, 5071 West Saanich Road, Victoria, BC V9E 2E7, Canada

${ }^{4}$ Department of Physics \& Astronomy, University of Victoria, 3800 Finnerty Road, Victoria, BC V8P 5C2, Canada

${ }^{5}$ Institute of Astronomy, University of Cambridge, Madingley Road, Cambridge, CB3 OHA, UK

${ }^{6}$ School of Physics and Astronomy, University of St. Andrews, North Haugh, St. Andrews, Fife, KY16 9SS, UK

${ }^{7}$ Instituto de Astrofisica, Pontificia Universidad Catolica de Chile, Vicua Mackenna 4860, 7820436, Macul, Santiago, Chile
${ }^{8}$ Astrophysikalisches Institut and Universität-Sternwarte, FriedrichSchiller-Universität Jena, Schillergässchen 2-3, 07745 Jena, Germany

${ }^{9}$ Centre de recherche en astrophysique du Québec and Department de Physique, Université de Montreal, Montreal, QC H3C 3J7, Canada

${ }^{10}$ Jet Propulsion Laboratory, California Institute of Technology, 4800 Oak Grove Drive, Pasadena, CA 91109, USA

${ }^{11}$ Department of Physics and Astronomy, James Madison University, MSC 4502-901 Carrier Drive, Harrisonburg, VA 22807, USA

${ }^{12}$ Space Telescope Science Institute, 3700 San Martin Drive, Baltimore, MD 21218, USA

${ }^{13}$ Centre for Astrophysics Research, Science and Technology Research Institute, University of Hertfordshire, College Lane, Hatfield, Herts, AL10 9AB, $U K$

${ }^{14}$ Joint ALMA Observatory, Alonso de Cordova 3107, Vitacura 763-0355, Santiago, Chile 
${ }^{15}$ Astronomy Department, University of California, Berkeley, CA 94720-3411, USA

${ }^{16}$ Univ. Grenoble Alpes/CNRS, IPAG, F-38000 Grenoble, France

${ }^{17}$ Department of Physics \& Astronomy, University of British Columbia, 6224 Agricultural Road, Vancouver, BC V6T 1Z1, Canada

${ }^{18}$ Joint Astronomy Centre, 660 N. A'ohōkū Place, University Park, Hilo, HI 96720, USA

${ }^{19}$ European Southern Observatory, Karl-Schwarzschild-Str. 2, D-85738 Garching, Germany

${ }^{20}$ Observatoire de Paris, PSL Research University, CNRS, Sorbonne Universités, UPMC, 61 Av. de l'Observatoire, F-75014 Paris, France

${ }^{21}$ School of Physics, UNSW Australia, High Street, Kensington, NSW 2052, Australia

${ }^{22}$ Australian Centre for Astrobiology, UNSW Australia, High Street, Kensington, NSW 2052, Australia

${ }^{23}$ Computational Engineering and Science Research Centre, University of Southern Queensland, Toowoomba, QLD 4350, Australia

${ }^{24}$ Center for Astrophysical Sciences, Johns Hopkins University, Baltimore, MD 21218, USA
${ }^{25}$ School of Physical Sciences, The Open University, Milton Keynes, $M K 7$ 6AA, UK

${ }^{26}$ SRON Netherlands Institute for Space Research, NL-9747 AD Groningen, The Netherlands

${ }^{27}$ Department of Physics \& Astronomy, University of California, Los Angeles, 90095, USA

${ }^{28}$ Jeremiah Horrocks Institute, University of Central Lancashire, Preston, Lancashire, PRI 2HE, UK

${ }^{29}$ Leiden Observatory, Leiden University, PO Box 9512, NL-2300 RA Leiden, The Netherlands

${ }^{30}$ RAL Space, The Rutherford Appleton Laboratory, Chilton, Didcot, OX11 ONL, UK

${ }^{31}$ Harvard-Smithsonian Center for Astrophysics, 60 Garden Street, Cambridge, MA 02138, USA

This paper has been typeset from a $\mathrm{T}_{\mathrm{E}} \mathrm{X} / \mathrm{L} \mathrm{T} \mathrm{E} \mathrm{X}$ file prepared by the author. 